Resolubilidade global para uma classe de sistemas involutivos 



\section{Resolubilidade global para uma classe de sistemas involutivos ${ }^{1}$}

\section{Cleber de Medeira}

Orientador: Prof. Dr. Adalberto Panobianco Bergamasco

Coorientador: Prof. Dr. Sérgio Luís Zani

Tese apresentada ao Instituto de Ciências Matemáticas e de Computação - ICMC-USP, como parte dos requisitos para obtenção do título de Doutor em Ciências - Matemática. VERSÃO REVISADA.

USP - São Carlos

Maio de 2012

\footnotetext{
${ }^{1}$ Este trabalho teve suporte financeiro da FAPESP e da CAPES.
} 
Ficha catalográfica elaborada pela Biblioteca Prof. Achille Bassi e Seção Técnica de Informática, ICMC/USP, com os dados fornecidos pelo(a) autor(a)

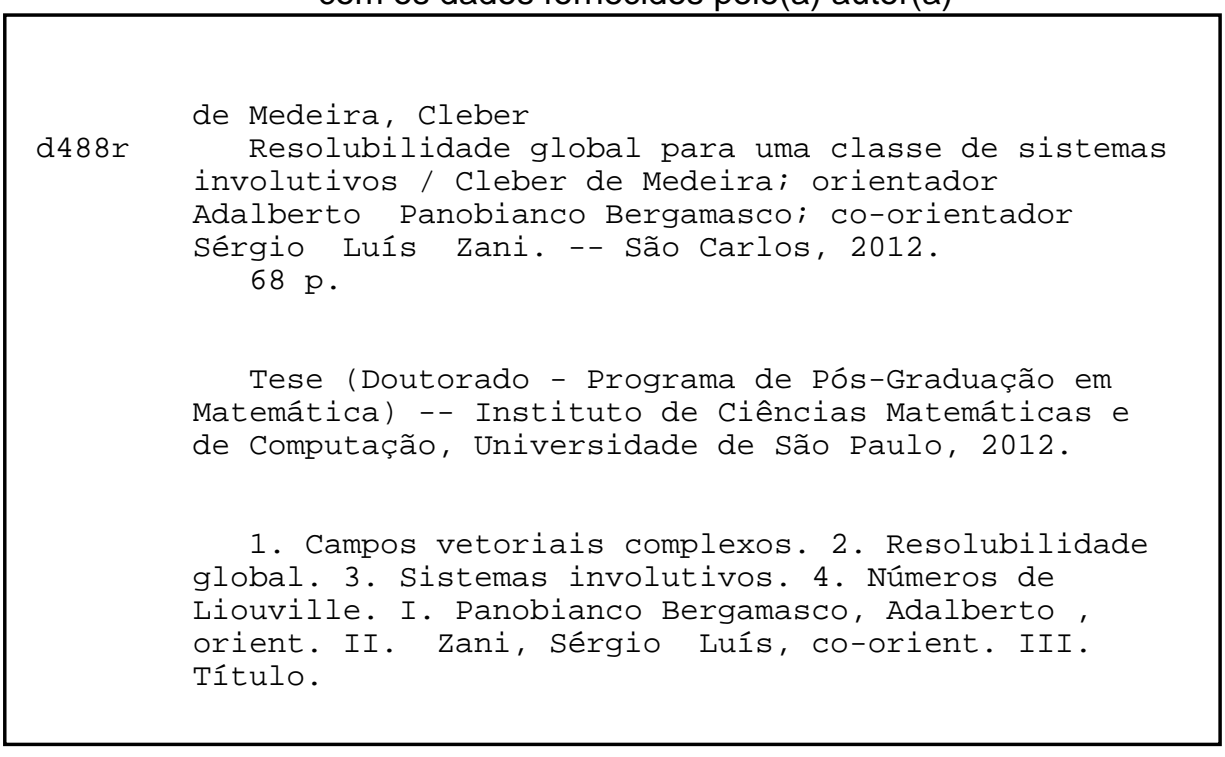


Aos meus pais

João e Teresinha; e à minha esposa Ana Eliza. 



\section{Agradecimentos}

Agradeço primeiramente a Deus, pela vida e por despertar em mim o gosto pela Matemática.

Sou grato aos meus pais Teresinha e João de Medeira, por minha formação como pessoa e pelo constante incentivo na vida estudantil;

À minha esposa Ana Eliza que tem sido minha grande companheira nesses árduos anos de estudo e distância, sempre com muita compreensão e carinho;

Aos meus irmãos Cleverson e Gabriel de Medeira, pelo apoio recebido em todos os momentos importantes da minha vida;

Aos meus orientadores do doutorado, Prof. Adalberto e Prof. Zani, pela confiança depositada em mim, pela paciência e dedicação durante estes últimos anos;

Ao meu orientador da iniciação científica, prof. Luis Grados (UEPG), e ao meu orientador do mestrado, prof. Higidio Oquendo (UFPR), pelo incentivo para a carreira acadêmica;

Aos professores e funcionários do ICMC, em especial o grupo de EDP's lineares, professores Adalberto Bergamasco, Sérgio Zani, Paulo Dattori, Wagner Nunes, Evandro da Silva e Alexandre Kirilov (UFPR) e aos colegas Giuliano, Rafael, Wanderley e Renato;

A todos os colegas de pós-graduação, em especial: André Furtado, Suzete Afonso, Marcos Pimenta, Éder Ritis, Márcio Jorge, José Claudinei, Thiago Catalan, John Beiro, Ligia, Vinicius e Alisson;

Aos amigos da "vila do Chaves", Josuel Kruppa, Luis Felipe e Lidiane;

Finalmente agradeço à FAPESP pelo suporte financeiro, fundamental para a realização deste trabalho. 

Estudamos a resolubilidade global de uma classe de sistemas involutivos com $n$ campos vetoriais suaves definidos no toro de dimensão $n+1$. Obtemos uma caracterização completa para o caso desacoplado desta classe em termos de formas de Liouville e da conexidade de todos os subníveis e superníveis, no espaço de recobrimento minimal, de uma primitiva global da 1-forma associada ao sistema. Além disso, apresentamos uma situação especial na qual o sistema não é globalmente resolúvel e usamos isso para obter alguns resultados em um caso com acoplamento mais forte. 

We study the global solvability of a class of involutive systems with $n$ smooth vector fields on the torus of dimension $n+1$. We obtain a complete characterization for the uncoupled case of this class in terms of Liouville forms and of the connectedness of all sublevel and superlevel sets of the primitive of a certain 1-form in the minimal covering space. Also, we exhibit a special situation where the system is not globally solvable and we use this to obtain some results in a more general case. 

Introdução $\quad$ xi

1 Considerações iniciais 1

1.1 O sistema de equações . . . . . . . . . . . . . . . . . 1

1.2 Condições de compatibilidade .................... 2

1.3 Recobrimento minimal de $\mathbb{T}^{n} \ldots \ldots \ldots \ldots$

1.4 Números de Liouville . . . . . . . . . . . . . . . . . . 9

2 Sistemas involutivos desacoplados $\quad \mathbf{1 3}$

2.1 Proposição 2.3: demonstração da suficiência . . . . . . . . . . . . 17

2.1.1 Condição suficiente $(i) \ldots \ldots \ldots \ldots 17$

2.1.2 Condição suficiente $(i i) \ldots \ldots . \ldots 22$

2.2 Proposição 2.3: demonstração da necessidade . . . . . . . . . . . 22

2.3 Proposição 2.4: demonstração da suficiência . . . . . . . . . . . . 33

2.3.1 Condição suficiente $(i i) \ldots \ldots \ldots$. . . . . . . . . 34

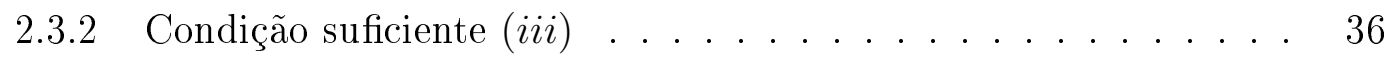

2.4 Proposição 2.4: demonstração da necessidade . . . . . . . . . . . . 37

2.4.1 Caso 1: $a_{0}^{\prime} \notin \mathbb{Q}^{m}$ é Liouville. . . . . . . . . . . . . 37

2.4.2 Caso 2: $a_{0}^{\prime} \in \mathbb{Q}^{m} \ldots \ldots \ldots \ldots$

3 Resultados adicionais $\quad 49$

3.1 Um teorema de não resolubilidade . . . . . . . . . . . . . . . . 50

3.2 Um resultado sobre subníveis e superníveis . . . . . . . . . . . . . 56

3.3 Sistema parcialmente acoplado - um caso . . . . . . . . . . . . 60 


\section{Referências Bibliográficas}

Índice Remissivo 
Nosso interesse neste trabalho é estudar a resolubilidade global de sistemas de campos vetoriais complexos do tipo

$$
L_{j}=\frac{\partial}{\partial t_{j}}+c_{j}(t) \frac{\partial}{\partial x}, \quad j=1, \ldots, n
$$

definidos no toro $\mathbb{T}^{n+1} \simeq(\mathbb{R} / 2 \pi \mathbb{Z})^{n+1}$, sendo $c_{j} \in C^{\infty}\left(\mathbb{T}^{n} ; \mathbb{C}\right)$ para $j=1, \ldots, n$ e $(t, x)=\left(t_{1}, \ldots, t_{n}, x\right)$ as coordenadas em $\mathbb{T}^{n+1}$. O sistema (1) é do tipo tubo (veja [BCH] página 24) e neste trabalho vamos supor que seja involutivo o que equivale à 1-forma $c(t)=\sum_{j=1}^{n} c_{j}(t) d t_{j}$ ser fechada.

O estudo da resolubilidade global do sistema (1) consiste em obter condições necessárias e/ou suficientes para que, dadas funções $f_{j} \in C^{\infty}\left(\mathbb{T}^{n+1}\right), j=1, \ldots, n$, satisfazendo certas condições naturais de compatibilidade, exista solução $u$ das equações diferenciais parciais

$$
L_{j} u=f_{j}, \quad j=1, \ldots, n .
$$

Não é conhecida uma resposta no caso geral para esse problema. Alguns trabalhos nos indicam condições para a resolubilidade do sistema (1) a partir de certas hipóteses. Pelo memorável trabalho [T1] de François Treves, a resolubilidade local do sistema (1) está relacionada com a conexidade de todos os conjuntos de subnível e supernível da parte imaginária de uma primitiva local da 1-forma $c$. Propriedades dessa natureza aparecem pela primeira vez nesse trabalho que trata de operadores em um contexto mais geral e em todos os níveis do complexo associado.

Pelo trabalho $[\mathrm{CH}]$ de Cardoso e Hounie, posterior a [T1], quando a 1-forma $c$ for exata, o sistema (1) será globalmente resolúvel se, e somente se, a parte imaginária de uma primitiva global de $c$ possuir todos os subníveis e superníveis conexos em $\mathbb{T}^{n}$. 
Quando a 1-forma $c$ não é exata, ela não possui uma primitiva global definida em $\mathbb{T}^{n}$, o que exige nova abordagem.

O trabalho $[\mathrm{H} \mid$ de Hounie apresenta uma resposta completa para a resolubilidade global quando o sistema (1) é composto por um único campo, isto é,

$$
L=\frac{\partial}{\partial t}+c(t) \frac{\partial}{\partial x}
$$

definido em $\mathbb{T}^{2}$. Neste caso, a resolubilidade global envolve também condições sobre a parte real $\operatorname{Re}(c)$. Mais precisamente, se $c_{0}$ é a média da função $c$ então o campo $L$ é globalmente resolúvel em $\mathbb{T}^{2}$ se, e somente se, vale alguma das seguintes condições:

1. Se $\operatorname{Im}(c) \equiv 0$ então $c_{0}$ não é um número de Liouville;

2. Se $\operatorname{Im}(c) \not \equiv 0$ e $c_{0} \in \mathbb{R}$ então uma primitiva de $\operatorname{Im}(c)$ possui apenas subníveis e superníveis conexos em $\mathbb{T}^{1}$ e $c_{0} \in \mathbb{Z}$;

3. Se $\operatorname{Im}(c) \not \equiv 0$ e $c_{0} \notin \mathbb{R}$ então $\operatorname{Im}(c)$ não muda de sinal.

Bergamasco e Kirilov estudaram em $\mid \mathrm{BK}\rfloor$ a resolubilidade global do sistema (1) considerando dois campos em $\mathbb{T}^{3}$ e $c=i b$, sendo $b=b_{1} d t_{1}+b_{2} d t_{2}$ uma 1-forma real com períodos $b_{10}$ e $b_{20}$ racionalmente independentes. Neste caso, a 1-forma $b$ não possui uma primitiva global definida em $\mathbb{T}^{2}$, então o que se utiliza é o pull-back de $b$ por meio do recobrimento universal $\Pi: \mathbb{R}^{2} \rightarrow \mathbb{T}^{2}$. Desse modo, o sistema (1) é globalmente resolúvel se, e somente se, todos os conjuntos de subnível e supernível de uma primitiva global do pull-back $\Pi^{*} b$ são conexos em $\mathbb{R}^{2}$.

O caso em que $c=i b$ sendo $b=b_{1} d t_{1}+b_{2} d t_{2}$ uma 1-forma real com períodos $b_{10}$ e $b_{20}$ racionalmente dependentes (não ambos nulos) foi estudado por Bergamasco, Kirilov, Zani e Nunes em [BKNZ]. Os autores mostraram que para esse caso a resolubilidade global é equivalente à conexidade de todos os subníveis e superníveis de uma primitiva global do pull-back de $b$ em $\mathbb{R} \times \mathbb{T}^{1}$.

Portanto, os trabalhos $[\mathrm{CH}]$, $|\mathrm{BKNZ}|$ e $[\mathrm{BK} \mid$, respectivamente, mostram que para $n=2$ e $c=i b$ a resolubilidade global do sistema (1) é equivalente à conexidade de todos os subníveis e superníveis de uma primitiva global do pull-back de $b$ no chamado espaço de recobrimento minimal de $\mathbb{T}^{2}$ com relação à 1-forma $b$. O recobrimento minimal é isomorfo a $\mathbb{R}^{r} \times \mathbb{T}^{2-r}$, onde $r=0,1,2$ depende dos períodos $b_{10}$ e $b_{20}$. Isso nos dá uma ideia da complexidade desse problema mesmo em dimensão $n=2$.

Outros artigos que tratam de questões semelhantes são [BCM], [BCP1], [BCP2], [BNZ1], |BNZ2] e [BZ]. 
No primeiro capítulo do trabalho estabelecemos as notações e resultados preliminares que são importantes para a compreensão do texto. Apresentamos o sistema de campos vetoriais complexos (1) inserido na teoria das estruturas localmente integráveis. Em seguida, definimos o significado de resolubilidade global para este trabalho. Além disso, definimos e expomos alguns resultados sobre números de Liouville, porém em vez de números reais são considerados vetores do $\mathbb{R}^{m}$ (ver Definição 1.5).

O Capítulo 2 trata da resolubilidade global do sistema (1) no caso $n$-dimensional assumindo que a parte imaginária de cada função $c_{j}$ depende apenas da variável $t_{j}$ correspondente, ou seja, $c_{j}(t)=a_{j}(t)+i b_{j}\left(t_{j}\right), j=1, \ldots, n$. Após uma certa conjugação mostramos que, para o estudo da resolubilidade global, basta considerar cada função $a_{j}$ constante, isto é,

$$
L_{j}=\frac{\partial}{\partial t_{j}}+\left(a_{j 0}+i b_{j}\left(t_{j}\right)\right) \frac{\partial}{\partial x}, \quad j=1, \ldots, n,
$$

com $a_{j 0}$ a média da função $a_{j}$. Para esse sistema damos o nome de sistema desacoplado.

Conseguimos condições necessárias e suficientes para que o sistema desacoplado seja globalmente resolúvel (ver Teorema 2.1). Essas condições novamente envolvem a propriedade de conexidade dos subníveis e superníveis de uma primitiva global do pull-back de $b=\operatorname{Im}(c)$ no recobrimento minimal de $\mathbb{T}^{n}$ com relação à 1 -forma $b$. Como consequência dos resultados obtidos temos que o sistema

$$
\left\{\begin{array}{l}
L_{1}=\frac{\partial}{\partial t_{1}}+\frac{1}{4} \frac{\partial}{\partial x} \\
L_{2}=\frac{\partial}{\partial t_{2}}+\left(\frac{1}{2}+i \operatorname{sen} t_{2}\right) \frac{\partial}{\partial x}
\end{array}\right.
$$

é globalmente resolúvel em $\mathbb{T}^{3}$, enquanto que

$$
\left\{\begin{array}{l}
L_{1}=\frac{\partial}{\partial t_{1}}+\frac{1}{2} \frac{\partial}{\partial x} \\
L_{2}=\frac{\partial}{\partial t_{2}}+\left(\frac{1}{4}+i \operatorname{sen} t_{2}\right) \frac{\partial}{\partial x}
\end{array}\right.
$$

não é globalmente resolúvel (ver Proposição 2.4).

Por fim, no Capítulo 3 apresentamos uma situação de não resolubilidade do sistema (1) quando $c=i b$, sendo $b$ uma 1-forma real, fechada e suave definida em $\mathbb{T}^{n}$ satisfazendo hipóteses adicionais. Como aplicação disso e usando resultados do trabalho [BK! obtemos condições necessárias e suficientes para a resolubilidade global em um caso parcialmente acoplado. 



\begin{tabular}{c|c|}
\hline \multirow{2}{*}{} & CAPÍTULO \\
\cline { 2 - 2 } & 1 \\
\hline
\end{tabular}

\section{Considerações iniciais}

Neste capítulo vamos introduzir algumas notações e resultados que irão nortear o desenvolvimento do trabalho.

Começamos apresentando o sistema de equações cuja resolubilidade global estamos interessados em estudar. Em seguida, com base no trabalho [BKNZ], definimos o espaço de recobrimento minimal do toro $\mathbb{T}^{n}$ com relação a uma 1-forma real, fechada e suave. Por fim, faremos uma breve exposição sobre um conceito muito importante para este trabalho, a saber, os números de Liouville.

\subsection{O sistema de equações}

Seja $c(t)=c_{1}(t) d t_{1}+\cdots+c_{n}(t) d t_{n}$ uma 1-forma complexa fechada de classe $C^{\infty}$ definida no toro $n$-dimensional $\mathbb{T}^{n}$. Considere o subfibrado vetorial $T^{\prime}$ gerado pela 1-forma $d x-c \in \bigwedge^{1} C^{\infty}\left(\mathbb{T}^{n} \times \mathbb{S}^{1}\right) \simeq \bigwedge^{1} C^{\infty}\left(\mathbb{T}^{n+1}\right)$ na qual $x$ denota a variável em $\mathbb{S}^{1}$. Portanto $T^{\prime}$ é um subfibrado de posto 1 do fibrado cotangente complexificado $\mathbb{C} \otimes T^{*}\left(\mathbb{T}^{n+1}\right)$ e o ortogonal $\mathcal{V}=\left(T^{\prime}\right)^{\perp}$ é um subfibrado vetorial de posto $n$ do fibrado tangente complexificado $\mathbb{C} \otimes T\left(\mathbb{T}^{n+1}\right)$. Além disso, $\mathcal{V}$ é gerado pelos campos vetoriais

$$
L_{j}=\frac{\partial}{\partial t_{j}}+c_{j}(t) \frac{\partial}{\partial x}, \quad j=1, \ldots, n
$$


sendo $(t, x)=\left(t_{1}, \ldots, t_{n}, x\right)$ as coordenadas em $\mathbb{T}^{n+1}$. Definido desta forma, o subfibrado $\mathcal{V}$ é uma estrutura localmente integrável de codimensão 1 sobre $\mathbb{T}^{n+1}$.

Como $c$ é uma 1-forma fechada temos

$$
\frac{\partial}{\partial t_{j}} c_{k}=\frac{\partial}{\partial t_{k}} c_{j}, \quad j, k=1, \ldots, n
$$

Portanto, os campos vetoriais (1.1) satisfazem

$$
\left[L_{j}, L_{k}\right] v \doteq L_{j}\left(L_{k} v\right)-L_{k}\left(L_{j} v\right)=\left(\frac{\partial}{\partial t_{j}} c_{k}-\frac{\partial}{\partial t_{k}} c_{j}\right) \frac{\partial}{\partial x} v=0, \quad v \in C^{\infty}\left(\mathbb{T}^{n+1}\right) .
$$

Uma exposição mais completa dessas ideias e conceitos encontra-se nos livros [T2] de Treves e $[\mathrm{BCH}]$ de Berhanu, Cordaro e Hounie.

Além disso, o sistema (1.1) dá origem a um complexo diferencial de operadores $\mathbb{L}$ o qual age, no primeiro nível, do seguinte modo

$$
\mathbb{L} u=d_{t} u+c(t) \wedge \frac{\partial}{\partial x} u, \quad u \in C^{\infty}\left(\mathbb{T}^{n+1}\right)\left(\text { ou } u \in \mathcal{D}^{\prime}\left(\mathbb{T}^{n+1}\right)\right),
$$

sendo $d_{t}$ a derivada exterior em $\mathbb{T}_{t}^{n}$.

Nosso interesse é a resolubilidade global no primeiro nível desse complexo; mais precisamente, estudamos a resolubilidade global da equação $\mathbb{L} u=f \operatorname{com} f \in \bigwedge^{1} C^{\infty}\left(\mathbb{T}^{n+1}\right)$ e $u \in \mathcal{D}^{\prime}\left(\mathbb{T}^{n+1}\right)$. Para que essa equação tenha solução é necessário que $f$ seja da seguinte forma

$$
f=\sum_{j=1}^{n} f_{j}(t, x) d t_{j}
$$

e além disso,

$$
L_{j} u=f_{j}, \quad j=1, \ldots, n
$$

\subsection{Condições de compatibilidade}

O intuito desta seção é estabelecer critérios para a escolha das 1-formas $f$ em $\bigwedge^{1} C^{\infty}\left(\mathbb{T}^{n+1}\right)$ de modo que faça sentido a questão da existência de solução da equação $\mathbb{L} u=f$.

Consideramos inicialmente as séries parciais de Fourier de $u$ e $f$ dadas por

$$
u(t, x)=\sum_{\xi \in \mathbb{Z}} \hat{u}(t, \xi) e^{i \xi x} \quad \text { e } \quad f(t, x)=\sum_{\xi \in \mathbb{Z}} \hat{f}(t, \xi) e^{i \xi x}
$$

sendo $\hat{f}(t, \xi)=\sum_{j=1}^{n} \hat{f}_{j}(t, \xi) d t_{j}$. No caso em que $u$ é função (integrável), a expressão $\hat{u}(t, \xi)$ é dada por

$$
\hat{u}(t, \xi)=\frac{1}{2 \pi} \int_{0}^{2 \pi} u(t, s) e^{-i \xi s} d s
$$


Se $u \in \mathcal{D}^{\prime}\left(\mathbb{T}^{n+1}\right)$ então, para cada $\xi$, o coeficiente $\hat{u}(\cdot, \xi)$ é uma distribuição dada por

$$
\langle\hat{u}(\cdot, \xi), \phi\rangle \doteq \frac{1}{2 \pi}\left\langle u, \phi \otimes e_{-\xi}\right\rangle, \quad \phi \in C^{\infty}\left(\mathbb{T}_{t}^{n}\right)
$$

sendo $\phi \otimes e_{-\xi}(t, x)=\phi(t) e^{-i \xi x} \in C^{\infty}\left(\mathbb{T}^{n+1}\right)$.

Quando os coeficientes $\hat{u}(\cdot, \xi)$ são funções suaves em $\mathbb{T}^{n}$, temos a seguinte caracterização: a expressão $u$ dada por (1.2) é uma função suave se, e somente se, para todo $\alpha \in \mathbb{Z}_{+}^{n}$ e todo $N \in \mathbb{N}$ vale

$$
\sup _{\xi \in \mathbb{Z}}\left\{\max _{t \in \mathbb{T}^{n}}\left\{\left|\partial^{\alpha} \hat{u}(t, \xi)\right|(1+|\xi|)^{N}\right\}\right\}<\infty
$$

Se $u \in \mathcal{D}^{\prime}\left(\mathbb{T}^{n+1}\right)$ então existem $C>0$ e $N \in \mathbb{N}$ de forma que

$$
|\langle\hat{u}(\cdot, \xi), \phi\rangle| \leq C\left|p_{N}(\phi)\right|(1+|\xi|)^{N}, \quad \forall \phi \in C^{\infty}\left(\mathbb{T}^{n}\right), \forall \xi \in \mathbb{Z}
$$

sendo

$$
p_{N}(\phi)=\sup \left\{\left|\partial^{\alpha} \phi(t)\right| ; t \in \mathbb{T}^{n} \text { e }|\alpha| \leq N\right\}
$$

Como a 1-forma $c$ é fechada, pelo Teorema de Fubini temos

$$
\begin{array}{r}
\int_{0}^{2 \pi}\left[c_{1}\left(s_{1}, t_{2}, \ldots, t_{n}\right)-c_{1}\left(s_{1}, t_{2}, \ldots, t_{n-1}, 0\right)\right] d s_{1} \\
=\int_{0}^{2 \pi} \int_{0}^{t_{n}} \frac{\partial}{\partial s_{n}} c_{1}\left(s_{1}, t_{2}, \ldots, t_{n-1}, s_{n}\right) d s_{n} d s_{1} \\
=\int_{0}^{t_{n}} \int_{0}^{2 \pi} \frac{\partial}{\partial s_{n}} c_{1}\left(s_{1}, t_{2}, \ldots, t_{n-1}, s_{n}\right) d s_{1} d s_{n} \\
=\int_{0}^{t_{n}} \int_{0}^{2 \pi} \frac{\partial}{\partial s_{1}} c_{n}\left(s_{1}, t_{2}, \ldots, t_{n-1}, s_{n}\right) d s_{1} d s_{n}=0
\end{array}
$$

repetindo este procedimento para as demais variáveis é possível concluir que

$$
\int_{0}^{2 \pi}\left[c_{1}\left(s_{1}, t_{2}, \ldots, t_{n}\right)-c_{1}\left(s_{1}, 0, \ldots, 0\right)\right] d s_{1}=0,
$$

para todo $\left(t_{2}, \ldots, t_{n}\right)$. Isto nos leva a definir as médias

$$
c_{j 0}=\frac{1}{2 \pi} \int_{0}^{2 \pi} c_{j}\left(0, \ldots, s_{j}, \ldots, 0\right) d s_{j}, \quad j=1, \ldots, n .
$$

Dando continuidade, escrevemos a 1-forma complexa $c$ no seguinte formato

$$
c=c_{0}+d_{t} C
$$


no qual $C$ é uma função suave de $t \in \mathbb{T}_{t}^{n}$ a valores complexos e $c_{0} \in \Lambda^{1} \mathbb{C}^{n}$. Identificamos a 1 -forma $c_{0}$ com o vetor $\left(c_{10}, \ldots, c_{n 0}\right)$ em $\mathbb{C}^{n}$ consistindo das médias $c_{j 0}=a_{j 0}+i b_{j 0}$ sendo

$$
a_{j 0}=\frac{1}{2 \pi} \int_{0}^{2 \pi} a_{j}\left(0, \ldots, s_{j}, \ldots, 0\right) d s_{j} \text { e } b_{j 0}=\frac{1}{2 \pi} \int_{0}^{2 \pi} b_{j}\left(0, \ldots, s_{j}, \ldots, 0\right) d s_{j} .
$$

Usaremos também as notações $a_{0}=\left(a_{10}, \ldots, a_{n 0}\right)$ e $b_{0}=\left(b_{10}, \ldots, b_{n 0}\right)$.

Lema 1.1. Se existe uma solução $u \in \mathcal{D}^{\prime}\left(\mathbb{T}^{n+1}\right) d a$ equação $\mathbb{L} u=f$ com $f \in$ $\bigwedge^{1} C^{\infty}\left(\mathbb{T}^{n+1}\right)$ ent $\tilde{a} o$

$$
\mathbb{L} f=0
$$

$e$

$$
\hat{f}(t, \xi) e^{i \xi\left(c_{0} \cdot t+C(t)\right)} \text { é exata sempre que } \xi \in \mathbb{Z} \text { satisfaz } \xi c_{0} \in \mathbb{Z}^{n} .
$$

Demonstração. A validade de (1.3) é consequência de que $\mathbb{L}$ gera um complexo e que $\mathbb{L} f=\mathbb{L}(\mathbb{L} u)$. Para maior clareza apresentamos a seguir os cálculos correspondentes. Uma vez que $\mathbb{L} u=L_{1} u d t_{1}+\cdots+L_{n} u d t_{n}$, obtemos

$$
\mathbb{L}(\mathbb{L} u)=\sum_{k=1}^{n-1} \sum_{j>k}^{n}\left(L_{k}\left(L_{j} u\right)-L_{j}\left(L_{k} u\right)\right) d t_{k} \wedge d t_{j}=\sum_{k=1}^{n-1} \sum_{j>k}^{n}\left[L_{k}, L_{j}\right](u) d t_{k} \wedge d t_{j}=0,
$$

ou seja, $0=\mathbb{L}(\mathbb{L} u)=\mathbb{L} f$.

Considere agora as séries de Fourier em relação à variável $x$ dadas por (1.2). Então, da equação $\mathbb{L} u=f$ temos

$$
d_{t} \hat{u}(t, \xi)+i \xi c(t) \wedge \hat{u}(t, \xi)=\hat{f}(t, \xi), \quad \xi \in \mathbb{Z},
$$

ou equivalentemente

$$
\left(d_{t}+i \xi c_{0} \wedge\right) e^{i \xi C(t)} \hat{u}(t, \xi)=e^{i \xi C(t)} \hat{f}(t, \xi), \quad \xi \in \mathbb{Z} .
$$

Seja $\xi \in \mathbb{Z}$ tal que $\xi c_{0} \in \mathbb{Z}^{n}$; assim $e^{i \xi c_{0} \cdot t}$ é bem definido para $t \in \mathbb{T}^{n} \mathrm{e}$

$$
d_{t}\left(e^{i \xi\left(c_{0} \cdot t+C(t)\right)} \hat{u}(t, \xi)\right)=e^{i \xi\left(c_{0} \cdot t+C(t)\right)} \hat{f}(t, \xi),
$$

portanto, $e^{i \xi\left(c_{0} \cdot t+C(t)\right)} \hat{f}(t, \xi)$ é exata.

Motivados pelo lema anterior, definimos o seguinte espaço

$$
\mathbb{E}=\left\{f \in \bigwedge^{1} C^{\infty}\left(\mathbb{T}^{n+1}\right) ; \mathbb{L} f=0 \text { e vale }(1.4)\right\} .
$$

Definição 1.2. Dizemos que o operador diferencial $\mathbb{L}$ é globalmente resolúvel em $\mathbb{T}^{n+1}$ se para cada $f \in \mathbb{E}$ existe $u \in \mathcal{D}^{\prime}\left(\mathbb{T}^{n+1}\right)$ tal que $\mathbb{L} u=f$. 


\subsection{Recobrimento minimal de $\mathbb{T}^{n}$}

Dada uma 1-forma real, fechada e suave $b$ definida no toro $\mathbb{T}^{n} \simeq(\mathbb{R} / 2 \pi \mathbb{Z})^{n}$, vamos apresentar um conceito que chamaremos de recobrimento minimal de $\mathbb{T}^{n}$ com relação a $b$. Esse conceito foi introduzido primeiramente no trabalho [BKNZ]. A 1-forma $b \in \bigwedge^{1} C^{\infty}\left(\mathbb{T}^{n} ; \mathbb{R}\right)$ será neste trabalho a parte imaginária da 1-forma complexa $c$ considerada na seção anterior; ou seja, escrevemos $c=a+i b$ sendo $a(t)=\sum_{j=1}^{n} a_{j}(t) d t_{j} \mathrm{e}$ $b(t)=\sum_{j=1}^{n} b_{j}(t) d t_{j} 1$-formas reais sobre $\mathbb{T}^{n}$.

O recobrimento minimal dependerá dos períodos

$$
b_{j 0}=\frac{1}{2 \pi} \int_{0}^{2 \pi} b_{j}\left(0, \ldots, s_{j}, \ldots, 0\right) d s_{j}, \quad j=1, \ldots, n,
$$

das funções $b_{j}$.

Considere o grupo de períodos da 1-forma $b$ definido por

$$
\operatorname{Per}(b) \doteq\left\{\frac{1}{2 \pi} \int_{\sigma} b ; \quad \sigma \text { é um 1-ciclo em } \mathbb{T}^{n}\right\}
$$

Equivalentemente, $\operatorname{Per}(b)$ é o subgrupo aditivo de $\mathbb{R}$ consistindo de todas as combinações inteiras dos números $b_{10}, \ldots, b_{n 0}$. Assim, se $r \doteq \operatorname{posto}(\operatorname{Per}(b))$ temos que $r$ é o maior número de períodos racionalmente independentes dentre $b_{10}, \ldots, b_{n 0}$. Logo, as possibilidades para posto $(\operatorname{Per}(b))$ são $r=0,1, \ldots, n$.

Dados dois recobrimentos $\Pi_{1}: \mathcal{T}_{1} \rightarrow \mathbb{T}^{n}$ e $\Pi_{2}: \mathcal{T}_{2} \rightarrow \mathbb{T}^{n}$ do toro $\mathbb{T}^{n}$ tais que os respectivos pull-backs $\Pi_{1}^{*} b$ e $\Pi_{2}^{*} b$ são formas exatas, escrevemos $\mathcal{T}_{1} \preceq \mathcal{T}_{2}$ quando $\mathcal{T}_{2}$ for um recobrimento de $\mathcal{T}_{1}$. Definido dessa forma, $\preceq$ é uma relação de ordem parcial.

Definição 1.3. Seja b uma 1-forma real, suave e fechada definida em $\mathbb{T}^{n}$. Um recobrimento minimal do toro $\mathbb{T}^{n}$ com relação a b é um espaço de recobrimento de $\mathbb{T}^{n}$ que é minimal no sentido da relação $\preceq$.

Se $\mathcal{T}$ é um recobrimento minimal de $\mathbb{T}^{n}$ com relação à uma 1 -forma real, fechada e suave $b$ então $\mathcal{T}$ é isomorfo a $\mathbb{T}^{n-r} \times \mathbb{R}^{r}$, sendo $r$ o posto do grupo de períodos da 1-forma $b$.

Daqui em diante, em vez de dizer um recobrimento minimal vamos dizer o recobrimento minimal de $\mathbb{T}^{n}$ e escrever $\mathcal{T}=\mathbb{T}^{n-r} \times \mathbb{R}^{r}$.

Veja que $r=0$ se, e somente se, a 1 -forma $b$ é exata e neste caso $\mathcal{T}=\mathbb{T}^{n}$. O outro caso extremo será quando $r=n$. Neste caso $\mathcal{T}=\mathbb{R}^{n}$ e $\Pi: \mathbb{R}^{n} \rightarrow \mathbb{T}^{n}$ é o recobrimento universal de $\mathbb{T}^{n}$.

Essa definição nos interessa pois garante a existência de uma primitiva global da 1 -forma $\Pi^{*} b$ definida no recobrimento minimal $\mathcal{T}$. 
Se a 1-forma $b$ é exata, além de possuir uma primitiva global em $\mathbb{T}^{n}$, o pull-back de $b$ em $\mathbb{R}^{m} \times \mathbb{T}^{n-m}, m=1, \ldots, n$, ainda é uma 1 -forma exata e, portanto, possui primitiva global nesses espaços. Poderíamos pensar em estudar a resolubilidade global usando um deles, porém no caso em que $b$ é exata pode ser essencial o uso da definição do recobrimento minimal conforme mostra o exemplo a seguir.

Se $b\left(t_{1}, t_{2}\right)=i \cos t_{2} d t_{2}$, então o sistema

$$
\left\{\begin{array}{l}
L_{1}=\frac{\partial}{\partial t_{1}} \\
L_{2}=\frac{\partial}{\partial t_{2}}+i \cos t_{2} \frac{\partial}{\partial x}
\end{array}\right.
$$

é globalmente resolúvel em $\mathbb{T}^{3}$ (pelo trabalho $[\mathrm{CH}]$ ) pois $B\left(t_{1}, t_{2}\right)=$ sen $t_{2}$ é uma primitiva global de $b$ que tem todos os subníveis e superníveis conexos em $\mathbb{T}^{2}$, porém em $\mathbb{T}_{t_{1}}^{1} \times \mathbb{R}_{t_{2}}$ e em $\mathbb{R}^{2}$ existem subníveis e superníveis desconexos das respectivas primitivas. Ou seja, a propriedade de conexidade de todos os subníveis e superníveis depende de onde estamos considerando a primitiva global da 1-forma $b$.

Se $b=b_{1} d t_{1}+b_{2} d t_{2}$ é uma 1-forma real fechada e suave definida em $\mathbb{T}^{2}$, os trabalhos $[\mathrm{CH}\rfloor$ (quando $r=0$ ), $[\mathrm{BKNZ}\rfloor$ (quando $r=1$ ) e $[\mathrm{BK}\rfloor$ (quando $r=2$ ) nos mostram que a resolubilidade global do operador

$$
\mathbb{L}=d_{t}+i b(t) \wedge \frac{\partial}{\partial x}
$$

é equivalente à propriedade de todos os conjuntos de subnível e supernível de uma primitiva global de $\Pi^{*} b$ serem conexos no recobrimento minimal $\mathcal{T}$.

Aproveitamos para provar nesta seção um resultado de normalização para os perío$\operatorname{dos} b_{10}, \ldots, b_{n 0}$. Vamos denotar por $s \doteq n-r$ o coposto do grupo de períodos $\operatorname{Per}(b)$, sendo $r=\operatorname{posto}(\operatorname{Per}(b))$.

Se $b$ for uma 1-forma exata então $r=0$ ou equivalentemente $\left(b_{10}, \ldots, b_{n 0}\right)=0$. Se a 1-forma $b$ não for exata temos

$$
1 \leq r \leq n \quad \text { e } \quad 0 \leq s \leq n-1
$$

e vale o seguinte resultado:

Lema 1.4. Seja $b=\sum_{j=1}^{n} b_{j} d t_{j} \in \bigwedge^{1} C^{\infty}\left(\mathbb{T}^{n} ; \mathbb{R}\right)$ uma 1-forma fechada. Se b não é exata então existe um sistema de coordenadas em $\mathbb{T}^{n}$ no qual os periodos da 1-forma $b$ satisfazem:

(i) $b_{10}=\cdots=b_{s 0}=0$ e $b_{n 0}<\cdots<b_{(s+1) 0}<0$, quando $s \geq 1$; 
(ii) $b_{n 0}<\cdots<b_{10}<0$, quando $s=0$.

Demonstração. Seja $B$ uma primitiva global do pull-back $\Pi^{*} b$ através do recobrimento universal $\Pi: \mathbb{R}^{n} \rightarrow \mathbb{T}^{n}$. Podemos escrever $B(t)=b_{0} \cdot t+P(t)$ sendo $P$ uma função suave e $2 \pi$-periódica.

Começamos com o caso $s \geq 1$. Neste caso, os períodos $b_{10}, \ldots, b_{n 0}$ são racionalmente dependentes.

Afirmação: existe um sistema de coordenadas em $\mathbb{T}^{n}$ tal que $b_{10}=0$. De fato, se existir algum período $b_{j 0}=0$ basta considerar o difeomorfismo $\left(t_{1}, \ldots, t_{j}, \ldots, t_{n}\right) \mapsto$ $\left(t_{j}, \ldots, t_{1}, \ldots, t_{n}\right)$. Caso isso não ocorra, podemos escrever algum período $b_{j 0}$ como combinação racional dos demais. Logo, existem $k_{1}, \ldots, k_{n} \in \mathbb{Z}$ tais que $\operatorname{mdc}\left(k_{1}, \ldots, k_{n}\right)=$ $1 \mathrm{e}$

$$
k_{1} b_{10}+\cdots+k_{n} b_{n 0}=0
$$

Seja $d=\operatorname{mdc}\left(k_{1}, k_{n}\right)$, então existem inteiros $p$ e $q$ satisfazendo $d=p k_{1}+q k_{n}, \operatorname{logo}$ $1=p \alpha+q \beta$ sendo $\alpha=k_{1} / d$ e $\beta=k_{n} / d$. Considere o difeomorfismo $t \mapsto t^{\prime} \doteq A_{1}^{-1} t$ no qual

$$
A_{1}=\left(\begin{array}{ccccc}
\alpha & 0 & \cdots & 0 & -q \\
0 & 1 & 0 & \cdots & 0 \\
\vdots & 0 & \ddots & & \vdots \\
0 & \vdots & & 1 & 0 \\
\beta & 0 & \cdots & 0 & p
\end{array}\right) .
$$

Note que este difeomorfismo fica bem definido uma vez que $A_{1} \in S L(n ; \mathbb{Z})$, sendo $S L(n ; \mathbb{Z})$ o grupo das matrizes de ordem $n$, com entradas inteiras, invertíveis e com determinante igual a 1 .

Além disso,

$$
\begin{array}{r}
b_{0} \cdot t\left(t^{\prime}\right)=\left(\alpha t_{1}^{\prime}-q t_{n}^{\prime}\right) b_{10}+t_{2}^{\prime} b_{20}+\cdots+t_{n-1}^{\prime} b_{(n-1) 0}+\left(\beta t_{1}^{\prime}+p t_{n}^{\prime}\right) b_{n 0} \\
=t_{1}^{\prime}\left(\alpha b_{10}+\beta b_{n 0}\right)+t_{2}^{\prime} b_{20}+\cdots+t_{n-1}^{\prime} b_{(n-1) 0}+t_{n}^{\prime}\left(p b_{n 0}-q b_{10}\right),
\end{array}
$$

ou seja, nas novas coordenadas temos

$$
b_{10}^{\prime}=\alpha b_{10}+\beta b_{n 0}, \quad b_{j 0}^{\prime}=b_{j 0}, j=2, \ldots, n-1, \quad b_{n 0}^{\prime}=p b_{n 0}-q b_{10} .
$$

Note agora que, tomando $k_{1}^{\prime}=d, k_{j}^{\prime}=k_{j}, j=2, \ldots, n-1$, e $k_{n}^{\prime}=0$ temos $\operatorname{mdc}\left(k_{1}^{\prime}, \ldots, k_{n-1}^{\prime}\right)=1$ e além disso por (1.6) obtemos

$$
\begin{array}{r}
k_{1}^{\prime} b_{10}^{\prime}+\cdots+k_{n-1}^{\prime} b_{(n-1) 0}^{\prime}= \\
d\left(\alpha b_{10}+\beta b_{n 0}\right)+k_{2} b_{20}+\cdots+k_{n-1} b_{(n-1) 0}=0 .
\end{array}
$$


Isto significa que nas novas coordenadas os períodos $b_{10}^{\prime}, \ldots, b_{(n-1) 0}^{\prime}$ são racionalmente dependentes.

Para não sobrecarregar a notação voltamos a escrever $t=t^{\prime}$ e $k_{j}=k_{j}^{\prime}$ para $j=$ $1, \ldots, n-1$. Assim, existem inteiros $k_{1}, \ldots, k_{n-1}$ tais que $\operatorname{mdc}\left(k_{1}, \ldots, k_{n-1}\right)=1 \mathrm{e}$ $k_{1} b_{10}+\cdots+k_{n-1} b_{(n-1) 0}=0$.

Seja agora $d=\operatorname{mdc}\left(k_{1}, k_{n-1}\right)$, então existem inteiros $p$ e $q$ tais que $d=p k_{1}+q k_{n-1}$, daqui $1=p \alpha+q \beta$ sendo $\alpha=k_{1} / d$ e $\beta=k_{n-1} / d$. Considere o difeomorfismo $t \mapsto t^{\prime} \doteq$ $A_{2}^{-1} t$ no qual

$$
A_{2}=\left(\begin{array}{ccccc}
\alpha & 0 & \cdots & -q & 0 \\
0 & 1 & 0 & \cdots & 0 \\
\vdots & 0 & \ddots & & \vdots \\
\beta & \vdots & & p & 0 \\
0 & 0 & \cdots & 0 & 1
\end{array}\right)
$$

Novamente $A_{2} \in S L(n ; \mathbb{Z})$ e obtemos

$$
\begin{array}{r}
b_{0} \cdot t\left(t^{\prime}\right)=\left(\alpha t_{1}^{\prime}-q t_{n-1}^{\prime}\right) b_{10}+t_{2}^{\prime} b_{20}+\cdots+\left(\beta t_{1}^{\prime}+p t_{n-1}^{\prime}\right) b_{(n-1) 0}+t_{n}^{\prime} b_{n 0} \\
=t_{1}^{\prime}\left(\alpha b_{10}+\beta b_{(n-1) 0}\right)+t_{2}^{\prime} b_{20}+\cdots+t_{n-1}^{\prime}\left(p b_{(n-1) 0}-q b_{10}\right)+t_{n}^{\prime} b_{n 0}
\end{array}
$$

ou seja, nas novas coordenadas $t_{1}^{\prime}, \ldots, t_{n}^{\prime}$ temos

$$
\begin{gathered}
b_{10}^{\prime}=\alpha b_{10}+\beta b_{(n-1) 0}, \quad b_{j 0}^{\prime}=b_{j 0}, j=2, \ldots, n-2, n, \\
b_{(n-1) 0}^{\prime}=p b_{(n-1) 0}-q b_{10} .
\end{gathered}
$$

Escolhemos $k_{1}^{\prime}=d, k_{j}^{\prime}=k_{j}, j=2, \ldots, n-2$ e $k_{n-1}^{\prime}=k_{n}^{\prime}=0$ e concluímos que $\operatorname{mdc}\left(k_{1}^{\prime}, \ldots, k_{n-2}^{\prime}\right)=1 \mathrm{e}$

$$
k_{1}^{\prime} b_{10}^{\prime}+\cdots+k_{n-2}^{\prime} b_{(n-2) 0}^{\prime}=0 .
$$

Assim por diante, quando $n>2$, construímos matrizes $A_{1}, \ldots, A_{n-2} \in S L(n ; \mathbb{Z})$ e um difeomorfismo $t \mapsto A_{n-2}^{-1} \ldots A_{1}^{-1} t$ de forma que no novo sistema de coordenadas (também denotado por $t_{1}, \ldots, t_{n}$ ) o período $b_{10}$ é um múltiplo racional de $b_{20}$. Isto é, existem inteiros $k_{1}$ e $k_{2}$ tais que $\operatorname{mdc}\left(k_{1}, k_{2}\right)=1 \mathrm{e}$

$$
k_{1} b_{10}+k_{2} b_{20}=0 \text {. }
$$


Para finalizar nossa afirmação, considere inteiros $p$ e $q$ tais que $1=p k_{1}+q k_{2}$. Definimos o difeomorfismo $t \mapsto t^{\prime} \doteq A_{n-1}^{-1} t$ no qual

$$
A_{n-1}=\left(\begin{array}{ccccc}
k_{1} & -q & \cdots & 0 & 0 \\
k_{2} & p & 0 & \cdots & 0 \\
\vdots & 0 & \ddots & & \vdots \\
0 & \vdots & & 1 & 0 \\
0 & 0 & \cdots & 0 & 1
\end{array}\right)
$$

Note que este difeomorfismo fica bem definido pois $A_{n-1} \in S L(n ; \mathbb{Z})$. Além disso,

$$
\begin{aligned}
& b_{0} \cdot t\left(t^{\prime}\right)=\left(k_{1} t_{1}^{\prime}-q t_{2}^{\prime}\right) b_{10}+\left(k_{2} t_{1}^{\prime}+p t_{2}^{\prime}\right) b_{20}+\cdots+t_{n}^{\prime} b_{n 0} \\
= & t_{1}^{\prime}\left(k_{1} b_{10}+k_{2} b_{20}\right)+t_{2}^{\prime}\left(p b_{20}-q b_{10}\right)+t_{3}^{\prime} b_{30}+\cdots+t_{n}^{\prime} b_{n 0},
\end{aligned}
$$

ou seja, nas novas coordenadas temos

$$
b_{10}^{\prime}=k_{1} b_{10}+k_{2} b_{20}=0, \quad b_{20}^{\prime}=p b_{20}-q b_{10}, \quad b_{j 0}^{\prime}=b_{j 0}, j=3, \ldots, n .
$$

Dessa forma, concluímos a prova da afirmação feita no início desta demonstração. Este processo pode ser repetido $s=n-r$ vezes de modo a obter $b_{10}=\cdots=b_{s 0}=0 \mathrm{e}$ $b_{(s+1) 0}, \ldots, b_{n 0}$ racionalmente independentes.

Por fim, considere a matriz

$$
A=\left(a_{i j}\right)=\left\{\begin{array}{ll}
0 & \text { se } i \neq j \\
1 & \text { se } i=j=1, \ldots, s \\
1 & \text { se } i=j \text { e } b_{j 0}<0 \\
-1 & \text { se } i=j \text { e } b_{j 0}>0
\end{array} .\right.
$$

Então, a aplicação $t \mapsto t^{\prime}=A^{-1} t$ define um difeomorfismo que produz coordenadas em $\mathbb{T}^{n}$ onde $b_{10}=\cdots=b_{s 0}=0$ e $b_{j 0}<0, j=s+1, \ldots, n$.

A ordenação $b_{n 0}<\cdots<b_{(s+1) 0}<0$, para $s \geq 0$, mencionada em $(i)$ e (ii) pode ser obtida reordenando as coordenadas em $\mathbb{T}^{n}$.

\subsection{Números de Liouville}

Utilizamos neste trabalho um importante conceito da teoria dos números, os chamados números de Liouville (ver [MMST] página 356). No entanto, como vamos trabalhar 
com sistemas de mais de um campo, precisamos definir uma generalização desse conceito para vetores do $\mathbb{R}^{N}$.

Dado $\alpha \in \mathbb{R} \backslash \mathbb{Q}$, dizemos que $\alpha$ é um número de Liouville se para cada $m \in \mathbb{N}$ existem infinitos racionais $p / q$ satisfazendo

$$
\left|\alpha-\frac{p}{q}\right|<\frac{1}{q^{m}}
$$

ou equivalentemente, $\alpha \in \mathbb{R} \backslash \mathbb{Q}$ é um número de Liouville se existir uma sequência $p_{l} / q_{l}$ de números racionais tal que

$$
\left|\alpha-\frac{p_{l}}{q_{l}}\right|<\frac{1}{\left(q_{l}\right)^{l}}, \quad \forall l \in \mathbb{N} .
$$

Definição 1.5. Dado $\alpha=\left(\alpha_{1}, \ldots, \alpha_{N}\right) \in \mathbb{R}^{N} \backslash \mathbb{Q}^{N}$, dizemos que $\alpha$ é Liouville se existem uma constante $C>0$ e uma sequência $\left\{\left(\kappa_{l}, \xi_{l}\right)\right\}$ em $\mathbb{Z}^{N} \times \mathbb{Z}\left(\xi_{l} \geq 2\right)$ tais que

$$
\max _{j=1, \ldots, N}\left|\alpha_{j}-\frac{\kappa_{l}^{(j)}}{\xi_{l}}\right|<\frac{C}{\left(\xi_{l}\right)^{l}}, \quad \forall l \in \mathbb{N} .
$$

Essa definição é equivalente a uma definição análoga com a constante $C=1$.

Se $\alpha \notin \mathbb{Q}^{N}$ é Liouville então pela Definição 1.5 cada $\alpha_{j} \notin \mathbb{Q}$ é um número de Liouville, porém se cada $\alpha_{j}$ é um número de Liouville, não necessariamente o vetor $\alpha$ é Liouville. No trabalho [B] de Bergamasco é apresentado um exemplo (Exemplo 4.9) na classe exponencial Liouville onde $\alpha_{1}$ e $\alpha_{2}$ são exponenciais Liouville e, portanto, números de Liouville, no entanto o vetor $\left(\alpha_{1}, \alpha_{2}\right)$ não é exponencial Liouville. Mais ainda, o autor mostra que o vetor $\left(\alpha_{1}, \alpha_{2}\right)$ não é sequer Liouville no sentido da Definição 1.5 .

Lema 1.6. Se $\alpha=\left(\alpha_{1}, \ldots, \alpha_{N}\right) \notin \mathbb{Q}^{N}$ é Liouville então existem uma constante $C>0$ e uma sequência $\left\{\left(\kappa_{l}, \xi_{l}\right)\right\}$ em $\mathbb{Z}^{N} \times \mathbb{Z}\left(\xi_{l} \geq 2\right)$ tais que

$$
\max _{j=1, \ldots, N}\left|\alpha_{j}-\frac{\kappa_{l}^{(j)}}{\xi_{l}}\right|<\frac{C}{\left|\left(\kappa_{l}, \xi_{l}\right)\right|^{l}}, \quad \forall l \in \mathbb{N} .
$$

Demonstração. Sejam $C>0$ a constante e $\left\{\left(\kappa_{l}, \xi_{l}\right)\right\}$ a sequência obtidas pela Definição 1.5. Note que

$$
\left|k_{l}^{(j)}\right|<C\left(\xi_{l}\right)^{1-l}+\left|\alpha_{j}\right| \xi_{l} \leq \xi_{l}(C+|\alpha|), \quad j=1, \ldots, N, \quad \forall l \in \mathbb{N} .
$$

Assim, $\left|\left(\kappa_{l}, \xi_{l}\right)\right| \leq\left|\kappa_{l}^{(1)}\right|+\cdots+\left|\kappa_{l}^{(N)}\right|+\left|\xi_{l}\right|<\widetilde{C} \xi_{l}$ sendo $\widetilde{C}=1+N(C+|\alpha|)$ e segue facilmente a conclusão. 
Lema 1.7. Sejam $\alpha=\left(\alpha_{1}, \ldots, \alpha_{N}\right) \notin \mathbb{Q}^{N}$ e $\eta \in \mathbb{N}$. Se $\alpha$ é Liouville então existem uma constante $C>0$ e uma sequência $\left\{\left(\kappa_{l}, \xi_{l}\right)\right\}$ em $(\eta \mathbb{Z})^{N} \times \eta \mathbb{Z}\left(\xi_{l} \geq 2\right)$ tais que

$$
\max _{j=1, \ldots, N}\left|\alpha_{j}-\frac{\kappa_{l}^{(j)}}{\xi_{l}}\right|<\frac{C}{\left(\xi_{l}\right)^{l}}, \forall l \in \mathbb{N} .
$$

Demonstração. Como $\alpha$ é Liouville, por definição, existem uma constante $C>0$ e uma sequência $\left\{\left(\kappa_{l}, \xi_{l}\right)\right\}$ em $\mathbb{Z}^{N} \times \mathbb{Z}\left(\xi_{l} \geq 2\right)$ tais que

$$
\max _{j=1, \ldots, N}\left|\alpha_{j}-\frac{\kappa_{l}^{(j)}}{\xi_{l}}\right|<\frac{C}{\left(\xi_{l}\right)^{l}}, \forall l \in \mathbb{N}
$$

sendo que podemos assumir que $\xi_{l}<\xi_{l+1}, \forall l \in \mathbb{N}$.

Para $\eta=1$ a conclusão é imediata da Definição 1.5. Para $\eta>1$ temos $\eta \leq \xi_{\eta} \leq$ $\xi_{\eta l} \leq\left(\xi_{\eta l}\right)^{\eta-1}, \forall l \in \mathbb{N}$. Note que a constante $C$ anterior e a sequência $\left(\widetilde{\kappa}_{l}, \widetilde{\xi}_{l}\right) \doteq$ $\left(\eta \kappa_{\eta l}, \eta \xi_{\eta l}\right), l \in \mathbb{N}$, satisfazem a condição desejada. Com efeito,

$$
\max _{j=1, \ldots, N}\left|\alpha_{j}-\frac{\eta \kappa_{\eta l}^{(j)}}{\eta \xi_{\eta l}}\right| \leq C\left(\xi_{\eta l}\right)^{-\eta l}=C \underbrace{\left(\eta^{l} \xi_{\eta l}^{-(\eta-1) l}\right)}_{\leq 1}\left(\eta \xi_{\eta l}\right)^{-l} \leq C\left(\eta \xi_{\eta l}\right)^{-l}, \quad \forall l \in \mathbb{N} .
$$





$\frac{20}{2}$

\section{Sistemas involutivos desacoplados}

Neste capítulo estudamos a resolubilidade global do operador $\mathbb{L}$ no caso em que cada função $b_{j}$ depende apenas da variável $t_{j}$ correspondente. Mais precisamente, consideramos o operador diferencial

$$
\mathbb{L}=d_{t}+(a(t)+i b(t)) \wedge \frac{\partial}{\partial x}
$$

com $a(t)=\sum_{j=1}^{n} a_{j}(t) d t_{j}$ e $b(t)=\sum_{j=1}^{n} b_{j}\left(t_{j}\right) d t_{j} 1$-formas reais. Esse operador está associado ao sistema de campos vetoriais

$$
L_{j}=\frac{\partial}{\partial t_{j}}+\left(a_{j}(t)+i b_{j}\left(t_{j}\right)\right) \frac{\partial}{\partial x}, \quad j=1, \ldots, n,
$$

no qual $a_{j} \in C^{\infty}\left(\mathbb{T}^{n} ; \mathbb{R}\right), b_{j} \in C^{\infty}\left(\mathbb{T}^{1} ; \mathbb{R}\right)$ e $(t, x)=\left(t_{1}, \ldots, t_{n}, x\right)$ são as coordenadas em $\mathbb{T}^{n+1} \simeq(\mathbb{R} / 2 \pi \mathbb{Z})^{n+1}$.

Considere o espaço de recobrimento minimal $\mathcal{T}=\mathbb{R}^{r} \times \mathbb{T}^{n-r}$ com relação à 1-forma real $b$, segundo a Definição 1.3. Assim, a 1 -forma $\Pi^{*} b$ possui uma primitiva global $B$ definida em $\mathcal{T}$. Uma vez que cada função $b_{j}$ depende apenas da variável $t_{j}$, a função $B: \mathcal{T} \rightarrow \mathbb{R}$ pode ser escrita na seguinte forma $B(t)=\sum_{j=1}^{n} B_{j}\left(t_{j}\right)$.

Sejam $J=\left\{j_{1}, \ldots, j_{m}\right\} \subset\{1, \ldots, n\} \operatorname{com} j_{1}<\cdots<j_{m}$ e $a_{0} \doteq\left(a_{10}, \ldots, a_{n 0}\right) \in \mathbb{R}^{n}$ sendo cada $a_{j 0}$ a média da função $a_{j}$. Quando $a_{0} \in \mathbb{Q}^{n}$ e $J \neq \emptyset$, denotamos por $q_{J}$ o menor inteiro positivo que satisfaz $q_{J}\left(a_{j_{1} 0}, \ldots, a_{j_{m} 0}\right) \in \mathbb{Z}^{m}$. No caso em que 
$J=\{1, \ldots, n\}$ usamos a notação $q_{*}$. Assim, qualquer que seja $J=\left\{j_{1}, \ldots, j_{m}\right\} \neq \emptyset$ com $1 \leq j_{1}<\cdots<j_{m} \leq n$ teremos $q_{J} \leq q_{*}$ e além disso $q_{J}$ divide $q_{*}$.

Daqui em diante vamos considerar o conjunto $J=\left\{j \in\{1, \ldots, n\} ; b_{j} \equiv 0\right\}$ e vamos escreve-lo da seguinte forma $J=\left\{j_{1}<\cdots<j_{m}\right\}$.

Sob estas notações, vamos enunciar o teorema que é o principal resultado deste capítulo.

Teorema 2.1. Seja B uma primitiva global de $\Pi^{*} b$ definida no recobrimento minimal $\mathcal{T}$. O operador $\mathbb{L}$ definido em (2.1) é globalmente resolúvel se, e somente se, pelo menos uma das duas situações ocorre:

I) $J \neq \emptyset e\left(a_{j_{1} 0}, \ldots, a_{j_{m} 0}\right) \notin \mathbb{Q}^{m}$ é não-Liouville.

II) Os subniveis $\Omega_{s}=\{t \in \mathcal{T} ; B(t)<s\}$ e superniveis $\Omega^{s}=\{t \in \mathcal{T} ; B(t)>s\}$ são conexos para todo $s \in \mathbb{R}$ e além disso uma das seguintes condições é satisfeita:

1. $J=\emptyset$, b é exata e $a_{0} \in \mathbb{Z}^{n}$;

2. $J \neq \emptyset, b$ é exata, $a_{0} \in \mathbb{Q}^{n}$ e $q_{J}=q_{*}$;

3. $b$ é não exata.

Note que quando $J=\{1, \ldots, n\}$ então $b=0$, portanto, $b$ é exata e $q_{J}=q_{*}$. Nestas condições, o teorema anterior diz que $\mathbb{L}$ é globalmente resolúvel se, e somente se, $a_{0} \notin \mathbb{Q}^{n}$ é não-Liouville ou $a_{0} \in \mathbb{Q}^{n}$, o que condiz com o resultado do trabalho de Bergamasco e Petronilho $\mid \mathrm{BP}]$.

Quando $b=\sum_{j=1}^{n} b_{j}\left(t_{j}\right) d t_{j}$ é uma 1-forma não exata, a propriedade de todos os subníveis e superníveis de uma primitiva global de $\Pi^{*} b$ serem conexos no recobrimento minimal $\mathcal{T}$ está intimamente ligada com a existência de uma função $b_{j} \not \equiv 0$ que não muda de sinal, conforme mostra o seguinte resultado:

Lema 2.2. Sejam $b=\sum_{j=1}^{n} b_{j}\left(t_{j}\right) d t_{j}$ uma 1-forma nãa exata e $B=\sum_{j=1}^{n} B_{j}\left(t_{j}\right)$ uma primitiva global de b definida no recobrimento minimal $\mathcal{T}=\mathbb{R}^{r} \times \mathbb{T}^{n-r}$. Então os subniveis $\Omega_{s}=\{t \in \mathcal{T} ; B(t)<s\}$ e superniveis $\Omega^{s}=\{t \in \mathcal{T} ; B(t)>s\}$ são conexos para todo $s \in \mathbb{R}$ se, e somente se, existe uma função $b_{j} \not \equiv 0$ que não muda de sinal.

Demonstração. Suponha que cada função $b_{j} \not \equiv 0$ muda de sinal. Então, cada primitiva $B_{j}: \mathbb{R} \rightarrow \mathbb{R}, j=1, \ldots, r$, tem um supernível desconexo em $\mathbb{R}$, logo existem um número real $s_{j}$ e um intervalo aberto limitado $I_{j}$ o qual é uma componente conexa de $\left\{t_{j} \in \mathbb{R} ; B_{j}\left(t_{j}\right)>s_{j}\right\}$. Escrevemos $I_{j}=\left(p_{j}, q_{j}\right)$ e $M_{j} \doteq \max \left\{B_{j}\left(t_{j}\right), t_{j} \in\left[p_{j}, q_{j}\right]\right\}$, $\operatorname{assim} s_{j}=B_{j}\left(p_{j}\right)=B_{j}\left(q_{j}\right)<M_{j}, j=1, \ldots, r$. Além disso, denotamos por $M^{\prime} \mathrm{o}$ máximo da função $B_{r+1}+\cdots+B_{n}$ sobre $\mathbb{T}^{n-r}$. 
Considere os conjuntos $I=I_{1} \times \cdots \times I_{r}$ e $U \doteq I \times \mathbb{T}^{n-r}$. Tomamos $t^{*}=\left(t_{1}^{*}, \ldots, t_{n}^{*}\right) \in$ $U$ tal que $B_{j}\left(t_{j}^{*}\right)=M_{j}, j=1, \ldots, r$ e $B_{r+1}\left(t_{r+1}^{*}\right)+\cdots+B_{n}\left(t_{n}^{*}\right)=M^{\prime}$, assim se $M \doteq B\left(t^{*}\right)$ então $B(t) \leq M$, para todo $t \in U$ e $B(t)<M$, para todo $t \in \partial I \times \mathbb{T}^{n-r}$.

Escolhemos agora $\widetilde{M}<s<M$ sendo $\widetilde{M} \doteq \max \left\{B(t) ; t \in \partial I \times \mathbb{T}^{n-r}\right\}$. Afirmamos que o supernível $\Omega^{s}$ é desconexo em $\mathcal{T}$. De fato, escrevendo $\Omega^{s}=\left(U \cap \Omega^{s}\right) \cup\left(\Omega^{s} \backslash U\right)$ temos $U \cap \Omega^{s} \neq \emptyset$ e $\Omega^{s} \backslash U \neq \emptyset$. Como $U$ é aberto em $\mathcal{T}$ então $U \cap \Omega^{s}$ é aberto em $\Omega^{s}$ (na topologia induzida por $\mathcal{T}$ ). Provaremos agora que $\Omega^{s} \backslash U$ também é aberto em $\Omega^{s}$.

Seja $t=\left(t^{\prime}, t^{\prime \prime}\right) \in \Omega^{s} \backslash U=\Omega^{s} \cap(\mathcal{T} \backslash U)$ sendo $t^{\prime}=\left(t_{1}, \ldots, t_{r}\right)$ e $t^{\prime \prime}=\left(t_{r+1}, \ldots, t_{n}\right)$, $\operatorname{logo} t^{\prime} \in \mathbb{R}^{r} \backslash I$. Portanto, existe uma vizinhança $V_{t^{\prime}}$ de $t^{\prime}$ tal que $V_{t^{\prime}} \subset \mathbb{R}^{r} \backslash I$. Caso contrário, teríamos $V_{t^{\prime}} \cap I \neq \emptyset$ para cada vizinhança $V_{t^{\prime}} \subset \mathbb{R}^{r}$ de $t^{\prime}$ o que implicaria em $t^{\prime} \in \partial I, \operatorname{assim} B(t) \leq \widetilde{M}<s$, o que é uma contradição. Definimos então $V_{t}=$ $V_{t^{\prime}} \times \mathbb{T}^{n-r} \subset \mathcal{T} \backslash U$. Além disso, pela continuidade da função $B$ existe uma vizinhança $W_{t} \subset \mathcal{T}$ de $t$ tal que $W_{t} \subset \Omega^{s}$. Portanto, $V_{t} \cap W_{t} \subset \Omega^{s} \cap(\mathcal{T} \backslash U)$ donde concluímos que $\Omega^{s} \backslash U$ é um aberto em $\mathcal{T}$ e logo em $\Omega^{s}$.

Por outro lado, suponha que existe $b_{j} \not \equiv 0$ que não muda de sinal. Vamos considerar que $b_{j}\left(t_{j}\right) \leq 0$ para todo $t_{j} \in \mathbb{R}$, assim $B_{j}$ é uma função não-crescente em $\mathbb{R}$. Caso $b_{j}\left(t_{j}\right) \geq 0$ para todo $t_{j} \in \mathbb{R}$, os argumentos são semelhantes aos usados a seguir.

Sejam $s \in \mathbb{R}$ e $p=\left(p_{1}, \ldots, p_{n}\right), q=\left(q_{1}, \ldots, q_{n}\right) \in \Omega_{s}$. Vamos assumir que $p_{j} \leq q_{j}$. Para cada $k=1, \ldots, n, k \neq j$, existe um caminho $\sigma_{k}$ (em $\mathbb{R}$ ou $\mathbb{S}^{1}$ ) conectando $q_{k}$ e $p_{k}$. Denotamos por $M$ o maior $M_{k} \doteq \max \left\{B_{k}(\tau) ; \tau \in \sigma_{k}\right\}$.

Seja $C>0$ uma constante tal que $(n-1) M+B_{j}\left(q_{j}+C\right)<s$. Como $B$ é da forma $B(t)=\sum_{k=1}^{n} B_{k}\left(t_{k}\right)$ e $B_{j}$ é uma função não-crescente, existe um caminho $\gamma_{1}$ contido no subnível $\Omega_{s}$ conectando $q$ and $\left(q_{1}, \ldots, q_{j}+C, \ldots, q_{n}\right)$. Definimos agora o caminho $\gamma_{2} \doteq\left(\sigma_{1}, \ldots, q_{j}+C, \ldots, \sigma_{n}\right), \operatorname{logo} B\left(\gamma_{2}(\xi)\right) \leq(n-1) M+B_{j}\left(q_{j}+C\right)<$ $s, \xi \in \mathbb{R}$. Portanto, $\gamma_{2}$ é um caminho contido em $\Omega_{s}$ conectando $\left(q_{1}, \ldots, q_{j}+C, \ldots, q_{n}\right)$ e $\left(p_{1}, \ldots, q_{j}+C, \ldots, p_{n}\right)$.

Finalmente, uma vez que $p \in \Omega_{s}$ e $p_{j} \leq q_{j}+C$, existe um caminho $\gamma_{3}$ contido em $\Omega_{s}$ conectando $p$ e $\left(p_{1}, \ldots, q_{j}+C, \ldots, p_{n}\right)$. Assim, concluímos que o subnível $\Omega_{s}$ é conexo por caminhos. Usando ideias semelhantes às anteriores é possível concluir que o supernível $\Omega^{s}$ também é conexo por caminhos.

Como discutido anteriorente, se $J=\{1, \ldots, n\}$, então o Teorema 2.1 é válido pelo trabalho $[\mathrm{BP}]$. Assim, para que o Teorema 2.1 fique demonstrado, resta verificar os outros dois casos, ou seja, $J=\emptyset$ e $\emptyset \neq J \neq\{1, \ldots, n\}$. Usando o Lema 2.2, percebe-se que esses dois casos do Teorema 2.1 são exatamente as seguintes proposições: 
Proposição 2.3. Se $J=\emptyset$ então o operador $\mathbb{L}$ é globalmente resolúvel se, e somente se, ocorre alguma das alternativas a seguir:

(i) Existe uma função $b_{j}$ que não muda de sinal;

(ii) $a_{0} \in \mathbb{Z}^{n}$, b é exata e os subníveis $\Omega_{s}=\left\{t \in \mathbb{T}^{n} ; B(t)<s\right\}$ e superniveis $\Omega^{s}=\left\{t \in \mathbb{T}^{n} ; B(t)>s\right\}$ são conexos para todo $s \in \mathbb{R}$.

Proposição 2.4. Se $\emptyset \neq J \neq\{1, \ldots, n\}$ então $\mathbb{L}$ é globalmente resolúvel se, e somente se, ocorre alguma das alternativas a seguir:

(i) Existe uma função $b_{j} \not \equiv 0$ que não muda de sinal;

(ii) $\left(a_{j_{1} 0}, \ldots, a_{j_{m} 0}\right) \notin \mathbb{Q}^{m}$ é não-Liouville;

(iii) $a_{0} \in \mathbb{Q}^{n}, q_{J}=q_{*}$, b é exata e os subníveis $\Omega_{s}=\left\{t \in \mathbb{T}^{n} ; B(t)<s\right\}$ e superniveis $\Omega^{s}=\left\{t \in \mathbb{T}^{n} ; B(t)>s\right\}$ são conexos para todo $s \in \mathbb{R}$.

Exemplo 2.5. Como consequência da Proposição 2.4 obtemos um interessante exemplo.

Como $B\left(t_{1}, t_{2}\right)=-\cos t_{2}$ possui apenas subniveis e superniveis conexos em $\mathbb{T}^{2}$, o sistema

$$
\left\{\begin{array}{l}
L_{1}=\frac{\partial}{\partial t_{1}}+\frac{1}{4} \frac{\partial}{\partial x} \\
L_{2}=\frac{\partial}{\partial t_{2}}+\left(\frac{1}{2}+i \operatorname{sen} t_{2}\right) \frac{\partial}{\partial x}
\end{array}\right.
$$

é globalmente resolúvel em $\mathbb{T}^{3}$ uma vez que $q_{J}=q_{*}=4$, enquanto

$$
\left\{\begin{array}{l}
L_{1}=\frac{\partial}{\partial t_{1}}+\frac{1}{2} \frac{\partial}{\partial x} \\
L_{2}=\frac{\partial}{\partial t_{2}}+\left(\frac{1}{4}+i \operatorname{sen} t_{2}\right) \frac{\partial}{\partial x}
\end{array}\right.
$$

não é globalmente resolúvel, pois nesse caso $q_{J}=2<4=q_{*}$.

De modo a simplificar a demonstração das Proposições 2.3 e 2.4, mostraremos que para efeito da resolubilidade global, o operador $\mathbb{L}$ definido em (2.1) pode ser considerado com funções $a_{j}$ constantes, mais precisamente:

$O$ operador $\mathbb{L}=d_{t}+(a(t)+i b(t)) \wedge \frac{\partial}{\partial x}$ é globalmente resolúvel em $\mathbb{T}^{n+1}$ se, e somente se, o operador

$$
d_{t}+\left(a_{0}+i b(t)\right) \wedge \frac{\partial}{\partial x}
$$

é globalmente resolúvel em $\mathbb{T}^{n+1}$, sendo $a_{0}=\sum_{j=1}^{n} a_{j 0} d t_{j}$ e $a_{j 0}$ a média da função $a_{j}$.

De fato, começamos escrevendo a 1-forma real $a$ no seguinte formato

$$
a=a_{0}+d_{t} A
$$


no qual $A \in C^{\infty}\left(\mathbb{T}^{n} ; \mathbb{R}\right)$ e definimos

$$
\begin{aligned}
S: \mathcal{D}^{\prime}\left(\mathbb{T}^{n+1}\right) & \longrightarrow \mathcal{D}^{\prime}\left(\mathbb{T}^{n+1}\right) \\
\sum_{\xi \in \mathbb{Z}} \hat{u}(t, \xi) e^{i \xi x} & \longmapsto \sum_{\xi \in \mathbb{Z}} \hat{u}(t, \xi) e^{i \xi A(t)} e^{i \xi x} .
\end{aligned}
$$

Assim, a aplicação $S$ é um automorfismo de $\mathcal{D}^{\prime}\left(\mathbb{T}^{n+1}\right)$ e de $C^{\infty}\left(\mathbb{T}^{n+1}\right)$. Além disso, a seguinte igualdade ocorre

$$
S \mathbb{L} S^{-1}=d_{t}+\left(a_{0}+i b(t)\right) \wedge \frac{\partial}{\partial x},
$$

o que assegura a afirmação feita anteriormente.

Para simplificar as notações, a partir de agora até o fim do capítulo iremos denotar por $\mathbb{L}$ o operador definido em (2.2).

O novo operador $\mathbb{L}=d_{t}+\left(a_{0}+i b(t)\right) \wedge \frac{\partial}{\partial x}$ está associado ao seguinte sistema de campos vetoriais complexos

$$
L_{j}=\frac{\partial}{\partial t_{j}}+\left(a_{j 0}+i b_{j}\left(t_{j}\right)\right) \frac{\partial}{\partial x}, \quad j=1, \ldots, n .
$$

Observe que em cada campo $L_{j}$ do sistema (2.3) o coeficiente da derivada em $x$ depende apenas da variável $t_{j}$ correspondente. Isso motiva o título dado ao capítulo.

\subsection{Proposição 2.3: demonstração da suficiência}

\subsubsection{Condição suficiente $(i)$}

Na Proposição 2.3 estamos supondo que $J=\emptyset$, ou seja, todas as funções $b_{k}$ são não identicamente nulas. Nesta primeira parte da demonstração da suficiência vamos supor que existe $j$ tal que a função $b_{j}$ não muda de sinal.

Podemos assumir que $b_{j}\left(t_{j}\right) \leq 0$ para todo $t_{j}$, caso contrário basta usar o difeomorfismo $(t, x) \mapsto(t,-x)$ para obter novas coordenadas em $\mathbb{T}^{n+1}$ onde esta propriedade é válida. Portanto, a primitiva $B_{j}$ é uma função monótona não-crescente e $b_{j 0}<0$.

Seja $f=\sum_{k=1}^{n} f_{k}(t, x) d t_{k} \in \mathbb{E}$. Nos cálculos feitos no decorrer desta demonstração usamos série parcial de Fourier na variável $x$. Escrevemos então as séries formais de $u$ e $f_{k}, k=1, \ldots, n$, do seguinte modo

$$
u(t, x)=\sum_{\xi \in \mathbb{Z}} \hat{u}(t, \xi) e^{i \xi x}
$$

e

$$
f_{k}(t, x)=\sum_{\xi \in \mathbb{Z}} \hat{f}_{k}(t, \xi) e^{i \xi x}, \quad k=1, \ldots, n
$$


Substituindo as séries (2.4) e (2.5) na equação $L_{j} u=f_{j}$, sendo $L_{j}$ o campo dado em (2.3), obtemos para cada $\xi \in \mathbb{Z}$ a seguinte equação

$$
\frac{\partial}{\partial t_{j}} \hat{u}(t, \xi)+i \xi\left(a_{j 0}+i b_{j}\left(t_{j}\right)\right) \hat{u}(t, \xi)=\hat{f}_{j}(t, \xi) .
$$

Como $b_{j 0} \neq 0$, para cada $\xi \neq 0$ a equação anterior possui uma única solução dada por

$$
\begin{aligned}
& \hat{u}(t, \xi)=d_{j \xi} \int_{0}^{t_{j}} e^{i \xi\left(\mathcal{C}_{j}\left(s_{j}\right)-\mathcal{C}_{j}\left(t_{j}\right)\right)} \hat{f}_{j}\left(t_{1}, \ldots, s_{j}, \ldots, t_{n}, \xi\right) d s_{j}+ \\
& \quad+\left(d_{j \xi}-1\right) \int_{t_{j}}^{2 \pi} e^{i \xi\left(\mathcal{C}_{j}\left(s_{j}\right)-\mathcal{C}_{j}\left(t_{j}\right)\right)} \hat{f}_{j}\left(t_{1}, \ldots, s_{j}, \ldots, t_{n}, \xi\right) d s_{j}
\end{aligned}
$$

na qual

$$
d_{j \xi}=\left(1-e^{-i 2 \pi \xi c_{j 0}}\right)^{-1} \quad \text { e } \quad \mathcal{C}_{j}\left(t_{j}\right)=a_{j 0} t_{j}+i B_{j}\left(t_{j}\right)
$$

Para $\xi=0$, uma solução da equação

$$
\frac{\partial}{\partial t_{j}} \hat{u}(t, 0)=\hat{f}_{j}(t, 0)
$$

é dada por

$$
\hat{u}(t, 0)=\int_{0}^{t_{j}} \hat{f}_{j}\left(t_{1}, \ldots, s_{j}, \ldots, t_{n}, 0\right) d s_{j}
$$

a qual é bem definida uma vez que $\hat{f}(t, 0)$ é exata (veja a definição de $\mathbb{E}$ em (1.5)).

Antes de continuar a demonstração, provamos o seguinte resultado que será muito útil no decorrer dos cálculos.

Lema 2.6. Sejam $b_{j 0}<0$ e $d_{j \xi} \doteq\left(1-e^{-i 2 \pi \xi\left(a_{j 0}+i b_{j 0}\right)}\right)^{-1}, \xi \in \mathbb{Z}^{*}$. Então, existe uma constante $C>0$ tal que

$$
C^{-1} \leq\left|d_{j \xi}\right| \leq C, \quad \forall \xi \geq 1 \quad \text { e } \quad C^{-1} \leq\left|d_{j \xi}-1\right| \leq C, \quad \forall \xi \leq-1
$$

Demonstração. Consideramos primeiramente $\xi \geq 1$, assim

$$
\left|1-e^{-i 2 \pi \xi\left(a_{j 0}+i b_{j 0}\right)}\right| \leq 1+e^{2 \pi \xi b_{j 0}}<\sum_{k=0}^{\infty}\left(e^{2 \pi b_{j 0}}\right)^{k}=\frac{1}{1-e^{2 \pi b_{j 0}}} \doteq C, \quad \forall \xi \geq 1 .
$$

Por outro lado,

$\left|1-e^{-i 2 \pi \xi\left(a_{j 0}+i b_{j 0}\right)}\right| \geq|1-| e^{-i 2 \pi \xi\left(a_{j 0}+i b_{j 0}\right)}||=1-e^{2 \pi \xi b_{j 0}} \geq 1-e^{2 \pi b_{j 0}}=C^{-1}, \quad \forall \xi \geq 1$. 
Agora para $\xi \leq-1$. Como $d_{j \xi}-1=\left(e^{i 2 \pi \xi\left(a_{j 0}+i b_{j 0}\right)}-1\right)^{-1}$, temos

$$
\left|e^{i 2 \pi \xi\left(a_{j 0}+i b_{j 0}\right)}-1\right| \leq e^{-2 \pi \xi b_{j 0}}+1 \leq \sum_{k=0}^{\infty}\left(e^{2 \pi b_{j 0}}\right)^{k}=C, \quad \forall \xi \leq-1,
$$

além disso,

$$
\left|e^{i 2 \pi \xi\left(a_{j 0}+i b_{j 0}\right)}-1\right| \geq 1-e^{-2 \pi \xi b_{j 0}} \geq 1-e^{2 \pi b_{j 0}}=C^{-1}, \quad \forall \xi \leq-1 .
$$

Vamos mostrar que $u$ definida por (2.4) é uma função suave, sendo $\hat{u}(t, \xi)$ os coeficientes dados pelas fórmulas (2.6) e (2.7), e além disso, $u$ é uma solução da equação $\mathbb{L} u=f$. Com efeito, reescrevemos (2.6) na seguinte forma

$$
\begin{array}{r}
\hat{u}(t, \xi)=d_{j \xi} \int_{0}^{t_{j}} e^{i \xi a_{j 0}\left(s_{j}-t j\right)} e^{\xi\left(B_{j}\left(t_{j}\right)-B_{j}\left(s_{j}\right)\right)} \hat{f}_{j}\left(t_{1}, \ldots, s_{j}, \ldots, t_{n}, \xi\right) d s_{j}+ \\
+\underbrace{e^{i 2 \pi \xi c_{j 0}}\left(d_{j \xi}-1\right)}_{d_{j \xi}} \int_{t_{j}}^{2 \pi} e^{i \xi a_{j 0}\left(s_{j}-t j-2 \pi\right)} e^{\xi\left(B_{j}\left(t_{j}\right)-B_{j}\left(s_{j}\right)+2 \pi b_{j 0}\right)} \hat{f}_{j}\left(t_{1}, \ldots, s_{j}, \ldots, t_{n}, \xi\right) d s_{j} .
\end{array}
$$

Note que na primeira integral $0 \leq s_{j} \leq t_{j}$, logo obtemos $\xi\left(B_{j}\left(t_{j}\right)-B_{j}\left(s_{j}\right)\right) \leq 0$, para todo $\xi \geq 1$. Na segunda integral $t_{j} \leq s_{j} \leq 2 \pi$, portanto

$$
B_{j}\left(t_{j}\right)-B_{j}\left(s_{j}\right)+2 \pi b_{j 0}=-\int_{t_{j}}^{s_{j}} b_{j}\left(\tau_{j}\right) d \tau_{j}+2 \pi b_{j 0} \leq 0,
$$

o que implica $\xi\left(B_{j}\left(t_{j}\right)-B_{j}\left(s_{j}\right)+2 \pi b_{j 0}\right) \leq 0$, para todo $\xi \geq 1$.

Obtemos dessa forma a seguinte desigualdade

$$
|\hat{u}(t, \xi)| \leq 2 \pi C \max _{s \in \mathbb{T}^{n}}\left|\hat{f}_{j}(s, \xi)\right|, \forall t \in \mathbb{T}^{n}, \forall \xi \geq 1
$$

sendo $C>0$ a constante do Lema 2.6.

Reescrevemos agora (2.6) como segue

$$
\begin{aligned}
\hat{u}(t, \xi)=\underbrace{e^{-i 2 \pi \xi c_{j 0}} d_{j \xi}}_{d j \xi-1} & \int_{0}^{t_{j}} e^{i \xi a_{j 0}\left(s_{j}-t j+2 \pi\right)} e^{\xi\left(B_{j}\left(t_{j}\right)-B_{j}\left(s_{j}\right)-2 \pi b_{j 0}\right)} \hat{f}_{j}\left(t_{1}, \ldots, s_{j}, \ldots, t_{n}, \xi\right) d s_{j}+ \\
& +\left(d_{j \xi}-1\right) \int_{t_{j}}^{2 \pi} e^{i \xi a_{j 0}\left(s_{j}-t j\right)} e^{\xi\left(B_{j}\left(t_{j}\right)-B_{j}\left(s_{j}\right)\right)} \hat{f}_{j}\left(t_{1}, \ldots, s_{j}, \ldots, t_{n}, \xi\right) d s_{j} .
\end{aligned}
$$

Na primeira integral $0 \leq s_{j} \leq t_{j}$, logo

$$
B_{j}\left(t_{j}\right)-B_{j}\left(s_{j}\right)-2 \pi b_{j 0}=\int_{s_{j}}^{t_{j}} b_{j}\left(\tau_{j}\right) d \tau_{j}-2 \pi b_{j 0} \geq 0
$$


o que implica $\xi\left(B_{j}\left(t_{j}\right)-B_{j}\left(s_{j}\right)-2 \pi b_{j 0}\right) \leq 0$, para todo $\xi \leq-1$.

Na segunda integral $t_{j} \leq s_{j} \leq 2 \pi$, donde concluímos que $\xi\left(B_{j}\left(t_{j}\right)-B_{j}\left(s_{j}\right)\right) \leq 0$, para todo $\xi \leq-1$. Portanto, obtemos a desigualdade

$$
|\hat{u}(t, \xi)| \leq 2 \pi C \max _{s \in \mathbb{T}^{n}}\left|\hat{f}_{j}(s, \xi)\right|, \forall t \in \mathbb{T}^{n}, \forall \xi \leq-1
$$

na qual $C>0$ é a constante do Lema 2.6.

Para $\xi=0$ temos $|\hat{u}(t, 0)| \leq 2 \pi C \max _{s \in \mathbb{T}^{n}}\left|\hat{f}_{j}(s, 0)\right|$ por $(2.7)$. Assim, provamos até este momento a seguinte estimativa

$$
|\hat{u}(t, \xi)| \leq 2 \pi C \max _{s \in \mathbb{T}^{n}}\left|\hat{f}_{j}(s, \xi)\right|, \forall t \in \mathbb{T}^{n}, \forall \xi \in \mathbb{Z}
$$

Faremos agora estimativas para as derivadas de $\hat{u}(\cdot, \xi), \xi \in \mathbb{Z}$.

Dado $\alpha=\left(\alpha_{1}, \ldots, \alpha_{n}\right) \in \mathbb{Z}_{+}^{n}$ considere o operador diferencial

$$
\partial^{\alpha}=\frac{\partial^{|\alpha|}}{\partial t_{1}^{\alpha_{1}} \ldots \partial t_{n}^{\alpha_{n}}}
$$

no qual $|\alpha|=\alpha_{1}+\cdots+\alpha_{n}$. Assim, $\left|\partial^{\alpha} \hat{u}(t, 0)\right| \leq 2 \pi\left|\partial^{\alpha} \hat{f}_{j}(t, 0)\right|$ se $\alpha_{j}=0$ e $\partial^{\alpha} \hat{u}(t, 0)=$ $\partial^{\alpha-e_{j}} \hat{f}_{j}(t, 0)$ se $\alpha_{j} \geq 1$, sendo $e_{j}=(0, \ldots, 1, \ldots, 0)$ com 1 na $j$-ésima posição.

Sejam $\xi \in \mathbb{Z}^{*}$ e $\beta=\alpha-\alpha_{j} e_{j}$ então

$$
\begin{aligned}
\partial^{\beta} \hat{u}(t, \xi) & =d_{j \xi} \int_{0}^{t_{j}} e^{i \xi\left(\mathcal{C}_{j}\left(s_{j}\right)-\mathcal{C}_{j}\left(t_{j}\right)\right)} \partial^{\beta} \hat{f}_{j}\left(t_{1}, \ldots, s_{j}, \ldots, t_{n}, \xi\right) d s_{j}+ \\
& +\left(d_{j \xi}-1\right) \int_{t_{j}}^{2 \pi} e^{i \xi\left(\mathcal{C}_{j}\left(s_{j}\right)-\mathcal{C}_{j}\left(t_{j}\right)\right)} \partial^{\beta} \hat{f}_{j}\left(t_{1}, \ldots, s_{j}, \ldots, t_{n}, \xi\right) d s_{j} .
\end{aligned}
$$

Note que, como antes vale a desigualdade

$$
\left|\partial^{\beta} \hat{u}(t, \xi)\right| \leq 2 \pi C \max _{s \in \mathbb{T}^{n}}\left|\partial^{\beta} \hat{f}_{j}(s, \xi)\right|, \forall t \in \mathbb{T}^{n}, \forall \xi \in \mathbb{Z}^{*} .
$$

Vamos calcular agora as derivadas de $\partial^{\beta} \hat{u}(\cdot, \xi), \xi \in \mathbb{Z}^{*}$, em relação a variável $t_{j}$.

$$
\begin{gathered}
\frac{\partial}{\partial t_{j}} \partial^{\beta} \hat{u}(t, \xi)=-i \xi c_{j}\left(t_{j}\right) \partial^{\beta} \hat{u}(t, \xi)+\partial^{\beta} \hat{f}_{j}(t, \xi), \\
\frac{\partial^{2}}{\partial t_{j}^{2}} \partial^{\beta} \hat{u}(t, \xi)=\left[-i^{2} \xi^{2} c_{j}^{2}\left(t_{j}\right)-i \xi \frac{\partial c_{j}}{\partial t_{j}}\left(t_{j}\right)\right] \partial^{\beta} \hat{u}(t, \xi)+i \xi c_{j}\left(t_{j}\right) \partial^{\beta} \hat{f}_{j}(t, \xi)+\frac{\partial}{\partial t_{j}} \partial^{\beta} \hat{f}_{j}(t, \xi),
\end{gathered}
$$

sendo $c_{j}\left(t_{j}\right)=a_{j 0}+i b_{j}\left(t_{j}\right)$. Portanto, para $m \in \mathbb{N}$ obtemos a seguinte igualdade

$$
\frac{\partial^{m}}{\partial t_{j}^{m}} \partial^{\beta} \hat{u}(t, \xi)=P\left(t_{j}, \xi\right) \partial^{\beta} \hat{u}(t, \xi)+Q(t, \xi), \quad \xi \in \mathbb{Z}^{*}
$$


na qual $P$ é um polinômio que depende das derivadas de $c_{j}$ (de ordem até $m$ ) e de potências de $\xi$ com grau no máximo $m$. $Q$ é outro polinômio em $\xi$ de grau menor que $m$, cujos coeficientes envolvem derivadas de $c_{j}$ e $\partial^{\beta} \hat{f}_{j}(t, \xi)$. Como $c_{j}$ e suas derivadas de ordem até $m$ são limitadas em $\mathbb{T}^{1}$, existe uma constante $C_{m}>0$ tal que

$$
\left|P\left(t_{j}, \xi\right)\right| \leq C_{m}|\xi|^{m} \text { e }|Q(t, \xi)| \leq C_{m}|\xi|^{m} \sum_{k=0}^{m-1}\left|\frac{\partial^{k}}{\partial t_{j}^{k}} \partial^{\beta} \hat{f}_{j}(t, \xi)\right|, \forall t \in \mathbb{T}^{n} .
$$

Se $m=\alpha_{j}$, então da igualdade (2.8) segue

$$
\begin{aligned}
\left|\partial^{\alpha} \hat{u}(t, \xi)\right| & \leq C_{\alpha_{j}}|\xi|^{\alpha_{j}}\left(\left|\partial^{\beta} \hat{u}(t, \xi)\right|+\sum_{k=0}^{\alpha_{j}-1}\left|\frac{\partial^{k}}{\partial t_{j}^{k}} \partial^{\beta} \hat{f}_{j}(t, \xi)\right|\right) \\
& \leq \tilde{C}_{\alpha_{j}}|\xi|^{\alpha_{j}} \sum_{k=0}^{\alpha_{j}-1} \max _{s \in \mathbb{T}^{n}}\left|\frac{\partial^{k}}{\partial t_{j}^{k}} \partial^{\beta} \hat{f}_{j}(s, \xi)\right|, \forall t \in \mathbb{T}^{n}, \forall \xi \in \mathbb{Z}^{*},
\end{aligned}
$$

e se $\alpha_{j}=0$ temos

$$
\left|\partial^{\alpha} \hat{u}(t, \xi)\right| \leq 2 \pi C \max _{s \in \mathbb{T}^{n}}\left|\partial^{\alpha} \hat{f}_{j}(s, \xi)\right|, \forall t \in \mathbb{T}^{n}, \forall \xi \in \mathbb{Z}^{*}
$$

Uma vez que a função $f_{j}$ é suave, as desigualdades anteriores implicam que $u \in$ $C^{\infty}\left(\mathbb{T}^{n+1}\right)$. Nosso próximo passo é mostrar que $u$ é solução global do sistema $L_{k} u=f_{k}$, $k=1, \ldots, n$. Em vista da construção da função $u$ temos $L_{j} u=f_{j}$, logo resta provar que $L_{k} u=f_{k}$ para $k \neq j$ :

$$
\begin{aligned}
& \widehat{L_{k} u}(t, \xi)=\frac{\partial \hat{u}}{\partial t_{k}}(t, \xi)+i \xi c_{k}\left(t_{k}\right) \hat{u}(t, \xi) \\
= & d_{j \xi} \int_{0}^{t_{j}} e^{i \xi\left(\mathcal{C}_{j}\left(s_{j}\right)-\mathcal{C}_{j}\left(t_{j}\right)\right)} \widehat{L_{k} f_{j}}\left(t_{1}, \ldots, s_{j}, \ldots, t_{n}, \xi\right)+ \\
+ & \left.\left(d_{j \xi}-1\right) \int_{t_{j}}^{2 \pi} e^{i \xi\left(\mathcal{C}_{j}\left(s_{j}\right)-\mathcal{C}_{j}\left(t_{j}\right)\right)} \widehat{L_{k} f_{j}}\left(t_{1}, \ldots, s_{j}, \ldots, t_{n}, \xi\right)\right) d s_{j} \\
= & d_{j \xi} \int_{0}^{t_{j}} e^{i \xi\left(\mathcal{C}_{j}\left(s_{j}\right)-\mathcal{C}_{j}\left(t_{j}\right)\right)} \widehat{L_{j} f_{k}}\left(t_{1}, \ldots, s_{j}, \ldots, t_{n}, \xi\right)+ \\
+ & \left.\left(d_{j \xi}-1\right) \int_{t_{j}}^{2 \pi} e^{i \xi\left(\mathcal{C}_{j}\left(s_{j}\right)-\mathcal{C}_{j}\left(t_{j}\right)\right)} \widehat{L_{j} f_{k}}\left(t_{1}, \ldots, s_{j}, \ldots, t_{n}, \xi\right)\right) d s_{j} \\
= & d_{j \xi} e^{-i \xi \mathcal{C}_{j}\left(t_{j}\right)} \int_{0}^{t_{j}} \frac{\partial}{\partial s_{j}}\left(e^{i \xi \mathcal{C}_{j}\left(s_{j}\right)} \hat{f}_{k}\left(t_{1}, \ldots, s_{j}, \ldots, t_{n}, \xi\right)\right) d s_{j}+ \\
+ & \left(d_{j \xi}-1\right) e^{-i \xi \mathcal{C}_{j}\left(t_{j}\right)} \int_{t_{j}}^{2 \pi} \frac{\partial}{\partial s_{j}}\left(e^{i \xi \mathcal{C}_{j}\left(s_{j}\right)} \hat{f}_{k}\left(t_{1}, \ldots, s_{j}, \ldots, t_{n}, \xi\right)\right) d s_{j} \\
= & d_{j \xi}\left(\hat{f}_{k}(t, \xi)-e^{-i \xi \mathcal{C}_{j}\left(t_{j}\right)} e^{i \xi \mathcal{C}_{j}(0)} \hat{f}_{k}\left(t_{1}, \ldots, 0, \ldots, t_{n}, \xi\right)\right)+ \\
+ & \left(d_{j \xi}-1\right)\left(e^{-i \xi \mathcal{C}_{j}\left(t_{j}\right)} e^{i \xi \mathcal{C}_{j}(2 \pi)} \hat{f}_{k}\left(t_{1}, \ldots, 2 \pi, \ldots, t_{n}, \xi\right)-\hat{f}_{k}(t, \xi)\right) \\
= & \hat{f}_{k}(t, \xi),
\end{aligned}
$$


pois $\left(d_{j \xi}-1\right) e^{i \xi \mathcal{C}_{j}(2 \pi)}=d_{j \xi} e^{i \xi \mathcal{C}_{j}(0)}$ e $\hat{f}_{k}(\cdot, \xi)$ é $2 \pi$-periódica.

\subsubsection{Condição suficiente $(i i)$}

Lembremos que as hipóteses da condição (ii) são: $b$ é exata (logo possui uma primitiva global $B$ definida em $\left.\mathbb{T}^{n}\right), a_{0} \in \mathbb{Z}^{n}$ e os conjuntos de subnível $\Omega_{s}=\left\{t \in \mathbb{T}^{n} ; B(t)<\right.$ $s\}$ bem como os de supernível $\Omega^{s}=\left\{t \in \mathbb{T}^{n} ; B(t)>s\right\}$ são conexos para todo $s \in \mathbb{R}$.

Considere a seguinte aplicação

$$
\begin{aligned}
T: \mathcal{D}^{\prime}\left(\mathbb{T}^{n+1}\right) & \longrightarrow \mathcal{D}^{\prime}\left(\mathbb{T}^{n+1}\right) \\
\sum_{\xi \in \mathbb{Z}} \hat{u}(t, \xi) e^{i \xi x} & \longmapsto \sum_{\xi \in \mathbb{Z}} \hat{u}(t, \xi) e^{-i \xi a_{0} \cdot t} e^{i \xi x} .
\end{aligned}
$$

Veja que $T$ fica bem definida uma vez que $a_{0} \in \mathbb{Z}^{n}$. Além disso, a aplicação $T$ define um automorfismo de $\mathcal{D}^{\prime}\left(\mathbb{T}^{n+1}\right)$ (e de $\left.C^{\infty}\left(\mathbb{T}^{n+1}\right)\right)$ e obtemos a seguinte identidade

$$
T^{-1} \mathbb{L} T=\mathbb{L}_{0} \doteq d_{t}+i b(t) \wedge \frac{\partial}{\partial x} .
$$

Conforme o trabalho $[\mathrm{CH}]$, o operador $\mathbb{L}_{0}$ é globalmente resolúvel se, e somente se, todos os subníveis e superníveis de $B$ são conexos em $\mathbb{T}^{n}$, visto que a 1-forma $b$ é exata. Portanto, por hipótese temos $\mathbb{L}_{0}$ globalmente resolúvel e da identidade $T^{-1} \mathbb{L} T=\mathbb{L}_{0}$ concluímos que $\mathbb{L}$ também é globalmente resolúvel.

\subsection{Proposição 2.3: demonstração da necessidade}

Como o título sugere, o objetivo desta seção será mostrar que para $\mathbb{L}$ ser globalmente resolúvel é necessário que alguma das condições $(i)$ ou (ii) apresentadas na Proposição 2.3 esteja cumprida. Para isso vamos supor que $(i)$ e $(i i)$ não são válidas, ou seja, daqui em diante todas as funções $b_{j}$ (as quais por hipótese não são identicamente nulas) mudam de sinal e alguma das seguintes condições não está satisfeita: $b$ é exata, $a_{0} \in \mathbb{Z}^{n}$ e todos os subníveis e superníveis de uma primitiva $B$ da 1 -forma $b$ são conexos em $\mathbb{T}^{n}$.

Dando continuidade à demonstração, suponha que $b$ é exata, $a_{0} \in \mathbb{Z}^{n}$ e uma primitiva global $B$ de $b$ possui um subnível ou um supernível desconexo em $\mathbb{T}^{n}$. Então, o operador $\mathbb{L}_{0}=d_{t}+i b(t) \wedge \frac{\partial}{\partial x}$ não é globalmente resolúvel por $[\mathrm{CH}]$, o que implica a não resolubilidade global de $\mathbb{L}$ por (2.9).

Suponha agora que $b$ não é exata ou $a_{0} \notin \mathbb{Z}^{n}$ o que equivale a supor que $c_{0}=$ $a_{0}+i b_{0}=\left(c_{10}, \ldots, c_{n 0}\right) \notin \mathbb{Z}^{n}$. Consideramos dois casos, a saber: 
- Caso 1: $\left\{c_{10}, \ldots, c_{n 0}\right\} \cap \mathbb{Z}=\emptyset$;

- Caso 2: $\left\{c_{10}, \ldots, c_{n 0}\right\} \cap \mathbb{Z} \neq \emptyset$.

Passamos agora à demonstração da não resolubilidade de $\mathbb{L}$ no caso 1 , a qual será dada pelas Proposições 2.8 e 2.9 .

$$
\text { Caso 1: }\left\{c_{10}, \ldots, c_{n 0}\right\} \cap \mathbb{Z}=\emptyset
$$

Seja $B=\sum_{j=1}^{n} B_{j}\left(t_{j}\right)$ uma primitiva do pull-back $\Pi^{*} b$ através do recobrimento universal $\Pi: \mathbb{R}^{n} \rightarrow \mathbb{T}^{n}$. Uma vez que cada função $b_{j}$ muda de sinal, cada função $B_{j}$ possui extremos locais (pelo menos dois em cada intervalo de comprimento $2 \pi$, visto que $b_{j}$ é periódica). Portanto, podemos assumir (fazendo uma translação) que o máximo de $B$ sobre o cubo $[0,2 \pi]^{n}$ não ocorre na fronteira. Além disso, pelo Lema 1.4 , sempre que $b_{j 0} \neq 0$ podemos assumir que $b_{j 0}<0$. Assim, daqui em diante vamos considerar as seguintes condições:

$\star b_{j 0} \leq 0$ para todo $j=1, \ldots, n$;

* O máximo de $B$ sobre o cubo $[0,2 \pi]^{n}$ não ocorre na fronteira.

Podemos assumir também que $B(0)=0$, pois caso isso não ocorra basta considerar a primitiva $B-B(0)$.

A ideia natural será construir uma 1-forma $f \in \mathbb{E}$ de modo que não exista solução $u \in \mathcal{D}^{\prime}\left(\mathbb{T}^{n+1}\right)$ da equação $\mathbb{L} u=f$. Para que a 1 -forma $f=f_{1} d t_{1}+\cdots+f_{n} d t_{n}$ pertença a $\mathbb{E}$ ela deve satisfazer $\mathbb{L} f=0$, o que é equivalente a $L_{j} f_{k}-L_{k} f_{j}=0$ para todo $k, j=1, \ldots, n$. Essa condição juntamente com $\left[L_{j}, L_{k}\right]=0, k, j=1, \ldots, n$ implicam a seguinte identidade

$$
L_{n} L_{n-1} \ldots L_{2} L_{1} u=L_{\sigma(n)} L_{\sigma(n-1)} \ldots L_{\sigma(2)} L_{\sigma(1)} u, \quad \forall \sigma \in \mathcal{S}_{n}
$$

sendo $\mathcal{S}_{n}$ o grupo das permutações dos inteiros $1, \ldots, n$. Ou seja, se existe solução $u$ da equação $\mathbb{L} u=f$ então vale a identidade anterior. Nosso plano será utilizar (2.10) para obter as funções $f_{1}, \ldots, f_{n}$.

Motivados por (2.10), substituímos as séries formais

$$
u(t, x)=\sum_{\xi \in \mathbb{Z}} \hat{u}(t, \xi) e^{i \xi x} \quad \text { e } \quad h(t, x)=\sum_{\xi \in \mathbb{Z}} \hat{h}(t, \xi) e^{i \xi x}
$$

na equação

$$
h=L_{n} L_{n-1} \ldots L_{1} u
$$


sendo a função $h$ escolhida posteriormente. Obtemos assim para cada $\xi \in \mathbb{Z}$ a equação

$$
\hat{h}(t, \xi)=\left(\frac{\partial}{\partial t_{n}}+i \xi c_{n}\left(t_{n}\right)\right) \cdots\left(\frac{\partial}{\partial t_{1}}+i \xi c_{1}\left(t_{1}\right)\right) \hat{u}(t, \xi) .
$$

Considere agora o conjunto $\mathcal{F}=\left\{c_{10}, \ldots, c_{n 0}\right\} \cap \mathbb{Q}$. Se $c_{j 0} \in \mathcal{F}$, escrevemos $c_{j 0}=$ $r_{j} / s_{j}$ com $r_{j} \in \mathbb{Z}$ e $s_{j} \in \mathbb{N}$ primos entre si e definimos o conjunto

$$
\mathcal{G}=\bigcup_{c_{j 0} \in \mathcal{F}} s_{j} \mathbb{Z}
$$

Como cada $c_{j 0} \notin \mathbb{Z}$, se $c_{j 0} \in \mathcal{F}$ então $s_{j}>1$, logo $\mathbb{Z}_{ \pm} \backslash \mathcal{G}$ possui infinitos elementos. Para cada $j=1, \ldots, n$ e cada elemento $\xi \in \mathbb{Z} \backslash \mathcal{G}$ a equação

$$
\left(\frac{\partial}{\partial t_{j}}+i \xi c_{j}\left(t_{j}\right)\right) v(t, \xi)=g_{\xi}(t), \quad g_{\xi} \in C^{\infty}\left(\mathbb{T}^{n}\right),
$$

possui uma única solução $v$ dada pela fórmula

$$
v(t, \xi)=d_{j \xi} \int_{0}^{2 \pi} e^{i \xi\left(\mathcal{C}_{j}\left(t_{j}-s_{j}\right)-\mathcal{C}_{j}\left(t_{j}\right)\right)} g_{\xi}\left(t_{1}, \ldots, t_{j}-s_{j}, \ldots, t_{n}\right) d s_{j},
$$

sendo $d_{j \xi}=\left(1-e^{-i 2 \pi \xi c_{j 0}}\right)^{-1}$ e $\mathcal{C}_{j}(t)=a_{j 0} t_{j}+i B_{j}\left(t_{j}\right)$. Consequentemente, para cada $\xi \in \mathbb{Z} \backslash \mathcal{G}$ a equação (2.11) possui uma única solução dada por

$$
\hat{u}(t, \xi)=d_{\xi} \int_{[0,2 \pi]^{n}} e^{i \xi(\mathcal{C}(t-s)-\mathcal{C}(t))} \hat{h}(t-s, \xi) d s,
$$

na qual $d_{\xi}=d_{1 \xi} \ldots d_{n \xi}$ e $\mathcal{C}(t)=a_{0} \cdot t+i B(t)$.

Seja $v(\cdot, \xi)$ a expressão definida pelo lado direito de (2.13), a qual é bem definida pois só depende da escolha da função $h$. Definimos então as funções

$$
f_{j}(t, x)=\sum_{\xi \in \mathbb{Z}_{+} \backslash \mathcal{G}} \hat{f}_{j}(t, \xi) e^{i \xi x}, \quad j=1, \ldots, n,
$$

nas quais os elementos $\hat{f}_{j}(\cdot, \xi)$ são dados por

$$
\hat{f}_{j}(t, \xi) \doteq\left(\frac{\partial}{\partial t_{j}}+i \xi c_{j}\left(t_{j}\right)\right) v(t, \xi), \quad j=1, \ldots, n, \quad \xi \in \mathbb{Z}_{+} \backslash \mathcal{G}
$$

Vamos mostrar na Proposição 2.8 (adiante) que para uma certa escolha de $h$ as funções $f_{1}, \ldots, f_{n}$ são suaves. Assim, se $f \doteq \sum_{j=1}^{n} f_{j} d t_{j}$ então a definição das funções $f_{j}$ assegura que a condição de compatibilidade $\mathbb{L} f=0$ estará satisfeita. De fato, basta notar que

$$
\begin{aligned}
{\widehat{L_{k} f}}_{j}(t, \xi) & =\left(\frac{\partial}{\partial t_{k}}+i \xi c_{k}\left(t_{k}\right)\right)\left(\frac{\partial}{\partial t_{j}}+i \xi c_{j}\left(t_{j}\right)\right) v(t, \xi)= \\
& =\left(\frac{\partial}{\partial t_{j}}+i \xi c_{j}\left(t_{j}\right)\right)\left(\frac{\partial}{\partial t_{k}}+i \xi c_{k}\left(t_{k}\right)\right) v(t, \xi)=\widehat{L_{j} f_{k}}(t, \xi), \quad \xi \in \mathbb{Z}_{+} \backslash \mathcal{G},
\end{aligned}
$$


portanto $L_{k} f_{j}=L_{j} f_{k}, k, j=1, \ldots, n$, o que equivale a $\mathbb{L} f=0$.

Além disso, se $\xi \in \mathbb{Z}_{+} \backslash \mathcal{G}$ é tal que $\xi c_{0} \in \mathbb{Z}^{n}$ então

$$
\hat{f}_{j}(t, \xi) e^{i \xi\left(c_{0} \cdot t+C(t)\right)}=\frac{\partial}{\partial t_{j}}\left(v(t, \xi) e^{i \xi\left(c_{0} \cdot t+C(t)\right)}\right), \quad j=1, \ldots, n,
$$

sendo $C(t) \doteq \mathcal{C}(t)-c_{0} \cdot t$ uma função suave e periódica. Portanto a 1-forma

$$
\hat{f}_{1}(t, \xi) e^{i \xi\left(c_{0} \cdot t+C(t)\right)} d t_{1}+\cdots+\hat{f}_{n}(t, \xi) e^{i \xi\left(c_{0} \cdot t+C(t)\right)} d t_{n}
$$

é exata sempre que $\xi \in \mathbb{Z}_{+} \backslash \mathcal{G}$ satisfaz $\xi c_{0} \in \mathbb{Z}^{n}$.

Portanto, ao fazer uma escolha conveniente da função $h$ teremos $f \in \mathbb{E}$ e pela definição de $f$ se existir uma solução $u$ da equação $\mathbb{L} u=f$ então os coeficientes parciais de Fourier $\hat{u}(\cdot, \xi)$ serão os dados em $(2.13)$.

Desenvolvendo $\hat{f}_{j}(\cdot, \xi)$ em $(2.14)$ chegamos à seguinte expressão

$$
\hat{f}_{j}(t, \xi)=\frac{d_{\xi}}{d_{j \xi}} \int_{[0,2 \pi]^{n-1}} e^{i \xi\left(\mathcal{C}\left(t-s+s_{j} e_{j}\right)-\mathcal{C}(t)\right)} \hat{h}\left(t-s+s_{j} e_{j}, \xi\right) d s^{(j)},
$$

na qual $d s^{(j)}=d s_{1} \ldots d s_{j-1} d s_{j+1} \ldots d s_{n}$ e $\left\{e_{1}, \ldots, e_{n}\right\}$ é a base canônica do $\mathbb{R}^{n}$.

\section{Construção da função $h$}

Denotamos por $M$ o máximo da função $B$ sobre o cubo $[0,2 \pi]^{n}$ e por $\widetilde{M}$ o maior $M_{k} \doteq \max \left\{B(t) ; t \in[0,2 \pi]^{n} \cap\left\{t_{k}=0\right\}\right\}, k=1, \ldots, n$, no qual $\left\{t_{k}=0\right\}$ denota o hiperplano $\left\{t=\left(t_{1}, \ldots, t_{n}\right) \in \mathbb{R}^{n} ; t_{k}=0\right\}$. Então, pelas argumentações feitas no início deste caso 1 , temos $0 \leq \widetilde{M}<M$.

Seja $0<\delta<\pi / 2$ constante. Considere uma função de corte $\chi_{\delta} \in C_{c}^{\infty}\left(\mathbb{R}^{n}\right)$ tal que

$$
\chi_{\delta}(t)= \begin{cases}1 & \text { se } t \in Q_{\delta} \\ 0 & \text { se } t \notin Q_{2 \delta}\end{cases}
$$

e $0 \leq \chi_{\delta}(t) \leq 1$, para todo $t \in \mathbb{R}^{n}$, sendo $Q_{\delta}=\left\{t \in \mathbb{R}^{n} ;|t|<\delta\right\}$.

Definimos assim as funções suaves e periódicas

$$
\begin{aligned}
& p(t)=\sum_{\nu \in \mathbb{Z}^{n}} a_{0} \cdot\left(t^{*}-t-2 \pi \nu\right) \chi_{\delta}(t+2 \pi \nu), \quad t \in \mathbb{R}^{n} \quad \mathrm{e} \\
& q(t)=1+\sum_{\nu \in \mathbb{Z}^{n}}\left(|t+2 \pi \nu|^{2}-1\right) \chi_{\delta}(t+2 \pi \nu), \quad t \in \mathbb{R}^{n},
\end{aligned}
$$

sendo $t^{*} \in(0,2 \pi)^{n}$ tal que $B\left(t^{*}\right)=M$.

As funções $p$ e $q$ gozam das seguintes propriedades: 
- $q(t) \geq 0$ para todo $t \in \mathbb{R}^{n}$ e $q(t)=0$ se, e somente se, $t=2 \pi \nu, \nu \in \mathbb{Z}^{n}$;

- Se $|t|<\delta$ então $p(t)=a_{0} \cdot\left(t^{*}-t\right)$ e $q(t)=|t|^{2}$.

Finalmente, definimos a função $h$ por

$$
h(t, x) \doteq \sum_{\xi \in \mathbb{Z}_{+} \backslash \mathcal{G}} \hat{h}(t, \xi) e^{i \xi x},
$$

sendo os coeficientes $\hat{h}(\cdot, \xi)$ dados por

$$
\hat{h}(t, \xi)=\left(d_{\xi}\right)^{-1} e^{-\xi(M+K q(t)-\lambda-i p(t))}, \quad \xi \in \mathbb{Z}_{+} \backslash \mathcal{G},
$$

com $K>0$ e $0<\lambda<(M-\widetilde{M}) / 2$ constantes. A constante $K$ será escolhida suficientemente grande no decorrer do texto.

Proposição 2.7. $h \in C^{\infty}\left(\mathbb{T}^{n+1}\right)$.

Demonstração. Note que $M+K q(t)-\lambda \geq M-\lambda>0$. Então, como existe uma constante $C>0$ tal que $\left|\left(d_{\xi}\right)^{-1}\right| \leq C$ para todo $\xi \in \mathbb{Z}_{+} \backslash \mathcal{G}$ (veja o Lema 2.6), segue a desigualdade

$$
|\hat{h}(t, \xi)| \leq C e^{-\xi(M-\lambda)}, \quad \forall t \in[0,2 \pi]^{n} \text { e } \forall \xi \in \mathbb{Z}_{+} \backslash \mathcal{G} .
$$

Dado $\alpha=\left(\alpha_{1}, \ldots, \alpha_{n}\right) \in \mathbb{Z}_{+}^{n}$, temos a identidade $\partial^{\alpha} \hat{h}(t, \xi)=P(t, \xi) \hat{h}(t, \xi)$ na qual $P(t, \xi)$ é um polinômio envolvendo potências de $\xi$ com grau no máximo $|\alpha|$ e derivadas de $p$ e $q$, as quais são limitadas em $[0,2 \pi]^{n}$. Então, existe uma constante $C_{\alpha}>0$ tal que

$$
|P(t, \xi)| \leq C_{\alpha} \xi^{|\alpha|}, \quad \forall t \in[0,2 \pi]^{n} \text { e } \forall \xi \in \mathbb{Z}_{+} \backslash \mathcal{G}
$$

Portanto,

$$
\left|\partial^{\alpha} \hat{h}(t, \xi)\right| \leq C C_{\alpha} \xi^{|\alpha|} e^{-\xi(M-\lambda)}, \quad \forall t \in[0,2 \pi]^{n} \text { e } \forall \xi \in \mathbb{Z}_{+} \backslash \mathcal{G}
$$

donde concluímos que $h \in C^{\infty}\left(\mathbb{T}^{n+1}\right)$.

Agora, para cada $\xi \in \mathbb{Z}_{+} \backslash \mathcal{G}$, substituindo os coeficientes (2.16) em (2.13) e (2.15), obtemos as seguintes expressões

$$
\hat{u}(t, \xi)=\int_{[0,2 \pi]^{n}} e^{\xi\left(\varphi_{1}(t, s)+i \varphi_{2}(t, s)\right)} d s,
$$

e

$$
\hat{f}_{j}(t, \xi)=\frac{1}{d_{j \xi}} \int_{[0,2 \pi]^{n-1}} e^{\xi\left(\varphi_{1}\left(t, s-s_{j} e_{j}\right)+i \varphi_{2}\left(t, s-s_{j} e_{j}\right)\right)} d s^{(j)}, j=1, \ldots, n,
$$

sendo $\varphi_{1}(t, s) \doteq B(t)-B(t-s)-K q(t-s)-M+\lambda$ e $\varphi_{2}(t, s) \doteq p(t-s)-a_{0} \cdot s$ funções a valores reais. 
Proposição 2.8. Para $K>0$ suficientemente grande, $f_{j} \in C^{\infty}\left(\mathbb{T}^{n+1}\right), j=1, \ldots, n$.

Demonstração. Vamos identificar $s-s_{j} e_{j} \operatorname{com}\left(s_{1}, \ldots, s_{j-1}, s_{j+1}, \ldots, s_{n}\right)$. Iniciemos a demonstração definindo o conjunto

$$
\mathcal{Q}_{j} \doteq\left\{\left(t, s-s_{j} e_{j}\right) \in[0,2 \pi]^{2 n-1} ; q\left(t-s+s_{j} e_{j}\right)=0\right\}
$$

Como $q\left(t-s+s_{j} e_{j}\right)=0$ se, e somente se, $t-s+s_{j} e_{j}=2 \nu \pi, \nu \in \mathbb{Z}^{n}$, obtemos

$$
\begin{aligned}
\varphi_{1}\left(t, s-s_{j} e_{j}\right) & =B(t)-B\left(t-s+s_{j} e_{j}\right)-K q\left(t-s+s_{j} e_{j}\right)-M+\lambda \\
& =B\left(s-s_{j} e_{j}+2 \nu \pi\right)-B(2 \nu \pi)-M+\lambda \\
& =B\left(s-s_{j} e_{j}\right)-M+\lambda \\
& \leq \widetilde{M}-M+\lambda<\frac{\widetilde{M}-M}{2}, \quad\left(t, s-s_{j} e_{j}\right) \in \mathcal{Q}_{j} .
\end{aligned}
$$

O conjunto $\mathcal{Q}_{j}$ é fechado uma vez que a função $[0,2 \pi]^{2 n-1} \ni\left(t, s-s_{j} e_{j}\right) \mapsto q\left(t-s+s_{j} e_{j}\right)$ é contínua. Assim, existe um aberto $U \supset \mathcal{Q}_{j}$ tal que

$$
\varphi_{1}\left(t, s-s_{j} e_{j}\right) \leq \frac{M-\widetilde{M}}{2}, \quad\left(t, s-s_{j} e_{j}\right) \in U .
$$

Considere agora o compacto $[0,2 \pi]^{2 n-1} \backslash U$ e as constantes

$$
\sigma \doteq \min \left\{q\left(t-s+s_{j} e_{j}\right) ;\left(t, s-s_{j} e_{j}\right) \in[0,2 \pi]^{2 n-1} \backslash U\right\}
$$

e

$$
\mu \doteq \min \left\{B\left(t-s+s_{j} e_{j}\right) ;\left(t, s-s_{j} e_{j}\right) \in[0,2 \pi]^{2 n-1} \backslash U\right\} .
$$

Note que $\sigma>0$. Logo, escolhemos $K>0$ de forma que

$$
K \geq \frac{1}{\sigma}\left(-\mu+\lambda+\frac{M-\widetilde{M}}{2}\right)
$$

e obtemos

$$
\begin{aligned}
\varphi_{1}\left(t, s-s_{j} e_{j}\right) & \leq-\mu-K \sigma+\lambda \\
& \leq \frac{\widetilde{M}-M}{2}, \quad\left(t, s-s_{j} e_{j}\right) \in[0,2 \pi]^{2 n-1} \backslash U .
\end{aligned}
$$

Concluímos assim que

$$
\varphi_{1}\left(t, s-s_{j} e_{j}\right) \leq \frac{\widetilde{M}-M}{2}, \quad\left(t, s-s_{j} e_{j}\right) \in[0,2 \pi]^{2 n-1} .
$$

Então por (2.18) chegamos na seguinte desigualdade

$$
\left|\hat{f}_{j}(t, \xi)\right| \leq C e^{-\xi \frac{M-\widetilde{M}}{2}}, \quad \forall \xi \in \mathbb{Z}_{+} \backslash \mathcal{G}
$$


na qual $C>0$ é uma constante que não depende de $t$ e $\xi$.

Seja $\varphi(t, s)=\varphi_{1}(t, s)+i \varphi_{2}(t, s)$. Dado $\alpha \in \mathbb{Z}_{+}^{n}$, novamente por (2.18) obtemos

$$
\partial^{\alpha} \hat{f}_{j}(t, \xi)=\frac{1}{d_{j \xi}} \int_{[0,2 \pi]^{n-1}} P\left(t, s-s_{j} e_{j}, \xi\right) e^{\xi \varphi\left(t, s-s_{j} e_{j}\right)} d s^{(j)},
$$

sendo $P$ um polinômio que envolve potências de $\xi$ com grau no máximo $|\alpha|$ e derivadas de $\varphi$, as quais são limitadas em $[0,2 \pi]^{2 n-1}$. Então, existe uma constante $C_{\alpha}>0$ tal que

$$
\left|P\left(t, s-s_{j} e_{j}, \xi\right)\right| \leq C_{\alpha} \xi^{|\alpha|}, \quad\left(t, s-s_{j} e_{j}\right) \in[0,2 \pi]^{2 n-1},
$$

donde obtemos a desigualdade

$$
\begin{aligned}
\left|\partial^{\alpha} \hat{f}_{j}(t, \xi)\right| & \leq \frac{1}{\left|d_{j \xi}\right|} \int_{[0,2 \pi]^{n-1}}\left|P\left(t, s-s_{j} e_{j}, \xi\right)\right|\left|e^{\xi \varphi\left(t, s-s_{j} e_{j}\right)}\right| d s^{(j)} \\
& \leq C C_{\alpha} \xi^{|\alpha|} e^{-\xi \frac{M-\widetilde{M}}{2}}, \quad \forall \xi \in \mathbb{Z}_{+} \backslash \mathcal{G}
\end{aligned}
$$

concluindo que $f_{j} \in C^{\infty}\left(\mathbb{T}^{n+1}\right)$.

Proposição 2.9. Para $K>0$ suficientemente grande, não existe distribuição $u \in$ $\mathcal{D}^{\prime}\left(\mathbb{T}^{n+1}\right)$ cujos coeficientes parciais de Fourier na variável $x$ sejam os dados por (2.17).

Demonstração. Uma vez que $B(0)=0$, escolhemos $0<\delta_{0}<\delta$ tal que $|B(t)|<\lambda / 2$ para todo $|t|<\delta_{0}$. Para este $\delta_{0}$ escrevemos a igualdade

$$
\hat{u}\left(t^{*}, \xi\right)=I_{\xi}+J_{\xi}
$$

na qual $t^{*} \in(0,2 \pi)^{n}$ satisfaz $B\left(t^{*}\right)=M\left(t^{*}\right.$ é o mesmo ponto que aparecece na construção da função $p$ ) e

$$
\begin{aligned}
& I_{\xi}=\int_{\left|s-t^{*}\right|<\delta_{0}} e^{i \xi\left(p\left(t^{*}-s\right)-a_{0} \cdot s\right)} e^{-\xi\left(B\left(t^{*}-s\right)+K q\left(t^{*}-s\right)-\lambda\right)} d s, \\
& J_{\xi}=\int_{\left|s-t^{*}\right| \geq \delta_{0}} e^{i \xi\left(p\left(t^{*}-s\right)-a_{0} . s\right)} e^{-\xi\left(B\left(t^{*}-s\right)+K q\left(t^{*}-s\right)-\lambda\right)} d s .
\end{aligned}
$$

Vamos fazer estimativas para as sequências $I_{\xi}$ e $J_{\xi}$ com a finalidade de mostrar que os coeficientes $u\left(t^{*}, \xi\right)$ crescem muito rápido quando $\xi$ tende ao infinito.

Lembrando as propriedades das funções $p$ e $q$, para $\left|s-t^{*}\right| \leq \delta$ temos $q\left(s-t^{*}\right)=$ $\left|s-t^{*}\right|^{2}$ e $p\left(s-t^{*}\right)=a_{0} \cdot s$. Portanto, como $\delta_{0}<\delta$, fazendo a mudança de variáveis $\sigma=\sqrt{K \xi}\left(t^{*}-s\right)$ obtemos 


$$
\begin{aligned}
I_{\xi} & =\int_{\left|s-t^{*}\right|<\delta_{0}} e^{-\xi\left(B\left(t^{*}-s\right)+K\left|t^{*}-s\right|^{2}-\lambda\right)} d s \\
& \geq e^{\xi \lambda / 2} \int_{\left|s-t^{*}\right|<\delta_{0}} e^{-\xi K\left|t^{*}-s\right|^{2}} d s \\
& =e^{\xi \lambda / 2} \frac{1}{(K \xi)^{n / 2}} \int_{|\sigma|<\delta_{0} \sqrt{K \xi}} e^{-|\sigma|^{2}} d \sigma \\
& =e^{\xi \lambda / 2} \frac{\mu_{\xi}}{(K \xi)^{n / 2}} \geq e^{\xi \lambda / 2} \frac{\mu_{1}}{(K \xi)^{n / 2}},
\end{aligned}
$$

sendo $\mu_{\xi}=\int_{|\sigma|<\delta_{0} \sqrt{K \xi}} e^{-|\sigma|^{2}} d \sigma, \xi \in \mathbb{Z}_{+} \backslash \mathcal{G}$, uma sequência crescente de números reais positivos.

Por outro lado, existe uma constante $C>0$ tal que $\left|J_{\xi}\right| \leq C e^{-\xi \lambda / 2}$ para todo $\xi \in \mathbb{Z}_{+} \backslash \mathcal{G}$. Com efeito, considere o cubo $Q^{*}=t^{*}-[0,2 \pi]^{n}$ e a constante $\sigma \doteq$ $\min \left\{q(\tau) ; \tau \in Q^{*} \mathrm{e}|\tau| \geq \delta_{0}\right\}$, dessa forma temos $\sigma>0$. Portanto,

$$
\begin{aligned}
\left|J_{\xi}\right| & \leq \int_{\left|s-t^{*}\right| \geq \delta_{0}}\left|e^{i \xi\left(p\left(t^{*}-s\right)-a_{0} \cdot s\right)}\right| e^{-\xi\left(B\left(t^{*}-s\right)+K q\left(t^{*}-s\right)-\lambda\right)} d s \\
& =\int_{\left|s-t^{*}\right| \geq \delta_{0}} e^{-\xi\left(B\left(t^{*}-s\right)+K q\left(t^{*}-s\right)-\lambda\right)} d s \\
& \leq \int_{\left|s-t^{*}\right| \geq \delta_{0}} e^{-\xi\left(B\left(t^{*}-s\right)+K \sigma-\lambda\right)} d s
\end{aligned}
$$

Escolhemos agora $K>\sigma^{-1}(3 \lambda / 2-\mu) \operatorname{com} \mu \doteq \min \left\{B\left(t^{*}-s\right) ;\left|s-t^{*}\right| \geq \delta_{0}\right\}$. Logo, da desigualdade anterior temos

$$
\left|J_{\xi}\right| \leq \int_{\left|s-t^{*}\right| \geq \delta_{0}} e^{-\xi \lambda / 2} d s=C e^{-\xi \lambda / 2}
$$

Escolhemos também $\xi_{0} \in \mathbb{N}$ tal que

$$
e^{\xi \lambda / 4} \geq \widetilde{C} \xi^{n / 2}, \quad \forall \xi \geq \xi_{0}
$$

sendo $\widetilde{C}=\frac{K^{n / 2}}{\mu_{1}}(1+C)$. Assim,

$$
e^{\xi \lambda / 4} \geq \widetilde{C} \xi^{n / 2} \geq \frac{K^{n / 2}}{\mu_{1}}\left(1+\frac{C}{e^{\xi 3 \lambda / 4}}\right) \xi^{n / 2} .
$$

Multiplicando a desigualdade acima por $e^{\xi \lambda / 4}$ obtemos

$$
\frac{\mu_{1}}{(K \xi)^{n / 2}} e^{\xi \lambda / 2}-C e^{-\xi \lambda / 2} \geq e^{\xi \lambda / 4}
$$


e segue que

$$
\left|\hat{u}\left(t^{*}, \xi\right)\right| \geq\left|I_{\xi}\right|-\left|J_{\xi}\right| \geq \frac{\mu_{1}}{(K \xi)^{n / 2}} e^{\xi \lambda / 2}-C e^{-\xi \lambda / 2} \geq e^{\xi \lambda / 4}, \quad \forall \xi \geq \xi_{0}, \quad \xi \in \mathbb{Z}_{+} \backslash \mathcal{G} .
$$

Portanto, a sequência $\hat{u}(\cdot, \xi)$ não é uma sequência de coeficientes parciais de Fourier de nenhuma distribuição $u \in \mathcal{D}^{\prime}\left(\mathbb{T}^{n+1}\right)$. Encerramos assim a demonstração no caso 1 .

$$
\text { Caso 2: }\left\{c_{10}, \ldots, c_{n 0}\right\} \cap \mathbb{Z} \neq \emptyset
$$

Ainda estamos trabalhando sob a hipótese $c_{0} \notin \mathbb{Z}^{n}$. Vamos considerar agora o caso em que existem períodos $c_{j 0} \in \mathbb{Z}$.

Reordenando os campos $L_{j}$, podemos assumir que $c_{j 0} \notin \mathbb{Z}, j=1, \ldots, p$, e $c_{j 0} \in \mathbb{Z}$, $j=p+1, \ldots, n$, para algum $1 \leq p \leq n-1$. Então, o caso 1 juntamente com o Lema 2.10, dado a seguir, conclui a demonstração no caso 2.

Lema 2.10. Sejam $1 \leq p \leq n$ e $\mathbb{L}$ o operador dado em (2.2). Se o operador determinado pelos campos $L_{1}, \ldots, L_{p}$ não é globalmente resolúvel em $\mathbb{T}^{p+1}$ e $c_{(p+1) 0}, \ldots, c_{n 0} \in \mathbb{Z}$ então $\mathbb{L}$ não é globalmente resolúvel em $\mathbb{T}^{n+1}$.

Demonstração. Denotamos as coordenadas em $\mathbb{T}^{n+1}$ por $\left(t^{\prime}, t^{\prime \prime}, x\right)$, sendo $t^{\prime}=\left(t_{1}, \ldots, t_{p}\right)$ e $t^{\prime \prime}=\left(t_{p+1}, \ldots, t_{n}\right)$. Similarmente, usamos a notação $c_{0}=\left(c_{0}^{\prime}, c_{0}^{\prime \prime}\right)$, na qual $c_{0}^{\prime}=$ $\left(c_{10}, \ldots, c_{p 0}\right)$ e $c_{0}^{\prime \prime}=\left(c_{(p+1) 0}, \ldots, c_{n 0}\right)$.

Considere o operador $\mathbb{L}^{\#}$ determinado pelos campos vetoriais

$$
L_{j}=\frac{\partial}{\partial t_{j}}+\left(a_{j 0}+i b_{j}\left(t_{j}\right)\right) \frac{\partial}{\partial x}, \quad j=1, \ldots, p,
$$

e $\mathbb{E}^{\#}$ seu correspondente espaço de condições de compatibilidade, conforme definido em (1.5). Uma vez que $\mathbb{L}^{\#}$ não é globalmente resolúvel em $\mathbb{T}^{p+1}$, existe $g\left(t^{\prime}, x\right)=$ $\sum_{j=1}^{p} g_{j}\left(t^{\prime}, x\right) d t_{j} \in \mathbb{E}^{\#}$ tal que a equação $\mathbb{L}^{\#} v=g$ não possui solução $v \in \mathcal{D}^{\prime}\left(\mathbb{T}^{p+1}\right)$.

Seja $B(t) \doteq \mathcal{B}_{1}\left(t^{\prime}\right)+\mathcal{B}_{2}\left(t^{\prime \prime}\right)$ uma primitiva global do pull-back $\Pi^{*} b$, definida no recobrimento minimal $\mathcal{T}=\mathbb{R}^{r} \times \mathbb{T}^{n-r}$, sendo $r$ o posto do grupo de períodos da 1 forma $b$. Como por hipótese $c_{0}^{\prime \prime} \in \mathbb{Z}^{n-p}$ (o que equivale a $b_{0}^{\prime \prime}=0$ e $a_{0}^{\prime \prime} \in \mathbb{Z}^{n-p}$ ) temos que $0 \leq r \leq p$ e que $\mathcal{B}_{2}$ está definida em $\mathbb{T}^{n-p}$.

Definimos agora as funções

$$
f_{j}(t, x) \doteq \sum_{\xi \in \mathbb{Z}} \hat{f}_{j}(t, \xi) e^{i \xi x}, \quad j=1, \ldots, p
$$

nas quais, os coeficientes $\hat{f}_{j}(\cdot, \xi)$ são dados por

$$
\hat{f}_{j}(t, \xi) \doteq\left\{\begin{array}{ll}
\hat{g}_{j}\left(t^{\prime}, \xi\right) e^{\xi\left(\mathcal{B}_{2}\left(t^{\prime \prime}\right)-M-i a_{0}^{\prime \prime} \cdot t^{\prime \prime}\right)} & \text { se } \xi \geq 0 \\
\hat{g}_{j}\left(t^{\prime}, \xi\right) e^{\xi\left(\mathcal{B}_{2}\left(t^{\prime \prime}\right)-m-i a_{0}^{\prime \prime} \cdot t^{\prime \prime}\right)} & \text { se } \xi<0
\end{array},\right.
$$


sendo $M \doteq \max \left\{\mathcal{B}_{2}\left(t^{\prime \prime}\right) ; t^{\prime \prime} \in \mathbb{T}^{n-p}\right\}$ e $m \doteq \min \left\{\mathcal{B}_{2}\left(t^{\prime \prime}\right) ; t^{\prime \prime} \in \mathbb{T}^{n-p}\right\}$.

Escolhemos também

$$
f_{j} \equiv 0, \quad j=p+1, \ldots, n \text {. }
$$

Dado $\alpha=\left(\alpha_{1}, \ldots, \alpha_{n}\right) \in \mathbb{Z}_{+}^{n}$, para cada $j=1, \ldots, p$ obtemos a igualdade

$$
\partial^{\alpha} \hat{f}_{j}(t, \xi)=\left(\partial^{\left(\alpha_{1}, \ldots, \alpha_{p}\right)} g_{j}\left(t^{\prime}, \xi\right)\right) P\left(t^{\prime \prime}, \xi\right) e^{\xi\left(\mathcal{B}_{2}\left(t^{\prime \prime}\right)-M-i a_{0}^{\prime \prime} \cdot t^{\prime \prime}\right)}, \quad \xi \in \mathbb{Z}_{+},
$$

na qual $P$ é um polinômio envolvendo apenas potências de $\xi$ (potências de grau no máximo $\left.\left|\alpha^{\prime \prime}\right| \doteq \alpha_{p+1}+\cdots+\alpha_{n}\right)$ e derivadas de $\mathcal{B}_{2}\left(t^{\prime \prime}\right)-i a_{0}^{\prime \prime} \cdot t^{\prime \prime}$ as quais são limitadas em $\mathbb{T}^{n-p}$. Deste modo, existe uma constante $C_{\alpha}>0$ tal que $\left|P\left(t^{\prime \prime}, \xi\right)\right| \leq C_{\alpha}|\xi|^{\left|\alpha^{\prime \prime}\right|}$ para todo $t^{\prime \prime}$. Portanto,

$$
\left|\partial^{\alpha} \hat{f}_{j}(t, \xi)\right| \leq C_{\alpha}|\xi|^{\left|\alpha^{\prime \prime}\right|}\left|\partial^{\left(\alpha_{1}, \ldots, \alpha_{p}\right)} g_{j}\left(t^{\prime}, \xi\right)\right|, \quad \xi \in \mathbb{Z}_{+}
$$

Analogamente, obtemos a mesma desigualdade para $\xi \in \mathbb{Z}_{-}$. Como $g_{j}$ são funções suaves é possível concluir, pela desigualdade anterior, que as funções $f_{j}$ também são suaves.

Finalmente definimos $f=\sum_{j=1}^{n} f_{j} d t_{j}$. Vejamos que $f \in \mathbb{E}$.

Primeiramente precisamos verificar que $L_{j} f_{k}=L_{k} f_{j}, j, k=1, \ldots, n$. Para $j, k=$ $1, \ldots, p$ facilmente vemos que $L_{j} f_{k}=L_{k} f_{j}$ uma vez que $L_{j} g_{k}=L_{k} g_{j}$. Para $j, k=$ $p+1, \ldots, n$ temos $L_{j} f_{k}=L_{k} f_{j}=0$. Resta-nos verificar que $L_{j} f_{k}=L_{k} f_{j}$ quando $j=1, \ldots, p$ e $k=p+1, \ldots, n$. Neste caso,

$$
\begin{gathered}
\widehat{L_{k} f_{j}}(t, \xi)=\left(b_{k}\left(t_{k}\right)-i a_{k 0}\right) \hat{g}_{j}\left(t^{\prime}, \xi\right) e^{\xi\left(\mathcal{B}\left(t^{\prime \prime}\right)-M-i a_{0}^{\prime \prime} \cdot t^{\prime \prime}\right)}+ \\
+i \xi\left(a_{k 0}+i b_{k}\left(t_{k}\right)\right) \hat{g}_{j}(t, \xi) e^{\xi\left(\mathcal{B}\left(t^{\prime \prime}\right)-M-i a_{0}^{\prime \prime} \cdot t^{\prime \prime}\right)}=0=\widehat{L_{j} f_{k}}(t, \xi), \quad \forall t \in \mathbb{T}^{n}, \quad \forall \xi \geq 1 .
\end{gathered}
$$

\left. Analogamente ${\widehat{L_{k} f_{j}}}_{j}(t, \xi)=0={\widehat{L_{j} f_{k}}}_{(t}, \xi\right), \forall t \in \mathbb{T}^{n}, \forall \xi \leq-1$. Quando $\xi=0$ é direto.

Vejamos agora que $f$ cumpre a outra condição de compatibilidade. Com efeito, a função suave e periódica $C(t)=i\left(B(t)-b_{0} \cdot t\right)$ satisfaz

$$
d_{t} C=i\left(b-b_{0}\right)=a_{0}+i b-\left(a_{0}+i b_{0}\right)=c-c_{0}
$$

sendo $c(t)=\sum_{j=1}^{n}\left(a_{j 0}+i b_{j}\left(t_{j}\right)\right) d t_{j}$ e $c_{0}=\sum_{j=1}^{n}\left(a_{j 0}+i b_{j 0}\right) d t_{j}$. Afirmamos que a 1-forma $\hat{f}(t, \xi) e^{i \xi\left(c_{0} \cdot t+C(t)\right)}$ é exata sempre que $\xi \in \mathbb{Z}$ satisfaz $\xi c_{0} \in \mathbb{Z}^{n}$.

De fato, se $\xi \in \mathbb{Z}$ é tal que $\xi\left(c_{0}^{\prime}, c_{0}^{\prime \prime}\right) \in \mathbb{Z}^{n}$ então $\xi c_{0}^{\prime} \in \mathbb{Z}^{p}$, logo $\hat{g}\left(t^{\prime}, \xi\right) e^{i \xi\left(c_{0}^{\prime} \cdot t^{\prime}+C_{1}\left(t^{\prime}\right)\right)}$ é exata, sendo $C_{1}\left(t^{\prime}\right)=i\left(\mathcal{B}_{1}\left(t^{\prime}\right)-b_{0}^{\prime} \cdot t^{\prime}\right)$. Ou seja, existe uma função $\gamma_{\xi} \in C^{\infty}\left(\mathbb{T}_{t^{\prime}}^{p}\right)$ tal 
que

$$
\begin{aligned}
d_{t^{\prime}} \gamma_{\xi}\left(t^{\prime}\right) & =\hat{g}\left(t^{\prime}, \xi\right) e^{i \xi\left(c_{0}^{\prime} \cdot t^{\prime}+C_{1}\left(t^{\prime}\right)\right)} \\
& =\hat{g}\left(t^{\prime}, \xi\right) e^{i \xi\left(a_{0}^{\prime} \cdot t^{\prime}+i \mathcal{B}_{1}\left(t^{\prime}\right)\right)} \\
& =\hat{g}\left(t^{\prime}, \xi\right) e^{i \xi\left(-a_{0}^{\prime \prime} \cdot t^{\prime \prime}-i \mathcal{B}_{2}\left(t^{\prime \prime}\right)\right)} e^{i \xi\left(a_{0} \cdot t+i B(t)\right)} \\
& =\hat{g}\left(t^{\prime}, \xi\right) e^{i \xi\left(-a_{0}^{\prime \prime} \cdot t^{\prime \prime}-i \mathcal{B}_{2}\left(t^{\prime \prime}\right)\right)} e^{i \xi\left(c_{0} \cdot t+C(t)\right)}
\end{aligned}
$$

Se $\xi \geq 0$ multiplicamos a equação acima por $e^{-\xi M}$ e obtemos:

$$
d_{t^{\prime}}\left(e^{-\xi M} \gamma_{\xi}\left(t^{\prime}\right)\right)=\hat{f}(t, \xi) e^{i \xi\left(c_{0} \cdot t+C(t)\right)} .
$$

Se $\xi<0$ multiplicamos por $e^{-\xi m}$, e portanto

$$
d_{t^{\prime}}\left(e^{-\xi m} \gamma_{\xi}\left(t^{\prime}\right)\right)=\hat{f}(t, \xi) e^{i \xi\left(c_{0} \cdot t+C(t)\right)} \text {. }
$$

Dessa forma, concluímos que $f \in \mathbb{E}$.

Suponha por absurdo que existe $u \in \mathcal{D}^{\prime}\left(\mathbb{T}^{n+1}\right)$ tal que $\mathbb{L} u=f$. Se $u(t, x)=$ $\sum_{\xi \in \mathbb{Z}} \hat{u}(t, \xi) e^{i \xi x}$, para cada $\xi \in \mathbb{Z}$, obtemos as equações

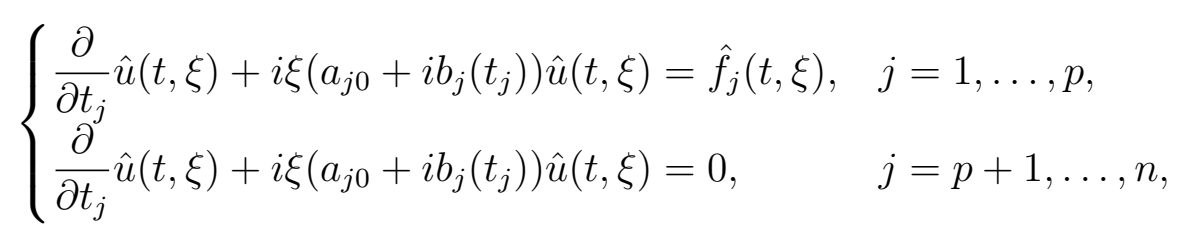

as quais podem ser reescritas na seguinte forma

$$
\begin{cases}\frac{\partial}{\partial t_{j}} w(t, \xi)+i \xi\left(a_{j 0}+i b_{j}\left(t_{j}\right)\right) w(t, \xi)=e^{i \xi a_{0}^{\prime \prime} \cdot t^{\prime \prime}} \hat{f}_{j}(t, \xi), & j=1, \ldots, p \\ \frac{\partial}{\partial t_{j}} w(t, \xi)-\xi b_{j}\left(t_{j}\right) w(t, \xi)=0, & j=p+1, \ldots, n\end{cases}
$$

sendo $w(t, \xi) \doteq \hat{u}(t, \xi) e^{i \xi a_{0}^{\prime \prime} \cdot t^{\prime \prime}}$. De acordo com cada $\xi \in \mathbb{Z}$, para $j=p+1, \ldots, n$ temos

$$
\begin{aligned}
\frac{\partial}{\partial t_{j}}\left(w(t, \xi) e^{-\xi\left(\mathcal{B}_{2}\left(t^{\prime \prime}\right)-M\right)}\right) & =0, \quad \text { se } \xi \geq 0, \\
\frac{\partial}{\partial t_{j}}\left(w(t, \xi) e^{-\xi\left(\mathcal{B}_{2}\left(t^{\prime \prime}\right)-m\right)}\right) & =0, \quad \text { se } \xi<0 .
\end{aligned}
$$

Assim, existem funções $\phi_{\xi}, \xi \in \mathbb{Z}$, tais que

$$
\begin{aligned}
& w(t, \xi) e^{-\xi\left(\mathcal{B}_{2}\left(t^{\prime \prime}\right)-M\right)} \doteq \phi_{\xi}\left(t^{\prime}\right), \quad \xi \geq 0, \\
& w(t, \xi) e^{-\xi\left(\mathcal{B}_{2}\left(t^{\prime \prime}\right)-m\right)} \doteq \phi_{\xi}\left(t^{\prime}\right), \quad \xi<0
\end{aligned}
$$


Vejamos que

$$
\varphi\left(t^{\prime}, x\right)=\sum_{\xi \in \mathbb{Z}} \phi_{\xi}\left(t^{\prime}\right) e^{i \xi x}
$$

define uma distribuição em $\mathbb{T}^{p+1}$. De fato, sejam $t_{M}^{\prime \prime}$ e $t_{m}^{\prime \prime} \in \mathbb{T}^{n-p}$ tais que $\mathcal{B}_{2}\left(t_{M}^{\prime \prime}\right)=M$ e $\mathcal{B}_{2}\left(t_{m}^{\prime \prime}\right)=m$. Então, dado $\beta \in \mathbb{Z}_{+}^{p}$ segue que

$$
\begin{aligned}
& \left|\partial^{\beta} \phi_{\xi}\left(t^{\prime}\right)\right|=\left|\partial^{\beta} w\left(t^{\prime}, t_{M}^{\prime \prime}, \xi\right)\right|=\left|\partial^{\beta} \hat{u}\left(t^{\prime}, t_{M}^{\prime \prime}, \xi\right)\right|, \quad \forall \xi \geq 0, \\
& \left|\partial^{\beta} \phi_{\xi}\left(t^{\prime}\right)\right|=\left|\partial^{\beta} w\left(t^{\prime}, t_{m}^{\prime \prime}, \xi\right)\right|=\left|\partial^{\beta} \hat{u}\left(t^{\prime}, t_{m}^{\prime \prime}, \xi\right)\right|, \quad \forall \xi<0 .
\end{aligned}
$$

Portanto $\varphi \in \mathcal{D}^{\prime}\left(\mathbb{T}^{p+1}\right)$, uma vez que $u$ é distribuição.

Por outro lado, para $j=1, \ldots, p$ vimos que

$$
\frac{\partial}{\partial t_{j}} w(t, \xi)+i \xi\left(a_{j 0}+i b_{j}\left(t_{j}\right)\right) w(t, \xi)=e^{i \xi a_{0}^{\prime \prime} \cdot t^{\prime \prime}} \hat{f}_{j}(t, \xi), \quad \xi \in \mathbb{Z} .
$$

Substituindo (2.20) na equação anterior, obtemos

$$
\begin{gathered}
\frac{\partial}{\partial t_{j}} \phi_{\xi}\left(t^{\prime}\right)+i \xi\left(a_{j 0}+i b_{j}\left(t_{j}\right)\right) \phi_{\xi}\left(t^{\prime}\right)=e^{-\xi\left(\mathcal{B}_{2}\left(t^{\prime \prime}\right)-M\right)} e^{i \xi a_{0}^{\prime \prime} \cdot t^{\prime \prime}} \hat{f}_{j}(t, \xi)=\hat{g}_{j}\left(t^{\prime}, \xi\right), \quad \xi \geq 0 \\
\frac{\partial}{\partial t_{j}} \phi_{\xi}\left(t^{\prime}\right)+i \xi\left(a_{j 0}+i b_{j}\left(t_{j}\right)\right) \phi_{\xi}\left(t^{\prime}\right)=e^{-\xi\left(\mathcal{B}_{2}\left(t^{\prime \prime}\right)-m\right)} e^{i \xi a_{0}^{\prime \prime} \cdot t^{\prime \prime}} \hat{f}_{j}(t, \xi)=\hat{g}_{j}\left(t^{\prime}, \xi\right), \quad \xi<0
\end{gathered}
$$

donde concluímos que $L_{j} \varphi=g_{j}$ para $j=1, \ldots, p$, ou seja, $\mathbb{L}^{\#} \varphi=g$, o que é um absurdo.

\subsection{Proposição 2.4: demonstração da suficiência}

Na Proposição 2.3 tratamos da resolubilidade global do operador $\mathbb{L}$, definido em (2.2), quando todas as funções $b_{j}$ são não identicamente nulas. Naquele caso, vimos que a parte real $a_{0}$ interfere na resolubilidade global de $\mathbb{L}$ somente quando a 1-forma $b$ é exata. Já na Proposição 2.4, onde consideramos a existência de funções $b_{j}$ identicamente nulas, veremos que a parte real $a_{0}$ pode ser determinante na resolubilidade global de $\mathbb{L}$ independente de $b$ ser exata ou não.

Semelhantemente à Proposição 2.3, a existência de uma função $b_{j} \not \equiv 0$ que não muda de sinal, é suficiente para que $\mathbb{L}$ seja globalmente resolúvel. A demonstração é análoga à feita na Seção 2.1.1. Assim, nos resta provar que cada uma das condições (ii) e (iii) é suficiente para $\mathbb{L}$ ser globalmente resolúvel.

Para cada $k=1, \ldots, m$ considere o difeomorfismo

$$
\left(t_{1}, \ldots, t_{k}, \ldots, t_{j_{k}}, \ldots, t_{n}, x\right) \mapsto\left(t_{1}, \ldots, t_{j_{k}}, \ldots, t_{k}, \ldots, t_{n}, x\right)
$$


Estes difeomorfismos produzem novas coordenadas em $\mathbb{T}^{n+1}$, nas quais

$$
J \doteq\left\{j=1, \ldots, n ; b_{j} \equiv 0\right\}=\{1, \ldots, m\}
$$

Além disso, a estrutura localmente integrável gerada pelos novos campos permanece a mesma anterior uma vez que só houve uma reordenação dos campos $L_{j}$.

\subsubsection{Condição suficiente $(i i)$}

Suponhamos que $\left(a_{10}, \ldots, a_{m 0}\right) \notin \mathbb{Q}^{m}$ é não-Liouville. Assim, existe uma constante $C>0$ e um número inteiro $s>1$ tal que

$$
\max _{1 \leq j \leq m}\left|\xi a_{j 0}-\kappa_{j}\right| \geq \frac{C}{|\xi|^{s-1}}, \quad \forall(\kappa, \xi) \in \mathbb{Z}^{m} \times \mathbb{N} .
$$

Seja $f=\sum_{j=1}^{n} f_{j} d t_{j} \in \mathbb{E}$. No decorrer desta seção, denotaremos por $t=\left(t^{\prime}, t^{\prime \prime}, x\right)$ as coordenadas em $\mathbb{T}^{n+1}$, sendo $t^{\prime}=\left(t_{1}, \ldots, t_{m}\right)$ e $t^{\prime \prime}=\left(t_{m+1}, \ldots, t_{n}\right)$.

Usaremos série parcial de Fourier nas variáveis $\left(t^{\prime}, x\right)$ para obter uma solução $u$ da equação $\mathbb{L} u=f$. Considere então as séries formais

$$
u(t, x)=\sum_{(\kappa, \xi) \in \mathbb{Z}^{m} \times \mathbb{Z}} \hat{u}\left(t^{\prime \prime}, \kappa, \xi\right) e^{i\left(\kappa \cdot t^{\prime}+\xi x\right)}
$$

$\mathrm{e}$

$$
f_{j}(t, x)=\sum_{(\kappa, \xi) \in \mathbb{Z}^{m} \times \mathbb{Z}} \hat{f}_{j}\left(t^{\prime \prime}, \kappa, \xi\right) e^{i\left(\kappa \cdot t^{\prime}+\xi x\right)} j=1, \ldots, n .
$$

Substituindo (2.22) e (2.23) nas equações $L_{j} u=f_{j}, j=1, \ldots, m$, obtemos para cada $(\kappa, \xi) \neq(0,0)$ as equações

$$
i\left(\kappa_{j}+\xi a_{j 0}\right) \hat{u}\left(t^{\prime \prime}, \kappa, \xi\right)=\hat{f}_{j}\left(t^{\prime \prime}, \kappa, \xi\right), \quad j=1, \ldots, m
$$

Além disso, da condição de compatibilidade $\mathbb{L} f=0$ temos $L_{j} f_{\ell}=L_{\ell} f_{j}, j, \ell=$ $1, \ldots m$, donde obtemos as equações

$$
\left(\kappa_{j}+\xi a_{j 0}\right) \hat{f}_{\ell}\left(t^{\prime \prime}, \kappa, \xi\right)=\left(\kappa_{\ell}+\xi a_{\ell 0}\right) \hat{f}_{j}\left(t^{\prime \prime}, \kappa, \xi\right), \quad j, \ell=1, \ldots, m
$$

Portanto, as equações anteriores implicam a seguinte igualdade

$$
\hat{u}\left(t^{\prime \prime}, \kappa, \xi\right)=\frac{1}{i\left(\kappa_{M}+\xi a_{M 0}\right)} \hat{f}_{M}\left(t^{\prime \prime}, \kappa, \xi\right), \quad(\kappa, \xi) \neq(0,0),
$$

sendo $1 \leq M \leq m, M=M(\xi)$, tal que

$$
\left|\kappa_{M}+\xi a_{M 0}\right| \doteq \max _{1 \leq j \leq m}\left|\kappa_{j}+\xi a_{j 0}\right| .
$$


Uma vez que $f \in \mathbb{E}$ segue que $\hat{f}\left(t^{\prime \prime}, 0,0\right)$ é exata (ver definição de $\mathbb{E}$ em (1.5)). Desse modo, existe uma função suave $v$ definida em $\mathbb{T}_{t^{\prime \prime}}^{n-m}$ tal que $d_{t^{\prime \prime}} v=\hat{f}(\cdot, 0,0)$. Escolhemos então $\hat{u}\left(t^{\prime \prime}, 0,0\right)=v\left(t^{\prime \prime}\right)$.

Vejamos que a série $u$ definida por (2.22), com coeficientes $\hat{u}(\cdot, \kappa, \xi)$ dados por $(2.24)$ e $\hat{u}\left(t^{\prime \prime}, 0,0\right)=v\left(t^{\prime \prime}\right)$, é uma solução suave da equação $\mathbb{L} u=f$.

Dado $\alpha \in \mathbb{Z}_{+}^{n-m}$, a desigualdade (2.21) juntamente com a fórmula (2.24) resultam em

$$
\left|\partial^{\alpha} \hat{u}\left(t^{\prime \prime}, \kappa, \xi\right)\right| \leq \frac{1}{C}|\xi|^{s-1}\left|\partial^{\alpha} \hat{f}_{M}\left(t^{\prime \prime}, \kappa, \xi\right)\right|, \quad(\kappa, \xi) \neq(0,0)
$$

Uma vez que cada função $f_{j}$ é suave, dado um inteiro positivo $L$, existe uma constante $C_{\alpha}>0$ tal que pela desigualdade acima obtemos

$$
\begin{aligned}
\left|\partial^{\alpha} \hat{u}\left(t^{\prime \prime}, \kappa, \xi\right)\right| & \leq \frac{1}{C}|\xi|^{s-1}\left|\partial^{\alpha} \hat{f}_{M}\left(t^{\prime \prime}, \kappa, \xi\right)\right| \\
& \leq C_{\alpha} \frac{1}{(1+|(\kappa, \xi)|)^{L}}, \quad(\kappa, \xi) \neq(0,0)
\end{aligned}
$$

donde concluímos que $u \in C^{\infty}\left(\mathbb{T}^{n+1}\right)$. Da forma que foi construída, a função $u$ satisfaz $L_{j} u=f_{j}, j=1, \ldots, m$. Verifiquemos que $u$ também satisfaz as equações

$$
L_{j} u=f_{j}, \quad j=m+1, \ldots, n .
$$

Seja $j=m+1, \ldots, n$. Dado $(\kappa, \xi) \neq(0,0)$, podemos escrever

$$
\hat{u}\left(t^{\prime \prime}, \kappa, \xi\right)=\frac{1}{i\left(\kappa_{M}+\xi a_{M 0}\right)} \hat{f}_{M}\left(t^{\prime \prime}, \kappa, \xi\right),
$$

conforme (2.24). Além disso, da condição de compatibilidade $L_{j} f_{M}=L_{M} f_{j}$ temos

$$
i\left(\kappa_{M}+\xi a_{M 0}\right) \hat{f}_{j}\left(t^{\prime \prime}, \kappa, \xi\right)=\frac{\partial}{\partial t_{j}} \hat{f}_{M}\left(t^{\prime \prime}, \kappa, \xi\right)+i \xi c_{j}\left(t_{j}\right) \hat{f}_{M}\left(t^{\prime \prime}, \kappa, \xi\right) .
$$

Assim, as duas últimas igualdades implicam

$$
\begin{aligned}
& \frac{\partial}{\partial t_{j}} \hat{u}\left(t^{\prime \prime}, \kappa, \xi\right)+i \xi c_{j}\left(t_{j}\right) \hat{u}\left(t^{\prime \prime}, \kappa, \xi\right)= \\
= & \frac{1}{i\left(\kappa_{M}+\xi a_{M 0}\right)} \frac{\partial}{\partial t_{j}} \hat{f}_{M}\left(t^{\prime \prime}, \kappa, \xi\right)+i \xi c_{j}\left(t_{j}\right) \frac{1}{i\left(\kappa_{M}+\xi a_{M 0}\right)} \hat{f}_{M}\left(t^{\prime \prime}, \kappa, \xi\right) \\
= & \frac{1}{i\left(\kappa_{M}+\xi a_{M 0}\right)}\left(\frac{\partial}{\partial t_{j}} \hat{f}_{M}\left(t^{\prime \prime}, \kappa, \xi\right)+i \xi c_{j}\left(t_{j}\right) \hat{f}_{M}\left(t^{\prime \prime}, \kappa, \xi\right)\right) \\
= & \hat{f}_{j}\left(t^{\prime \prime}, \kappa, \xi\right) .
\end{aligned}
$$

Finalmente, para $(\kappa, \xi)=(0,0)$ temos $\frac{\partial}{\partial t_{j}} \hat{u}\left(t^{\prime \prime}, 0,0\right)=\hat{f}_{j}\left(t^{\prime \prime}, 0,0\right)$. 


\subsubsection{Condição suficiente $(i i i)$}

Vamos relembrar as hipóteses da condição $(i i i): b$ é exata, $a_{0} \in \mathbb{Q}^{n}, q_{J}=q_{*}$, e se $B$ é uma primitiva de $b$ então todos os subníveis e superníveis de $B$ são conexos em $\mathbb{T}^{n}$.

Seja $\mathcal{A} \doteq q_{*} \mathbb{Z}$ e $\mathcal{B} \doteq \mathbb{Z} \backslash \mathcal{A}$. Definimos o espaço

$$
\begin{aligned}
\mathcal{D}_{\mathcal{A}}^{\prime}\left(\mathbb{T}^{n+1}\right) & \doteq\left\{u \in \mathcal{D}^{\prime}\left(\mathbb{T}^{n+1}\right) ; \quad u(t, x)=\sum_{\xi \in \mathcal{A}} \hat{u}(t, \xi) e^{i \xi x}\right\} \\
& =\left\{u \in \mathcal{D}^{\prime}\left(\mathbb{T}^{n+1}\right) ; \quad u(t, x)=\sum_{N \in \mathbb{Z}} \hat{u}\left(t, q_{*} N\right) e^{i q_{*} N x}\right\} .
\end{aligned}
$$

Analogamente definimos $\mathcal{D}_{\mathcal{B}}^{\prime}\left(\mathbb{T}^{n+1}\right)$.

Denotamos por $\mathbb{L}_{\mathcal{A}}$ o operador $\mathbb{L}$ agindo sobre $\mathcal{D}_{\mathcal{A}}^{\prime}\left(\mathbb{T}^{n+1}\right)$. Similarmente, usamos a notação $\mathbb{L}_{\mathcal{B}}$ para o operador $\mathbb{L}$ agindo sobre $\mathcal{D}_{\mathcal{B}}^{\prime}\left(\mathbb{T}^{n+1}\right)$.

Pelo trabalho [BCP1] temos o seguinte resultado:

Lema 2.11. O operador $\mathbb{L}$ é globalmente resolúvel se, e somente se, os operadores $\mathbb{L}_{\mathcal{A}}$ $e \mathbb{L}_{\mathcal{B}}$ são globalmente resolúveis.

Lema 2.12. O operador $\mathbb{L}_{\mathcal{A}}$ é globalmente resolúvel.

Demonstração. Como $q_{*} a_{0} \in \mathbb{Z}^{n}$, podemos definir a seguinte aplicação

$$
\begin{aligned}
T: \mathcal{D}_{\mathcal{A}}^{\prime}\left(\mathbb{T}^{n+1}\right) & \longrightarrow \mathcal{D}_{\mathcal{A}}^{\prime}\left(\mathbb{T}^{n+1}\right) \\
\sum_{\xi \in \mathbb{Z}} \hat{u}\left(t, q_{*} \xi\right) e^{i q_{*} \xi x} & \longmapsto \sum_{\xi \in \mathbb{Z}} \hat{u}\left(t, q_{*} \xi\right) e^{-i q_{*} \xi a_{0} \cdot t} e^{i q_{*} \xi x}
\end{aligned}
$$

A aplicação $T$ define um automorfismo de $\mathcal{D}_{\mathcal{A}}^{\prime}\left(\mathbb{T}^{n+1}\right)$ e $C_{\mathcal{A}}^{\infty}\left(\mathbb{T}^{n+1}\right)$. Além disso, vale a seguinte conjugação

$$
T^{-1} \mathbb{L}_{\mathcal{A}} T=\mathbb{L}_{0, \mathcal{A}}
$$

sendo $\mathbb{L}_{0} \doteq d_{t}+i b(t) \wedge \frac{\partial}{\partial x}$.

Seja $B$ uma primitiva global da 1-forma $b$. Por hipótese, $B$ possui apenas subníveis e superníveis conexos em $\mathbb{T}^{n}$, logo o operador $\mathbb{L}_{0}$ é globalmente resolúvel pelo trabalho $[\mathrm{CH}]$. Portanto, $\mathbb{L}_{0, \mathcal{A}}$ é globalmente resolúvel e pela conjugação $T^{-1} \mathbb{L}_{\mathcal{A}} T=\mathbb{L}_{0, \mathcal{A}}$ concluímos que $\mathbb{L}_{\mathcal{A}}$ também é globalmente resolúvel.

Lema 2.13. O operador $\mathbb{L}_{\mathcal{B}}$ é globalmente resolúvel.

Demonstração. Seja $(\kappa, \xi) \in \mathbb{Z}^{m} \times \mathcal{B}$. Uma vez que $q_{J}=q_{*}$, ou seja, $q_{*}$ é o menor inteiro positivo que satisfaz $q_{*}\left(a_{10}, \ldots, a_{m 0}\right) \in \mathbb{Z}^{m}$, existe $j \in\{1, \ldots, m\}$ tal que 


$$
\left|a_{j 0}-\frac{\kappa_{j}}{\xi}\right| \geq \frac{C}{|\xi|}
$$

sendo $C=1 / q_{*}$. Assim,

$$
\max _{\ell=1, \ldots, m}\left|a_{\ell 0}-\frac{\kappa_{\ell}}{\xi}\right| \geq\left|a_{j 0}-\frac{\kappa_{j}}{\xi}\right| \geq \frac{C}{|\xi|}, \quad(\kappa, \xi) \in \mathbb{Z}^{m} \times \mathcal{B} .
$$

Ou seja, se os denominadores $\xi \in \mathcal{B}$ então $a_{0}^{\prime}=\left(a_{10}, \ldots, a_{m 0}\right)$ se comporta como não Liouville. Logo, semelhantemente à demonstração feita na Seção 2.3.1, concluímos que o operador $\mathbb{L}_{\mathcal{B}}$ é globalmente resolúvel.

\subsection{Proposição 2.4: demonstração da necessidade}

Nesta seção, mostraremos que quando $\emptyset \neq J \doteq\left\{j, b_{j} \equiv 0\right\} \neq\{1, \ldots, n\}$, o operador $\mathbb{L}$ definido por (2.2) será globalmente resolúvel se alguma das três condições apresentadas na Proposição 2.4 estiver satisfeita. Para isso, suponhamos que as condições $(i)$, (ii) e (iii) não são válidas, isto é, no decorrer desta seção todas as funções $b_{j} \not \equiv 0$ mudam de sinal e além disso temos dois casos a considerar. O primeiro será quando $\left(a_{j_{1} 0}, \ldots, a_{j_{m} 0}\right) \notin \mathbb{Q}^{m}$, neste caso vamos supor que $\left(a_{j_{1} 0}, \ldots, a_{j_{m} 0}\right)$ é Liouville. O outro será quando $\left(a_{j_{1} 0}, \ldots, a_{j_{m} 0}\right) \in \mathbb{Q}^{m}$, neste caso vamos supor que a condição $(i i i)$ não está satisfeita.

Como na seção anterior vamos assumir que $J=\{1, \ldots, m\}, 1 \leq m \leq n-1$. Além disso, continuamos denotando por $\left(t^{\prime}, t^{\prime \prime}, x\right)$ as coordenadas em $\mathbb{T}^{n+1}$, sendo $t^{\prime}=$ $\left(t_{1}, \ldots, t_{m}\right)$ e $t^{\prime \prime}=\left(t_{m+1}, \ldots, t_{n}\right)$. Usamos também a notação $c_{0}=\left(c_{0}^{\prime}, c_{0}^{\prime \prime}\right) \operatorname{com} c_{0}^{\prime}=$ $\left(c_{10}, \ldots, c_{m 0}\right)$ e $c_{0}^{\prime \prime}=\left(c_{(m+1) 0}, \ldots, c_{n 0}\right)$. A demonstração será feita de acordo com os casos a seguir:

Caso 1: $a_{0}^{\prime}=\left(a_{10}, \ldots, a_{m 0}\right) \notin \mathbb{Q}^{m}$ é Liouville;

Caso 2: $a_{0}^{\prime}=\left(a_{10}, \ldots, a_{m 0}\right) \in \mathbb{Q}^{m}$.

\subsubsection{Caso 1: $a_{0}^{\prime} \notin \mathbb{Q}^{m}$ é Liouville.}

Dentro do caso 1, consideramos dois subcasos, a saber:

- Caso 1.1: $c_{0}^{\prime \prime}=\left(c_{(m+1) 0}, \ldots, c_{n 0}\right) \in \mathbb{Q}^{n-m}$;

- Caso 1.2: $c_{0}^{\prime \prime}=\left(c_{(m+1) 0}, \ldots, c_{n 0}\right) \notin \mathbb{Q}^{n-m}$. 


\section{Caso 1.1: $c_{0}^{\prime \prime} \in \mathbb{Q}^{n-m}$}

Considere o operador $\mathbb{L}^{\#}$ associado aos campos vetoriais $L_{1}, \ldots, L_{m}$ e o conjunto $\mathcal{A}=\eta \mathbb{Z}$ no qual $\eta \in \mathbb{N}$ satisfaz $\eta c_{0}^{\prime \prime} \in \mathbb{Z}^{n-m}$. Uma vez que $a_{0}^{\prime} \notin \mathbb{Q}^{m}$ é Liouville, afirmamos que $\mathbb{L}_{\mathcal{A}}^{\#}$ não é globalmente resolúvel em $\mathbb{T}^{m+1}$. As ideias da demonstração dessa afirmação, que será apresentada a seguir, foram inspiradas no trabalho [BP].

Como $a_{0}^{\prime}$ é Liouville, pelos Lemas 1.6 e 1.7 é possível obter uma constante $C>0$ e uma sequência $\left\{\left(\kappa_{l}, \xi_{l}\right)\right\}$ em $(\eta \mathbb{Z})^{m} \times \eta \mathbb{Z}, \xi_{l} \geq 2$, tal que

$$
\max _{j=1, \ldots, m}\left|\xi_{l} a_{j 0}+\kappa_{l}^{(j)}\right|<C\left|\left(\kappa_{l}, \xi_{l}\right)\right|^{-l}, \quad \forall l \in \mathbb{N} .
$$

Seja $\gamma_{l} \doteq\left|\left(\kappa_{l}, \xi_{l}\right)\right|^{l / 2}, l \in \mathbb{N}$. Definimos

$$
v_{l}\left(t^{\prime}, x\right)=\gamma_{l} e^{i\left(\kappa_{l} \cdot t^{\prime}+\xi_{l} x\right)} \in C^{\infty}\left(\mathbb{T}^{m+1}\right), \quad l \in \mathbb{N} .
$$

Então para cada $j=1, \ldots, m$, obtemos

$$
L_{j} v_{l}=i \gamma_{l}\left(\kappa_{j}^{(j)}+\xi_{l} a_{j 0}\right) e^{i\left(\kappa_{l} \cdot t^{\prime}+\xi_{l} x\right)} \quad l \in \mathbb{N} .
$$

Definimos então

$$
g\left(t^{\prime}, x\right)=\sum_{l \in \mathbb{N}} \hat{g}\left(\kappa_{l}, \xi_{l}\right) e^{i\left(\kappa_{l} \cdot t^{\prime}+\xi_{l} x\right)},
$$

sendo

$$
\hat{g}\left(\kappa_{l}, \xi_{l}\right) \doteq i \gamma_{l} \sum_{j=1}^{m}\left(\kappa_{l}^{(j)}+\xi_{l} a_{j 0}\right) d t_{j} .
$$

Note que $g \in \wedge^{1} C_{\mathcal{A}}^{\infty}\left(\mathbb{T}^{m+1}\right)$ e pela construção garantimos que $g \in \mathbb{E}_{\mathcal{A}}^{\#}$. Suponha por absurdo que existe $u \in \mathcal{D}_{\mathcal{A}}^{\prime}\left(\mathbb{T}^{n+1}\right)$ tal que $\mathbb{L}_{\mathcal{A}}^{\#} u=g$. Portanto, como

$$
\mathbb{L}_{\mathcal{A}}^{\#} u=d_{t^{\prime}} u+a_{0}^{\prime} \wedge \frac{\partial}{\partial x} u
$$

se

segue que

$$
u(t, x)=\sum_{(\kappa, \xi) \in \mathcal{A}^{m} \times \mathcal{A}} \hat{u}(\kappa, \xi) e^{i\left(\kappa \cdot t^{\prime}+\xi x\right)}
$$

$$
\widehat{\mathbb{L}^{\#} u}\left(\kappa_{l}, \xi_{l}\right)=i \hat{u}\left(\kappa_{l}, \xi_{l}\right) \sum_{j=1}^{m}\left(\kappa_{l}^{(j)}+\xi_{l} a_{j 0}\right) d t_{j}=\hat{g}\left(\kappa_{l}, \xi_{l}\right), \quad \forall l \in \mathbb{N},
$$

donde concluímos que

$$
\widehat{u}\left(\kappa_{l}, \xi_{l}\right)=\gamma_{l}, \quad \forall l \in \mathbb{N},
$$

o que é uma absurdo pois a sequência $\left(\gamma_{l}\right)_{l \in \mathbb{N}}$ não corresponde a nenhuma distribuição definida em $\mathbb{T}^{m+1}$. Concluímos assim a demonstração da não resolubilidade de $\mathbb{L}_{\mathcal{A}}^{\#}$ em $\mathbb{T}^{m+1}$. Esse resultado juntamente com Lema 2.14, enunciado a seguir, concluem a demonstração no caso 1.1. 
Lema 2.14. Sejam $1 \leq p \leq n$ e $\mathbb{L}^{\#}$ o operador associado aos campos $L_{1}, \ldots, L_{p}$. Considere o conjunto $\mathcal{A}=\eta \mathbb{Z}$ no qual $\eta \in \mathbb{N}$. Sob estas condições, se $\mathbb{L}_{\mathcal{A}}^{\#}$ não é globalmente resolúvel em $\mathbb{T}^{p+1}$ e $\eta\left(c_{(p+1) 0}, \ldots, c_{n 0}\right) \in \mathbb{Z}^{n-p}$ então $\mathbb{L}_{\mathcal{A}}$ não é globalmente resolúvel em $\mathbb{T}^{n+1}$.

A demonstração do Lema 2.14 será omitida uma vez que é uma adaptação do Lema 2.10 .

\section{Caso 1.2: $c_{0}^{\prime \prime} \notin \mathbb{Q}^{n-m}$}

Assuma primeiramente que $\left\{c_{(m+1) 0}, \ldots, c_{n 0}\right\} \cap \mathbb{Q}=\emptyset$. Neste caso, a não resolubilidade de $\mathbb{L}$ será dada pelas Proposições 2.16 - 2.18 .

Seja $B$ uma primitiva global do pull-back $\Pi^{*} b$ através do recobrimento universal $\Pi: \mathbb{R}^{n} \rightarrow \mathbb{T}^{n}$. Uma vez que $b_{j} \equiv 0, j=1, \ldots, m$, a função $B$ pode ser escrita na seguinte forma $B(t)=B\left(t^{\prime \prime}\right)=\sum_{j=m+1}^{n} B_{j}\left(t_{j}\right)$ e como cada função $b_{j} \not \equiv 0$ muda de sinal, vamos assumir que o máximo da função $B=B\left(t^{\prime \prime}\right)$ não ocorre na fronteira do cubo $[0,2 \pi]_{t^{\prime \prime}}^{n-m}$ conforme feito no caso 1 da Seção 2.2. Assim, daqui em diante estaremos trabalhando nas seguintes condições:

$\star b_{j 0} \leq 0, j=m+1, \ldots, n$.

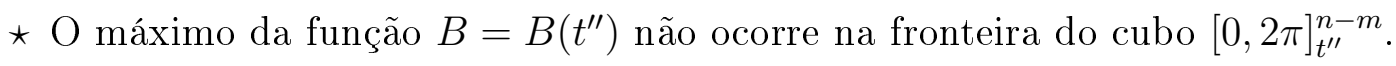

$\star B(0)=0$.

Ainda, relembrando as argumentações da Seção 2.2, vamos utilizar a condição

$$
h \doteq L_{\sigma(n)} \ldots L_{\sigma(2)} L_{\sigma(1)} u, \quad \sigma \in \mathcal{S}_{n}
$$

para construir uma 1-forma $f \in \mathbb{E}$ de modo que não exista solução $u \in \mathcal{D}^{\prime}\left(\mathbb{T}^{n+1}\right)$ da equação $\mathbb{L} u=f$. No entanto, aqui usamos série parcial de Fourier nas variáveis $\left(t^{\prime}, x\right)$, ou seja, faremos uso das séries formais

$$
u(t, x)=\sum_{(\kappa, \xi) \in \mathbb{Z}^{m} \times \mathbb{Z}} \hat{u}\left(t^{\prime \prime}, \kappa, \xi\right) e^{i\left(\kappa \cdot t^{\prime}+\xi x\right)}
$$

e

$$
h(t, x)=\sum_{(\kappa, \xi) \in \mathbb{Z}^{m} \times \mathbb{Z}} \hat{h}\left(t^{\prime \prime}, \kappa, \xi\right) e^{i\left(\kappa \cdot t^{\prime}+\xi x\right)} .
$$

Motivados por (2.25), substituímos as séries (2.26) e (2.27) na equação

$$
h=L_{n} L_{n-1} \ldots L_{1} u
$$


donde obtemos para cada $(\kappa, \xi) \in \mathbb{Z}^{m} \times \mathbb{Z}$ a seguinte igualdade

$$
\hat{h}\left(t^{\prime \prime}, \kappa, \xi\right)=\left(\prod_{j=m+1}^{n}\left(\partial_{t_{j}}+i \xi c_{j}\left(t_{j}\right)\right) \prod_{j=1}^{m} i\left(\kappa_{j}+\xi a_{j 0}\right)\right) \hat{u}\left(t^{\prime \prime}, \kappa, \xi\right),
$$

na qual $c_{j}\left(t_{j}\right)=a_{j 0}+i b_{j}\left(t_{j}\right), j=m+1, \ldots, n$.

Como $a_{0}^{\prime}=\left(a_{10}, \ldots, a_{m 0}\right) \notin \mathbb{Q}^{m}$ é Liouville, existe uma constante $C>0$ e uma sequência $\left\{\left(\kappa_{l}, \xi_{l}\right)\right\}$ em $\mathbb{Z}^{m} \times \mathbb{Z}, \xi_{l} \geq 2$, tal que

$$
\left|\xi_{l} a_{0}^{\prime}+\kappa_{l}\right| \doteq \max _{j=1, \ldots, m}\left|\xi_{l} a_{j 0}+\kappa_{l}^{(j)}\right|<C\left|\left(\kappa_{l}, \xi_{l}\right)\right|^{-l}, \forall l \in \mathbb{N} .
$$

$\operatorname{Logo}$, como $c_{j 0} \notin \mathbb{Q}, j=m+1, \ldots, n$, para cada $l \in \mathbb{N}$ a equação (2.28) possui uma única solução dada por

$$
\hat{u}\left(t^{\prime \prime}, \kappa_{l}, \xi_{l}\right)=\delta_{l} \int_{[0,2 \pi]^{n-m}} e^{i \xi_{l}\left(\mathcal{C}\left(t^{\prime \prime}-s^{\prime \prime}\right)-\mathcal{C}\left(t^{\prime \prime}\right)\right)} \hat{h}\left(t^{\prime \prime}-s^{\prime \prime}, \kappa_{l}, \xi_{l}\right) d s^{\prime \prime},
$$

sendo

$$
\delta_{l}=\prod_{j=1}^{m}\left[i\left(\kappa_{l}^{(j)}+\xi_{l} a_{j 0}\right)\right]^{-1} \prod_{j=m+1}^{n}\left[1-e^{-i 2 \pi \xi_{l} c_{j 0}}\right]^{-1} \quad \text { e } \quad \mathcal{C}\left(t^{\prime \prime}\right)=a_{0}^{\prime \prime} \cdot t^{\prime \prime}+i B\left(t^{\prime \prime}\right) .
$$

Seja $v\left(t^{\prime \prime}, \kappa_{l}, \xi_{l}\right)$ a expressão integral dada pelo lado direito de (2.30). Dessa forma definimos as funções $f_{j}$ como segue

$$
f_{j}(t, x)=\sum_{l \in \mathbb{N}} \hat{f}_{j}\left(t^{\prime \prime}, \kappa_{l}, \xi_{l}\right) e^{i\left(\kappa_{l} \cdot t^{\prime}+\xi_{l} x\right)}, \quad j=1, \ldots, n,
$$

nas quais para cada $l \in \mathbb{N}$ temos

$$
\hat{f}_{j}\left(t^{\prime \prime}, \kappa_{l}, \xi_{l}\right) \doteq \begin{cases}i\left(\kappa_{l}^{(j)}+\xi_{l} a_{j 0}\right) v\left(t^{\prime \prime}, \kappa_{l}, \xi_{l}\right) & \text { se } j=1, \ldots, m \\ \left(\frac{\partial}{\partial t_{j}}+i \xi_{l} c_{j}\left(t_{j}\right)\right) v\left(t^{\prime \prime}, \kappa_{l}, \xi_{l}\right) & \text { se } j=m+1, \ldots, n\end{cases}
$$

As funções $\hat{f}_{j}\left(\cdot, \kappa_{l}, \xi_{l}\right)$ ficam bem definidas pois dependem apenas da escolha de $\hat{h}\left(\cdot, \kappa_{l}, \xi_{l}\right)$. Obtemos dessa definição as seguintes fórmulas:

$$
\hat{f}_{j}\left(t^{\prime \prime}, \kappa_{l}, \xi_{l}\right)=i\left(\kappa_{l}^{(j)}+\xi_{l} a_{j 0}\right) \delta_{l} \int_{[0,2 \pi]^{n-m}} e^{i \xi_{l}\left(\mathcal{C}\left(t^{\prime \prime}-s^{\prime \prime}\right)-\mathcal{C}\left(t^{\prime \prime}\right)\right)} \hat{h}\left(t^{\prime \prime}-s^{\prime \prime}, \kappa_{l}, \xi_{l}\right) d s^{\prime \prime}
$$

para $j=1, \ldots, m$, e

$$
\hat{f}_{j}\left(t^{\prime \prime}, \kappa_{l}, \xi_{l}\right)=\frac{\delta_{l}}{d_{j \xi_{l}}} \int_{[0,2 \pi]^{n-m-1}} e^{i \xi_{l}\left(\mathcal{C}\left(t^{\prime \prime}-s^{\prime \prime}+s_{j} e_{j}\right)-\mathcal{C}\left(t^{\prime \prime}\right)\right)} \hat{h}\left(t^{\prime \prime}-s^{\prime \prime}+s_{j} e_{j}, \kappa_{l}, \xi_{l}\right) d s_{(j)}^{\prime \prime},
$$

para $j=m+1, \ldots, n$, sendo $d_{j \xi_{l}}=\left(1-e^{-i 2 \pi \xi_{l} c_{j 0} 0}\right)^{-1}$ e $d s_{(j)}^{\prime \prime}=d s_{m+1} \ldots d s_{j-1} d s_{j+1} \ldots d s_{n}$. 


\section{Construção da função $h$}

Denotamos por $M$ o máximo da função $B=B\left(t^{\prime \prime}\right)$ sobre o cubo $[0,2 \pi]_{t^{\prime \prime}}^{n-m}$ e por $M_{j}$ o máximo de $B$ sobre $[0,2 \pi]_{t^{\prime \prime}}^{n-m} \cap\left\{t_{j}=0\right\}, j=m+1, \ldots, n$. Logo, pelas argumentações feitas anteriormente temos $0 \leq \widetilde{M}<M$ sendo $\widetilde{M}$ o maior $M_{j} \operatorname{com} j=m+1, \ldots, n$.

A partir da sequência $\left\{\left(\kappa_{l}, \xi_{l}\right)\right\}$ que obtemos por $a_{0}^{\prime}$ ser Liouville e que satisfaz (2.29), definimos os conjuntos

$$
\begin{aligned}
& \mathcal{F}_{1}=\left\{l \in \mathbb{N} ; \quad e^{\xi_{l} \frac{M-\widetilde{M}}{2}}\left|\kappa_{l}+\xi_{l} a_{0}^{\prime}\right| \leq 1\right\}, \\
& \mathcal{F}_{2}=\left\{l \in \mathbb{N} ; \quad e^{\xi_{l} \frac{M-\widetilde{M}}{2}}\left|\kappa_{l}+\xi_{l} a_{0}^{\prime}\right|>1\right\} .
\end{aligned}
$$

Com base nesses conjuntos definimos a sequência $\left(\gamma_{l}\right)_{l \in \mathbb{N}}$ dada por

$$
\gamma_{l}=\left\{\begin{array}{ll}
e^{\lambda \xi_{l}} & \text { se } l \in \mathcal{F}_{1} \\
\left|\kappa_{l}+\xi_{l} a_{0}^{\prime}\right|^{-1 / 2} & \text { se } l \in \mathcal{F}_{2}
\end{array},\right.
$$

na qual $0<\lambda<(M-\widetilde{M}) / 2$ é uma constante.

Sejam $0<\delta<\pi / 2$ e $\mathcal{Q}_{\delta}=\left\{t^{\prime \prime} \in \mathbb{R}^{n-m},\left|t^{\prime \prime}\right|<\delta\right\}$. Considere então uma função de corte $\chi_{\delta} \in C_{c}^{\infty}\left(\mathbb{R}^{n-m}\right)$ tal que

$$
\chi_{\delta}\left(t^{\prime \prime}\right)= \begin{cases}1 & \text { se } t^{\prime \prime} \in \mathcal{Q}_{\delta} \\ 0 & \text { se } t^{\prime \prime} \notin \mathcal{Q}_{2 \delta}\end{cases}
$$

e $0 \leq \chi_{\delta}\left(t^{\prime \prime}\right) \leq 1$, para todo $t^{\prime \prime} \in \mathbb{R}^{n-m}$.

Utilizando-se da função $\chi_{\delta}$, definimos três funções periódicas suaves que serão fundamentais na posterior definição de $h$. Seja $t_{*}^{\prime \prime} \in(0,2 \pi)^{n-m}$ tal que $B\left(t_{*}^{\prime \prime}\right)=M$, então definimos

$$
\begin{gathered}
p\left(t^{\prime \prime}\right)=\sum_{\nu \in \mathbb{Z}^{n-m}} a_{0}^{\prime \prime} \cdot\left(t_{*}^{\prime \prime}-t^{\prime \prime}-2 \pi \nu\right) \chi_{\delta}\left(t^{\prime \prime}+2 \pi \nu\right), \\
q\left(t^{\prime \prime}\right)=1+\sum_{\nu \in \mathbb{Z}^{n-m}}\left(\left|t^{\prime \prime}+2 \pi \nu\right|^{2}-1\right) \chi_{\delta}\left(t^{\prime \prime}+2 \pi \nu\right), \\
r\left(t^{\prime \prime}\right)=\sum_{\nu \in \mathbb{Z}^{n-m}} B\left(t^{\prime \prime}+2 \pi \nu\right) \chi_{\delta}\left(t^{\prime \prime}+2 \pi \nu\right) .
\end{gathered}
$$

As funções $p, q$ e $r$ possuem as seguintes propriedades:

- $q\left(t^{\prime \prime}\right) \geq 0, \quad \forall t^{\prime \prime} \in \mathbb{R}^{n-m}$ e $q\left(t^{\prime \prime}\right)=0$ se, e somente se, $t^{\prime \prime}=2 \pi \nu, \nu \in \mathbb{Z}^{n-m}$.

- $r(2 \pi \nu)=0, \forall \nu \in \mathbb{Z}^{n-m}$.

- Se $\left|t^{\prime \prime}\right|<\delta$, temos $p\left(t^{\prime \prime}\right)=a_{0}^{\prime \prime} \cdot\left(t_{*}^{\prime \prime}-t^{\prime \prime}\right), q\left(t^{\prime \prime}\right)=\left|t^{\prime \prime}\right|^{2}$ e $r\left(t^{\prime \prime}\right)=B\left(t^{\prime \prime}\right)$. 
Finalmente definimos

$$
h(t, x)=\sum_{l \in \mathbb{N}} \hat{h}\left(t^{\prime \prime}, \kappa_{l}, \xi_{l}\right) e^{i\left(\kappa_{l} \cdot t^{\prime}+\xi_{l} x\right)},
$$

sendo $\hat{h}\left(\cdot, \kappa_{l}, \xi_{l}\right)$ os coeficientes dados por

$$
\hat{h}\left(t^{\prime \prime}, \kappa_{l}, \xi_{l}\right)=\frac{\gamma_{l}}{\delta_{l}} e^{\xi_{l}\left(-K q\left(t^{\prime \prime}\right)+r\left(t^{\prime \prime}\right)-M+i p\left(t^{\prime \prime}\right)\right)} .
$$

Proposição 2.15. Para $K>0$ suficientemente grade, $h \in C^{\infty}\left(\mathbb{T}^{n+1}\right)$.

Demonstração. Seja $\phi\left(t^{\prime \prime}\right)=-K q\left(t^{\prime \prime}\right)+r\left(t^{\prime \prime}\right)-M$, então $\phi(2 \nu \pi)=-M$ para todo $\nu \in \mathbb{Z}^{n-m}$. Como $\phi$ é contínua, existe uma vizinhança $U$ dos vértices do cubo $[0,2 \pi]^{n-m}$ tal que

$$
\phi\left(t^{\prime \prime}\right)<-M+\lambda, \quad \forall t^{\prime \prime} \in U .
$$

Considere $\sigma \doteq \min \left\{q\left(t^{\prime \prime}\right) ; \quad t^{\prime \prime} \in[0,2 \pi]^{n-m} \backslash U\right\}$. Uma vez que a função $q$ é não negativa e, restrita ao cubo $[0,2 \pi]^{n-m}$, anula-se apenas nos vértices, segue que $\sigma>0$. Logo, escolhendo $K>(\eta-\lambda) / \sigma \operatorname{com} \eta \doteq \max \left\{r\left(t^{\prime \prime}\right) ; t^{\prime \prime} \in[0,2 \pi]^{n-m} \backslash U\right\}$ obtemos

$$
\phi\left(t^{\prime \prime}\right)<-M+\lambda, \quad \forall t^{\prime \prime} \in[0,2 \pi]^{n-m} \backslash U .
$$

Por outro lado, fazendo uso do Lema 2.6, existe uma constante $C>0$ tal que

$$
\frac{1}{\left|\delta_{l}\right|} \leq C\left|\kappa_{l}+\xi_{l} a_{0}^{\prime}\right|^{m}, \quad \forall l \in \mathbb{N}
$$

Portanto, segue da definição dos coeficientes $\hat{h}\left(\cdot, \kappa_{l}, \xi_{l}\right)$ em $(2.34)$ a seguinte desigualdade

$$
\begin{aligned}
\left|\hat{h}\left(t^{\prime \prime}, \kappa_{l}, \xi_{l}\right)\right| & \leq C \gamma_{l}\left|\kappa_{l}+\xi_{l} a_{0}^{\prime}\right|^{m} e^{-\xi_{l}(M-\lambda)} \\
& =\left\{\begin{array}{ll}
C\left|\kappa_{l}+\xi_{l} a_{0}^{\prime}\right|^{m} e^{-\xi_{l}(M-2 \lambda)} & \text { se } l \in \mathcal{F}_{1} \\
C\left|\kappa_{l}+\xi_{l} a_{0}^{\prime}\right|^{(2 m-1) / 2} e^{-\xi_{l}(M-\lambda)} & \text { se } l \in \mathcal{F}_{2}
\end{array} .\right.
\end{aligned}
$$

Estimativas similares para as derivadas de $\hat{h}\left(\cdot, \kappa_{l}, \xi_{l}\right)$ garantem que $h \in C^{\infty}\left(\mathbb{T}^{n+1}\right)$.

Vejamos como ficam as fórmulas (2.30), (2.31) e (2.32) considerando os coeficientes $\hat{h}(\cdot, \kappa, \xi)$ definidos em $(2.34)$.

$$
\begin{gathered}
\hat{u}\left(t^{\prime \prime}, \kappa_{l}, \xi_{l}\right)=\gamma_{l} \int_{[0,2 \pi]^{n-m}} e^{\xi_{l}\left(\varphi_{1}\left(t^{\prime \prime}, s^{\prime \prime}\right)+i \varphi_{2}\left(t^{\prime \prime}, s^{\prime \prime}\right)\right)} d s^{\prime \prime}, \\
\hat{f}_{j}\left(t^{\prime \prime}, \kappa_{l}, \xi_{l}\right)=i\left(\kappa_{l}^{(j)}+\xi_{l} a_{j 0}\right) \gamma_{l} \int_{[0,2 \pi]^{n-m}} e^{\xi_{l}\left(\varphi_{1}\left(t^{\prime \prime}, s^{\prime \prime}\right)+i \varphi_{2}\left(t^{\prime \prime}, s^{\prime \prime}\right)\right)} d s^{\prime \prime},
\end{gathered}
$$


para $j=1, \ldots, m$,

$$
\hat{f}_{j}\left(t^{\prime \prime}, \kappa_{l}, \xi_{l}\right)=\frac{\gamma_{l}}{d_{j \xi_{l}}} \int_{[0,2 \pi]^{n-m-1}} e^{\xi_{l}\left(\varphi_{1}\left(t^{\prime \prime}, s^{\prime \prime}-s_{j} e_{j}\right)+i \varphi_{2}\left(t^{\prime \prime}, s^{\prime \prime}-s_{j} e_{j}\right)\right)} d s_{(j)}^{\prime \prime},
$$

para $j=m+1, \ldots, n$. Sendo

$$
\varphi_{1}\left(t^{\prime \prime}, s^{\prime \prime}\right)=B\left(t^{\prime \prime}\right)-B\left(t^{\prime \prime}-s^{\prime \prime}\right)-K q\left(t^{\prime \prime}-s^{\prime \prime}\right)+r\left(t^{\prime \prime}-s^{\prime \prime}\right)-M
$$

$\mathrm{e}$

$$
\varphi_{2}\left(t^{\prime \prime}, s^{\prime \prime}\right)=p\left(t^{\prime \prime}-s^{\prime \prime}\right)-a_{0}^{\prime \prime} \cdot s^{\prime \prime}
$$

funções a valores reais.

Proposição 2.16. Para $K>0$ suficientemente grande, $f_{j} \in C^{\infty}\left(\mathbb{T}^{n+1}\right), j=1, \ldots, m$.

Demonstração. Afirmamos que $\varphi_{1}\left(t^{\prime \prime}, s^{\prime \prime}\right) \leq 0$, para todo $t^{\prime \prime}, s^{\prime \prime} \in[0,2 \pi]^{n-m}$. Com efeito, denotamos por $\mathcal{F}$ a fronteira do cubo $[0,2 \pi]^{n-m}$. Como $B<M$ sobre $\mathcal{F}$, existe $0<\delta_{0} \leq \delta$ tal que $B \leq M$ sobre o aberto $U=\left\{\tau^{\prime \prime} \in \mathbb{R}^{n-m} ; \operatorname{dist}\left(\tau^{\prime \prime}, \mathcal{F}\right)<\delta_{0}\right\}$.

Para cada $2 \pi \nu, \nu \in \mathbb{Z}^{n-m}$, considere a bola aberta de raio $\delta_{0}$ centrada em $2 \pi \nu$. Denotamos por $V$ a união (disjunta) dessas bolas.

Dados $t^{\prime \prime}, s^{\prime \prime} \in[0,2 \pi]^{n-m}$ veja que a diferença $t^{\prime \prime}-s^{\prime \prime} \in[-2 \pi, 2 \pi]^{n-m}$.

Se $t^{\prime \prime}-s^{\prime \prime} \in V$, então $\left|\left(t^{\prime \prime}-s^{\prime \prime}\right)-2 \pi \nu\right|<\delta_{0}$ para algum $2 \pi \nu, \nu \in \mathbb{Z}^{n-m}$, logo $r\left(t^{\prime \prime}-s^{\prime \prime}\right)=r\left(t^{\prime \prime}-s^{\prime \prime}-2 \nu \pi\right)=B\left(t^{\prime \prime}-s^{\prime \prime}-2 \nu \pi\right) \mathrm{e}$

$$
\begin{aligned}
\varphi_{1}\left(t^{\prime \prime}, s^{\prime \prime}\right) & =B\left(t^{\prime \prime}\right)-B\left(t^{\prime \prime}-s^{\prime \prime}\right)-K q\left(t^{\prime \prime}-s^{\prime \prime}\right)+r\left(t^{\prime \prime}-s^{\prime \prime}\right)-M \\
& =B\left(t^{\prime \prime}\right)-B\left(t^{\prime \prime}-s^{\prime \prime}\right)-K q\left(t^{\prime \prime}-s^{\prime \prime}\right)+B\left(t^{\prime \prime}-s^{\prime \prime}-2 \nu \pi\right)-M \\
& =B\left(t^{\prime \prime}\right)-M-K q\left(t^{\prime \prime}-s^{\prime \prime}\right)-2 \pi \nu \cdot b_{0} \\
& =B\left(t^{\prime \prime}-2 \pi \nu\right)-M-K q\left(t^{\prime \prime}-s^{\prime \prime}\right) \leq B\left(t^{\prime \prime}-2 \pi \nu\right)-M .
\end{aligned}
$$

Observe na desigualdade anterior que, caso $\nu=0$ então $\varphi_{1}\left(t^{\prime \prime}, s^{\prime \prime}\right) \leq 0$. Caso $\nu \neq 0$ temos $\left(t^{\prime \prime}-2 \pi \nu\right) \notin(0,2 \pi)^{n-m}$, então

$$
\operatorname{dist}\left(t^{\prime \prime}-2 \pi \nu, \mathcal{F}\right) \leq \operatorname{dist}\left(t^{\prime \prime}-2 \pi \nu, s^{\prime \prime}\right)=\operatorname{dist}\left(t^{\prime \prime}-s^{\prime \prime}, 2 \pi \nu\right)<\delta_{0},
$$

$\log 0 t^{\prime \prime}-2 \pi \nu \in U$ e $\varphi_{1}\left(t^{\prime \prime}, s^{\prime \prime}\right) \leq 0$.

Se $t^{\prime \prime}-s^{\prime \prime} \notin V$ então

$$
\begin{aligned}
\varphi_{1}\left(t^{\prime \prime}, s^{\prime \prime}\right) & \leq-B\left(t^{\prime \prime}-s^{\prime \prime}\right)-K q\left(t^{\prime \prime}-s^{\prime \prime}\right)+r\left(t^{\prime \prime}-s^{\prime \prime}\right) \\
& \leq C-K \sigma,
\end{aligned}
$$

sendo

$$
C \doteq \max \left\{r\left(\tau^{\prime \prime}\right)-B\left(\tau^{\prime \prime}\right) ; \tau^{\prime \prime} \in[-2 \pi, 2 \pi]^{n-m} \backslash V\right\}
$$


e

$$
0<\sigma \doteq \min \left\{q\left(\tau^{\prime \prime}\right) ; \quad \tau^{\prime \prime} \in[-2 \pi, 2 \pi]^{n-m} \backslash V\right\}
$$

Assim, escolhendo $K>0$ tal que $C \leq K \sigma$, concluímos a afirmação feita no início da demonstração. Obtemos assim a seguinte estimativa para as funções $\hat{f}_{j}\left(t^{\prime \prime}, \kappa, \xi\right)$ dadas em (2.36),

$$
\begin{aligned}
\left|\hat{f}_{j}\left(t^{\prime \prime}, \kappa_{l}, \xi_{l}\right)\right| & \leq(2 \pi)^{n-m} \gamma_{l}\left|\kappa_{l}+\xi_{l} a_{0}^{\prime}\right| \\
& \leq(2 \pi)^{n-m}\left\{\begin{array}{ll}
e^{-\xi_{l}\left(\frac{M-\widetilde{M}}{2}-\lambda\right)} & \text { se } l \in \mathcal{F}_{1} \\
\left|\kappa_{l}+\xi_{l} a_{0}^{\prime}\right|^{1 / 2} & \text { se } l \in \mathcal{F}_{2}
\end{array} .\right.
\end{aligned}
$$

Vejamos que uma estimativa semelhante ocorre para as derivadas de $\hat{f}_{j}\left(t^{\prime \prime}, \kappa, \xi\right)$. De fato, dado $\alpha \in \mathbb{Z}_{+}^{n-m}$ temos

$$
\partial^{\alpha} \hat{f}_{j}\left(t^{\prime \prime}, \kappa_{l}, \xi_{l}\right)=i\left(\kappa_{l}^{(j)}+\xi_{l} a_{j 0}\right) \gamma_{l} \int_{[0,2 \pi]^{m-n}} P\left(t^{\prime \prime}, s^{\prime \prime}, \xi_{l}\right) e^{\xi_{l} \varphi\left(t^{\prime \prime}, s^{\prime \prime}\right)} d s^{\prime \prime}
$$

sendo $\varphi \doteq \varphi_{1}+i \varphi_{2}$ e $P$ um polinômio envolvendo potências de $\xi_{l}$ (com grau menor ou igual a $|\alpha|)$ e derivadas parciais de $\varphi$, as quais são limitadas em $[0,2 \pi]^{2(n-m)}$. Então, existe uma constante $C_{\alpha}>0$ tal que

$$
\left|P\left(t^{\prime \prime}, s^{\prime \prime}, \xi_{l}\right)\right| \leq C_{\alpha} \xi_{l}^{|\alpha|}, \quad \forall t^{\prime \prime}, s^{\prime \prime} \in[0,2 \pi]^{n-m}
$$

Assim,

$$
\begin{aligned}
\left|\partial^{\alpha} \hat{f}_{j}\left(t^{\prime \prime}, \kappa_{l}, \xi_{l}\right)\right| & \leq \gamma_{l}\left|\kappa_{l}+\xi_{l} a_{0}^{\prime}\right| \int_{[0,2 \pi]^{n-m}}\left|P\left(t^{\prime \prime}, s^{\prime \prime}, \xi_{l}\right)\right|\left|e^{\xi_{l} \varphi\left(t^{\prime \prime}, s^{\prime \prime}\right)}\right| d s^{\prime \prime} \\
& \leq(2 \pi)^{n-m} C_{\alpha} \xi_{l}^{|\alpha|}\left|\kappa_{l}+\xi_{l} a_{0}^{\prime}\right| \gamma_{l} \\
& \leq(2 \pi)^{n-m} C_{\alpha} \xi_{l}^{|\alpha|} \begin{cases}e^{-\xi_{l}\left(\frac{M-\widetilde{M}}{2}-\lambda\right)} & \text { se } l \in \mathcal{F}_{1} \\
\left|\kappa_{l}+\xi_{l} a_{0}^{\prime}\right|^{1 / 2} & \text { se } l \in \mathcal{F}_{2}\end{cases}
\end{aligned}
$$

e segue que $f_{j} \in C^{\infty}\left(\mathbb{T}^{n+1}\right), j=1, \ldots, m$.

Proposição 2.17. Para $K>0$ suficientemente grande, $f_{j} \in C^{\infty}\left(\mathbb{T}^{n+1}\right)$ para $j=$ $m+1, \ldots, n$. 
Demonstração. Fixamos um $j \in\{s+1, \ldots, n\}$ e identificamos os pontos $s^{\prime \prime}-s_{j} e_{j}$ $\operatorname{com}\left(s_{m+1}, \ldots, s_{j-1}, s_{j+1}, \ldots, s_{n}\right) \in \mathbb{R}^{n-m-1}$. Afirmamos que $\varphi_{1}\left(t^{\prime \prime}, s^{\prime \prime}-s_{j} e_{j}\right) \leq$ $(\widetilde{M}-M) / 2$, para todo $t^{\prime \prime}, s^{\prime \prime} \in[0,2 \pi]^{n-m}$. Com efeito, considere o conjunto

$$
\mathcal{Q}_{j}=\left\{\left(t^{\prime \prime}, s^{\prime \prime}-s_{j} e_{j}\right) \in[0,2 \pi]^{n-m} \times[0,2 \pi]^{n-m-1} ; q\left(t^{\prime \prime}-s^{\prime \prime}+s_{j} e_{j}\right)=0\right\} .
$$

Note que o conjunto $\mathcal{Q}_{j}$ é fechado uma vez que a função $q$ é contínua. Além disso $\left(t^{\prime \prime}, s^{\prime \prime}-s_{j} e_{j}\right) \in \mathcal{Q}_{j}$ se, e somente se, $t^{\prime \prime}-s^{\prime \prime}+s_{j} e_{j}=2 \nu \pi \in[0,2 \pi]^{n-m} \operatorname{com} \nu \in \mathbb{Z}^{n-m}$. Portanto,

$$
\begin{aligned}
\varphi_{1}\left(t^{\prime \prime}, s^{\prime \prime}-s_{j} e_{j}\right) & =B\left(t^{\prime \prime}\right)-B\left(t^{\prime \prime}-s^{\prime \prime}+s_{j} e_{j}\right)-r\left(t^{\prime \prime}-s^{\prime \prime}+s_{j} e_{j}\right)-M \\
& =B\left(s^{\prime \prime}-s_{j} e_{j}+2 \nu \pi\right)-B(2 \nu \pi)+r(2 \nu \pi)-M \\
& =B\left(s^{\prime \prime}-s_{j} e_{j}\right)-M \\
& \leq M_{j}-M<\frac{\widetilde{M}-M}{2}, \quad\left(t^{\prime \prime}, s^{\prime \prime}-s_{j} e_{j}\right) \in \mathcal{Q}_{j} .
\end{aligned}
$$

Como $\mathcal{Q}_{j}$ é fechado existe um aberto $U \supset \mathcal{Q}_{j}$ tal que

$$
\varphi_{1}\left(t^{\prime \prime}, s^{\prime \prime}-s_{j} e_{j}\right) \leq \frac{\widetilde{M}-M}{2}, \quad\left(t^{\prime \prime}, s^{\prime \prime}-s_{j} e_{j}\right) \in U .
$$

Sejam

$$
\sigma \doteq \min \left\{q\left(t^{\prime \prime}-s^{\prime \prime}+s_{j} e_{j}\right) ;\left(t^{\prime \prime}, s^{\prime \prime}-s_{j} e_{j}\right) \in \mathcal{K}\right\}
$$

e

$$
\mu \doteq \min \left\{B\left(t^{\prime \prime}-s^{\prime \prime}+s_{j} e_{j}\right)+r\left(t^{\prime \prime}-s^{\prime \prime}+s_{j} e_{j}\right) ; \quad\left(t^{\prime \prime}, s^{\prime \prime}-s_{j} e_{j}\right) \in \mathcal{K}\right\}
$$

sendo $\mathcal{K}$ o compacto $\left([0,2 \pi]^{n-m} \times[0,2 \pi]^{n-m-1}\right) \backslash U$. Dessa forma, $\sigma>0$ e escolhendo $K$ positivo tal que $K \geq\left(\frac{M-\widetilde{M}}{2}-\mu\right) / \sigma$ temos

$$
\varphi_{1}\left(t^{\prime \prime}, s^{\prime \prime}-s_{j} e_{j}\right) \leq-\mu-K \sigma \leq \frac{\widetilde{M}-M}{2}, \quad\left(t^{\prime \prime}, s^{\prime \prime}-s_{j} e_{j}\right) \in \mathcal{K},
$$

concluindo a afirmação feita.

Segue a seguinte estimativa para os coeficientes $\hat{f}_{j}\left(\cdot, \kappa_{l}, \xi_{l}\right)$ dados em $(2.37)$ :

$$
\begin{aligned}
\left|\hat{f}_{j}\left(t^{\prime \prime}, \kappa_{l}, \xi_{l}\right)\right| \leq C \gamma_{l} e^{-\xi_{l} \frac{M-\widetilde{M}}{2}} & = \begin{cases}C e^{-\xi_{l}\left(\frac{M-\widetilde{M}}{2}-\lambda\right)} & \text { se } l \in \mathcal{F}_{1} \\
C\left|\kappa_{l}+\xi_{l} a_{0}^{\prime}\right|^{-1 / 2} e^{-\xi_{l} \frac{M-\widetilde{M}}{2}} & \text { se } l \in \mathcal{F}_{2}\end{cases} \\
& \leq \begin{cases}C e^{-\xi_{l}\left(\frac{M-\widetilde{M}}{2}-\lambda\right)} & \text { se } l \in \mathcal{F}_{1} \\
C e^{-\xi_{l}\left(\frac{M-\widetilde{M}}{4}\right)} & \text { se } l \in \mathcal{F}_{2},\end{cases}
\end{aligned}
$$

sendo $C>0$ uma constante que não depende de $t^{\prime \prime}$ e $\left(\kappa_{l}, \xi_{l}\right)$. É possível obter estimativas semelhantes para as derivadas de $\hat{f}_{j}\left(\cdot, \kappa_{l}, \xi_{l}\right)$ como feito na Proposição 2.16 . Concluímos dessa forma que $f_{m+1}, \ldots, f_{n}$ são funções suaves. 
Proposição 2.18. Para $K>0$ suficientemente grande, não existe distribuição $u \in$ $\mathcal{D}^{\prime}\left(\mathbb{T}^{n+1}\right)$ cujos coeficientes parciais de Fourier nas variáveis $t_{1}, \ldots, t_{m}, x$, sejam os dados em (2.35).

Demonstração. Seja $t_{*}^{\prime \prime} \in(0,2 \pi)^{n-m}$ tal que $B\left(t_{*}^{\prime \prime}\right)=M$. Escrevemos

$$
\hat{u}\left(t_{*}^{\prime \prime}, \kappa_{l}, \xi_{l}\right)=\gamma_{l}\left(I_{l}+J_{l}\right)
$$

sendo

$$
\begin{aligned}
& I_{l}=\int_{\left|t_{*}^{\prime \prime}-s^{\prime \prime}\right|<\delta} e^{\xi_{l}\left(\varphi_{1}\left(t_{*}^{\prime \prime}, s^{\prime \prime}\right)+i \varphi_{2}\left(t_{*}^{\prime \prime}-s^{\prime \prime}\right)\right)} d s^{\prime \prime}, \\
& J_{l}=\int_{\left|t_{*}^{\prime \prime}-s^{\prime \prime}\right| \geq \delta} e^{\xi_{l}\left(\varphi_{1}\left(t_{*}^{\prime \prime}, s^{\prime \prime}\right)+i \varphi_{2}\left(t_{*}^{\prime \prime}-s^{\prime \prime}\right)\right)} d s^{\prime \prime} .
\end{aligned}
$$

Se $\left|t_{*}^{\prime \prime}-s^{\prime \prime}\right|<\delta$ temos $p\left(t_{*}^{\prime \prime}-s^{\prime \prime}\right)=a_{0}^{\prime \prime} \cdot\left(t_{*}^{\prime \prime}-\left(t_{*}^{\prime \prime}-s^{\prime \prime}\right)\right)=a_{0}^{\prime \prime} \cdot s^{\prime \prime}, q\left(t_{*}^{\prime \prime}-s^{\prime \prime}\right)=\left|t_{*}^{\prime \prime}-s^{\prime \prime}\right|^{2}$ e $r\left(t_{*}^{\prime \prime}-s^{\prime \prime}\right)=B\left(t_{*}^{\prime \prime}-s^{\prime \prime}\right)$. Logo, fazendo a mudança de variáveis $\xi_{l} K\left(t_{*}^{\prime \prime}-s^{\prime \prime}\right)=\sigma$ chegamos à seguinte igualdade

$$
\begin{aligned}
I_{l} & =\int_{\left|t_{*}^{\prime \prime}-s^{\prime \prime}\right|<\delta} e^{\xi_{l} \varphi_{1}\left(t_{*}^{\prime \prime}, s^{\prime \prime}\right)} e^{i \xi_{l} \varphi_{2}\left(t_{*}^{\prime \prime}-s^{\prime \prime}\right)} d s^{\prime \prime} \\
& =\int_{\left|t_{*}^{\prime \prime}-s^{\prime \prime}\right|<\delta} e^{-K \xi_{l}\left|t_{*}^{\prime \prime}-s^{\prime \prime}\right|^{2}} d s^{\prime \prime} \\
& =\frac{1}{\left(K \xi_{l}\right)^{(n-m) / 2}} \int_{|\sigma|<\delta \sqrt{K \xi_{l}}} e^{-|\sigma|^{2}} d \sigma=\frac{1}{\left(K \xi_{l}\right)^{(n-m) / 2}} \mu_{\xi_{l}}
\end{aligned}
$$

na qual $\mu_{\xi_{l}} \doteq \int_{|\sigma|<\delta \sqrt{K \xi_{l}}} e^{-|\sigma|^{2}} d \sigma$ é uma sequência de números reais positivos a qual é crescente e limitada superiormente.

Por outro lado, temos $q(\tau)>0$ para todo $\tau \in Q^{*}=t^{*}-[0,2 \pi]^{n-m}$. Portanto, a constante $\sigma=\min \left\{q(\tau) ; \tau \in Q^{*}\right.$ e $\left.|\tau| \geq \delta\right\}$ é positiva.

Para $K>0$ suficientemente grande obtemos a desigualdade

$$
\varphi\left(t_{*}^{\prime \prime}, s^{\prime \prime}\right) \leq-K \sigma+r\left(t_{*}^{\prime \prime}-s^{\prime \prime}\right)-B\left(t_{*}^{\prime \prime}-s^{\prime \prime}\right)<-\lambda, \forall s^{\prime \prime} \in[0,2 \pi]^{n-m} .
$$

Portanto,

$$
\left|J_{l}\right| \leq \int_{\left|t_{*}^{\prime \prime}-s^{\prime \prime}\right| \geq \delta} e^{\xi_{l} \varphi_{1}\left(t_{*}^{\prime \prime}, s^{\prime \prime}\right)} d s^{\prime \prime} \leq(2 \pi)^{n-m} e^{-\xi_{l} \lambda} .
$$

Logo, segue que

$$
\begin{aligned}
\left|\hat{u}\left(t_{*}^{\prime \prime}, \kappa_{l}, \xi_{l}\right)\right| & \geq \gamma_{l}\left(\left|I_{l}\right|-\left|J_{l}\right|\right) \\
& \geq \gamma_{l}\left(\frac{1}{\left(K \xi_{l}\right)^{(n-m) / 2}} \mu_{l}-(2 \pi)^{n-m} e^{-\xi_{l} \lambda}\right) .
\end{aligned}
$$


Agora, tomamos $N \in \mathbb{N}$ tal que $\frac{e^{\xi_{l} \lambda} \mu_{1}}{(2 \pi)^{(n-m)}\left(K \xi_{l}\right)^{(n-m) / 2}} \geq 1$ para todo $\xi_{l}>N$. Assim, da desigualdade anterior obtemos

$$
\begin{aligned}
\left|\hat{u}\left(t_{*}^{\prime \prime}, \kappa_{l}, \xi_{l}\right)\right| & \geq \gamma_{l}\left(\frac{1}{\left(K \xi_{l}\right)^{(n-m) / 2}} \mu_{l}-(2 \pi)^{n-m} e^{-\xi_{l} \lambda}\right) \\
& \geq \gamma_{l}\left(\frac{1}{\left(K \xi_{l}\right)^{(n-m) / 2}} \mu_{l}-\frac{1}{\left(K \xi_{l}\right)^{(n-m) / 2}} \mu_{1}\right) \\
& =\gamma_{l}\left(\mu_{l}-\mu_{1}\right) \frac{1}{\left(K \xi_{l}\right)^{(n-m) / 2}} \\
& \geq \frac{\left(\mu_{l}-\mu_{1}\right) \gamma_{l}}{\left(K \xi_{l}\right)^{(n-m) / 2}}, \quad \forall \xi_{l}>N .
\end{aligned}
$$

Portanto, pela forma que foi definido $\left(\gamma_{l}\right)_{l \in \mathbb{N}}$ em (2.33), concluímos que a sequência $\{\hat{u}(\cdot, \kappa, \xi)\}$ não pode ser uma sequência de coeficientes parciais de Fourier de nenhuma distribuição $u \in \mathcal{D}^{\prime}\left(\mathbb{T}^{n+1}\right)$.

Concluiremos agora a demonstração no caso 1.2 supondo que $\mathbb{Q} \cap\left\{c_{(m+1) 0}, \ldots\right.$, $\left.c_{n 0}\right\} \neq \emptyset$, lembrando que ainda estamos trabalhando com a hipótese $c_{0}^{\prime \prime} \notin \mathbb{Q}^{n-m}$.

Podemos assumir que $c_{(m+1) 0}, \ldots, c_{p 0} \notin \mathbb{Q}$ e que $c_{(p+1) 0}, \ldots, c_{n 0} \in \mathbb{Q}$ sendo $m+1 \leq$ $p<n$. Sejam $\mathbb{L}^{\#}$ o operador associado aos campos vetoriais $L_{1}, \ldots, L_{p}$ e $\mathcal{A}=\eta \mathbb{Z}$ o conjunto dos múltiplos de um $\eta \in \mathbb{N}$ que satisfaz $\eta\left(c_{(p+1) 0}, \ldots, c_{n 0}\right) \in \mathbb{Z}^{n-p}$.

Pelo Lema 1.7, para cada $l \in \mathbb{N}$ podemos assumir que $\xi_{l}, \kappa_{l}^{(j)} \in \mathcal{A}, j=1, \ldots, m$, na sequência $\left\{\left(\kappa_{l}, \xi_{l}\right)\right\}$ que satisfaz (2.29) considerada previamente. Assim, $\mathbb{L}_{\mathcal{A}}^{\#}$ não é globalmente resolúvel em $\mathbb{T}^{n-p+1}$ pela primeira parte do caso 1.2. Finalmente, pelo Lema 2.14 concluímos que $\mathbb{L}_{\mathcal{A}}$ não é globalmente resolúvel em $\mathbb{T}^{n+1}$.

\subsubsection{Caso 2: $a_{0}^{\prime} \in \mathbb{Q}^{m}$}

Uma vez que a condição $(i i)$ já não pode ser válida visto que $a_{0}^{\prime} \in \mathbb{Q}^{m}$, vamos supor que todas as funções $b_{j} \not \equiv 0$ mudam de sinal e alguma das condições exibidas em $($ iii) não está satisfeita. Separamos a demonstração em três partes.

Começamos assumindo que $c_{0}^{\prime \prime}=\left(c_{(m+1) 0}, \ldots, c_{n 0}\right) \notin \mathbb{Q}^{n-m}$, ou seja, a 1-forma $b$ não é exata ou $a_{0}^{\prime \prime} \notin \mathbb{Q}^{n-m}$. Reordenando os campos $L_{j}$, podemos assumir que $c_{(m+1) 0}, \ldots, c_{p 0} \in \mathbb{Q}$ e que $c_{(p+1) 0}, \ldots, c_{n 0} \notin \mathbb{Q}$ sendo $m \leq p \leq n-1(p=m$ significa $\left.c_{(m+1) 0}, \ldots, c_{n 0} \notin \mathbb{Q}\right)$.

Seja $\mathcal{A}=\eta \mathbb{Z}$ com $\eta \in \mathbb{N}$ tal que $\eta\left(c_{10}, \ldots, c_{p 0}\right) \in \mathbb{Z}^{p}$ e considere o operador $\mathbb{L}^{\#}$ associado aos campos vetoriais $L_{p+1}, \ldots, L_{n}$. Como $c_{(p+1) 0}, \ldots, c_{n 0} \notin \mathbb{Q}$, pela Proposição 2.3 concluímos que $\mathbb{L}^{\#}$ não é globalmente resolúvel em $\mathbb{T}^{n-p+1}$. Mais ainda, podemos afirmar que $\mathbb{L}_{\mathcal{A}}^{\#}$ não é globalmente resolúvel. De fato, lembremos que na construção 
de $f$ na Seção 2.2 usamos apenas frequências $\xi \in \mathbb{Z} \backslash \mathcal{G}$ sendo $\mathcal{G}$ definida em (2.12) a partir dos períodos $c_{j 0}$ racionais. Logo, como estamos assumindo $c_{(p+1) 0}, \ldots, c_{n 0} \notin \mathbb{Q}$ temos $\mathcal{G}=\emptyset$. Então, para o operador $\mathbb{L}^{\#}$, usando as mesmas ideias daquela seção, podemos construir $g$ usando apenas frequências em $\mathcal{A}$ tal que $\mathbb{L}_{\mathcal{A}}^{\#} v=g$ não possui solução $v \in \mathcal{D}^{\prime}\left(\mathbb{T}^{n-m+1}\right)$. Concluímos assim que $\mathbb{L}_{\mathcal{A}}^{\#}$ não é globalmente resolúvel em $\mathbb{T}^{n-p+1}$. Portanto, pelo Lema 2.14, o operador $\mathbb{L}_{\mathcal{A}}$ não é globalmente resolúvel em $\mathbb{T}^{n+1}$.

Suponhamos agora que $b$ é exata e $a_{0}^{\prime \prime} \in \mathbb{Q}^{n-m}$ (ou seja, $c_{0}^{\prime \prime}=\left(c_{(m+1) 0}, \ldots, c_{n 0}\right) \in$ $\left.\mathbb{Q}^{n-m}\right)$, porém $q_{J}<q_{*}$. Logo, existe ao menos um período $c_{j 0}, j=m+1, \ldots, n$, tal que $q_{J} c_{j 0} \notin \mathbb{Z}$. Assim, reordenando os campos se necessário, vamos assumir que $q_{J} a_{10}, \ldots, q_{J} a_{p 0} \in \mathbb{Z}$ e que $q_{J} a_{(p+1) 0}, \ldots, q_{J} a_{n 0} \notin \mathbb{Z}$ sendo $m \leq p \leq n-1$.

Escrevemos $a_{j 0}=r_{j} / s_{j}, j=p+1, \ldots, n$ com $r_{j} \in \mathbb{Z}$ e $s_{j} \in \mathbb{N}$ primos entre si. Nessa notação, definimos o conjunto

$$
\mathcal{G}=\bigcup_{j=p+1}^{n} s_{j} \mathbb{Z}_{+}
$$

Portanto, o conjunto $q_{J} \mathbb{Z}_{+} \backslash \mathcal{G}$ possui infinitos elementos.

Denotamos por $\mathbb{L}^{\#}$ o operador associado aos campos vetoriais $L_{p+1}, \ldots, L_{n}$ e por $\mathcal{A}$ o conjunto $q_{J} \mathbb{Z}$. Novamente, usando argumentos semelhantes aos feitos na Seção 2.2 podemos concluir que $\mathbb{L}_{\mathcal{A}}^{\#}$ não é globalmente resolúvel em $\mathbb{T}_{\left(t_{p+1}, \ldots, t_{n}, x\right)}^{n-p+1}$. Assim, pelo Lema 2.14 concluímos que $\mathbb{L}_{\mathcal{A}}$ não é globalmente resolúvel em $\mathbb{T}^{n+1}$.

Finalmente, suponha que $a_{0} \in \mathbb{Q}^{n}$, $b$ é exata e $q_{J}=q_{*}$, porém alguma primitiva global $B: \mathbb{T}^{n} \rightarrow \mathbb{R}$ da 1 -forma $b$ possui um subnível ou um supernível desconexo em $\mathbb{T}^{n}$.

Sejam $\mathcal{A}=q_{*} \mathbb{Z}$ e $\mathbb{L}_{0}=d_{t}+i b(t) \wedge \partial / \partial x$. O automorfismo do Lema 2.12 garante que $\mathbb{L}_{\mathcal{A}}$ é globalmente resolúvel se, e somente se, $\mathbb{L}_{0, \mathcal{A}}$ é globalmente resolúvel. Portanto, como $B$ possui um subnível ou um supernível desconexo em $\mathbb{T}^{n}$, o operador $\mathbb{L}_{0, \mathcal{A}}$ não é globalmente resolúvel pelo trabalho $[\mathrm{CH}]$ e, portanto, $\mathbb{L}$ não é globalmente resolúvel. 


$\frac{10}{3}$

\section{Resultados adicionais}

Sejam $b \in \bigwedge^{1} C^{\infty}\left(\mathbb{T}^{n} ; \mathbb{R}\right)$ uma 1-forma fechada e $\mathbb{L}$ o operador definido por

$$
\mathbb{L}=d_{t}+i b(t) \wedge \frac{\partial}{\partial x} .
$$

Pelo trabalho $[\mathrm{CH}]$, se $b$ for exata então o operador $\mathbb{L}$ será globalmente resolúvel se, e somente se, uma primitiva global de $b$ possuir todos subníveis e superníveis conexos em $\mathbb{T}^{n}$.

Quando a 1-forma $b$ não for exata, apresentaremos uma situação especial em que o operador $\mathbb{L}$ não é globalmente resolúvel. Usaremos isso para caracterizar a resolubilidade global em um caso parcialmente acoplado na Seção 3.3.

Daqui em diante vamos considerar $b$ não exata. Logo, se $r$ denota o posto do grupo de períodos $\operatorname{Per}(b)$ da 1 -forma $b$ e $s \doteq n-r$ o coposto de $\operatorname{Per}(b)$, então

$$
1 \leq r \leq n \quad \text { e } \quad 0 \leq s \leq n-1
$$

e pelo Lema 1.4 podemos assumir que

(i) $b_{10}=\cdots=b_{s 0}=0$ e $b_{n 0}<\cdots<b_{(s+1) 0}<0$, quando $s \geq 1$;

(ii) $b_{n 0}<\cdots<b_{10}<0$, quando $s=0$.

Usaremos a notação $t=\left(t^{\prime}, t^{\prime \prime}\right)$ para as coordenadas em $\mathbb{T}^{n}$, sendo $t^{\prime}=\left(t_{1}, \ldots, t_{s}\right)$ e $t^{\prime \prime}=\left(t_{s+1}, \ldots, t_{n}\right)$. Quando $s=0$, teremos apenas $t=t^{\prime \prime}=\left(t_{1}, \ldots, t_{n}\right)$. 
Capítulo 3 - Resultados adicionais

Seja $B: \mathbb{R}^{n} \rightarrow \mathbb{R}$ uma primitiva global do pull-back $\Pi^{*} b$ através do recobrimento universal $\Pi: \mathbb{R}^{n} \rightarrow \mathbb{T}^{n}$. Denotamos por $M$ o máximo da função $B$ sobre o cubo $[0,2 \pi]^{n}$ e por $M_{j}$ o máximo de $B$ sobre $[0,2 \pi]^{n} \cap\left\{t_{j}=0\right\}, j=1, \ldots, n$. Nessas condições vamos supor que:

( ) Para cada $j=s+1, \ldots, n$ temos $M_{j}<M$.

Observação: Essa condição significa que o máximo da função $B$ sobre o cubo $[0,2 \pi]^{n}$ não é atingido nas faces contidas nos planos $\left\{t_{j}=0\right\}, j=s+1, \ldots, n$, cujos períodos $b_{j 0} \neq 0$. E como os períodos $b_{(s+1) 0}, \ldots, b_{n 0}$ são negativos, o máximo de $B$ sobre $[0,2 \pi]^{n}$ também não será atingido nas faces paralelas contidas nos planos $\left\{t_{j}=2 \pi\right\}$, $j=s+1, \ldots, n$. No caso em que $s=0$, o máximo de $B$ sobre o cubo $[0,2 \pi]^{n}$ não ocorre na fronteira.

Podemos assumir que $B(0)=0$ considerando a primitiva $B-B(0)$. Dessa forma, se $\widetilde{M}$ é o maior $M_{j}, j=s+1, \ldots, n$, temos $\widetilde{M} \geq 0$ e $M-\widetilde{M}>0$.

\subsection{Um teorema de não resolubilidade}

Teorema 3.1. Seja $b=\sum_{j=1}^{n} b_{j}(t) d t_{j} \in \bigwedge^{1} C^{\infty}\left(\mathbb{T}^{n} ; \mathbb{R}\right)$ uma 1-forma fechada e não exata. Sob a condição $(\star)$ mencionada anteriormente o operador

$$
\mathbb{L}=d_{t}+i b(t) \wedge \frac{\partial}{\partial x}
$$

não é globalmente resolúvel em $\mathbb{T}^{n+1}$.

Demonstração. Vamos construir uma 1-forma $f=\sum_{j=1}^{n} f_{j}(t, x) d t_{j} \in \mathbb{E}$ de modo que a equação $\mathbb{L} u=f$ não possua solução $u \in \mathcal{D}^{\prime}\left(\mathbb{T}^{n+1}\right)$. Para esse operador $\mathbb{L}$ o espaço $\mathbb{E}$ é o seguinte:

$$
\mathbb{E}=\left\{f \in \bigwedge^{1} C^{\infty}\left(\mathbb{T}^{n+1}\right) ; \mathbb{L} f=0 \text { e } \int_{0}^{2 \pi} \hat{f}_{j}\left(0, \ldots, t_{j}, \ldots, 0,0\right) d t_{j}=0, j=1, \ldots, n\right\}
$$

Como queremos construir $f \in \mathbb{E}$ precisamos que $\mathbb{L} f=0$ ou equivalentemente $L_{j} f_{k}=L_{k} f_{j}, j, k=1, \ldots, n$. Esta condição juntamente com o fato do comutador $\left[L_{j}, L_{k}\right]$ ser nulo, resultam na seguinte igualdade

$$
L_{\sigma(s+1)} \ldots L_{\sigma(n-1)} L_{\sigma(n)} u=L_{s+1} \ldots L_{n-1} L_{n} u
$$

para qualquer permutação $\sigma$ dos inteiros $s+1, \ldots, n$. Ou seja, se a 1-forma $f \in \mathbb{E}$ e existe solução $u \in \mathcal{D}^{\prime}\left(\mathbb{T}^{n+1}\right)$ da equação $\mathbb{L} u=f$ então $u$ deve satisfazer (3.1). 
Vamos construir funções $f_{s+1}, \ldots, f_{n}$ motivados por (3.1) utilizando a seguinte equação

$$
h \doteq L_{s+1} \ldots L_{n-1} L_{n} u
$$

No caso em que $s \geq 1$, escolhemos as funções $f_{1}, \ldots, f_{s}$ identicamente nulas.

$$
\text { Construção das funções } f_{s+1}, \ldots, f_{n}
$$

Considere as séries parciais de Fourier de $u$ e $h$ definidas da seguinte forma

$$
u(t, x)=\sum_{\xi \in \mathbb{Z}} \hat{u}(t, \xi) e^{i \xi x} \quad \text { e } \quad h(t, x)=\sum_{\xi \in \mathbb{Z}} \hat{h}(t, \xi) e^{i \xi x} .
$$

Substituindo essas séries em (3.2), obtemos para cada $\xi \in \mathbb{Z}$ a seguinte equação

$$
\hat{h}(t, \xi)=\left(\frac{\partial}{\partial t_{s+1}}-\xi b_{s+1}(t)\right) \cdots\left(\frac{\partial}{\partial t_{n}}-\xi b_{n}(t)\right) \hat{u}(t, \xi) .
$$

Como $b_{j 0} \neq 0, j=s+1, \ldots, n$, para cada $\xi \neq 0$ a equação acima tem uma única solução dada por

$$
\hat{u}(t, \xi)=d_{\xi} \int_{[0,2 \pi]^{r}} e^{-\xi\left(B\left(t^{\prime}, t^{\prime \prime}-\sigma^{\prime \prime}\right)-B(t)\right)} \hat{h}\left(t^{\prime}, t^{\prime \prime}-\sigma^{\prime \prime}, \xi\right) d \sigma^{\prime \prime},
$$

sendo $d_{\xi}=d_{(s+1) \xi} \ldots d_{n \xi}, \operatorname{com} d_{j \xi}=\left(1-e^{2 \pi \xi b_{j 0}}\right)^{-1}$ e $d \sigma^{\prime \prime}=d \sigma_{s+1} \ldots d \sigma_{n}$.

As funções $\hat{h}(\cdot, \xi)$ serão construídas adiante e as funções $f_{s+1}, \ldots, f_{n}$ dependerão dessa construção. Desse modo, vamos obter uma 1-forma $f=\sum_{j=1}^{n} f_{j}(t, x) d t_{j} \in \mathbb{E}$ e caso exista solução $u$ da equação $\mathbb{L} u=f$ os coeficientes parciais de Fourier da distribuição $u$ serão dados por (3.3).

Definimos

$$
f_{j}(t, x)=\sum_{\xi=1}^{\infty} \hat{f}_{j}(t, \xi) e^{i \xi x}, \quad j=s+1, \ldots, n,
$$

sendo $\hat{f}_{j}(\cdot, \xi)$ dados como segue:

- Se $r=1$ temos $s+1=n$, neste caso

$$
\hat{f}_{n}(t, \xi)=\hat{h}(t, \xi), \quad \xi \in \mathbb{N} .
$$

- Se $r \geq 2$, definimos as funções $\hat{f}_{j}(\cdot, \xi)$ por

$$
\hat{f}_{j}(t, \xi)=\left(\frac{\partial}{\partial t_{j}}-\xi b_{j}(t)\right) v(t, \xi), \quad \xi \in \mathbb{N}, j=s+1, \ldots, n,
$$


sendo $v(\cdot, \xi)$ dada pelo lado direito de (3.3). Desenvolvendo o lado direito da igualdade acima chegamos a seguinte fórmula:

$$
\hat{f}_{j}(t, \xi)=\frac{d_{\xi}}{d_{j \xi}} \int_{[0,2 \pi]^{r-1}} e^{-\xi\left(B\left(t^{\prime}, t^{\prime \prime}-\sigma^{\prime \prime}+\sigma_{j} e_{j}\right)-B(t)\right)} \hat{h}\left(t^{\prime}, t^{\prime \prime}-\sigma^{\prime \prime}+\sigma_{j} e_{j}, \xi\right) d \sigma_{(j)}^{\prime \prime},
$$

na qual $d \sigma_{(j)}^{\prime \prime}=d \sigma_{s+1} \ldots d \sigma_{j-1} d \sigma_{j+1} \ldots d \sigma_{n}$ e $e_{j}=(0, \ldots, 1, \ldots, 0) \in \mathbb{R}_{t^{\prime \prime}}^{r}$.

As funções $\hat{f}_{j}(\cdot, \xi)$ estão bem definidas, pois só dependem da escolha das funções $\hat{h}(\cdot, \xi)$ que passamos a descrever agora.

\section{Construção da função $h$}

Sejam $0<\delta<\pi / 2$ e $\mathcal{Q}_{\delta}=\left\{t^{\prime \prime} \in \mathbb{R}^{r},\left|t^{\prime \prime}\right|<\delta\right\}$. Considere uma função de corte $\chi_{\delta} \in C_{c}^{\infty}\left(\mathbb{R}^{r}\right)$ tal que

$$
\chi_{\delta}\left(t^{\prime \prime}\right)= \begin{cases}1 & \text { se } t^{\prime \prime} \in \mathcal{Q}_{\delta} \\ 0 & \text { se } t^{\prime \prime} \notin \mathcal{Q}_{2 \delta}\end{cases}
$$

e $0 \leq \chi_{\delta}\left(t^{\prime \prime}\right) \leq 1$, para todo $t^{\prime \prime} \in \mathbb{R}^{r}$.

Usando a função $\chi_{\delta}$ definimos a função

$$
q\left(t^{\prime \prime}\right)=1+\sum_{\nu \in \mathbb{Z}^{r}}\left(\left|t^{\prime \prime}+2 \pi \nu\right|^{2}-1\right) \chi_{\delta}\left(t^{\prime \prime}+2 \pi \nu\right), \quad t^{\prime \prime} \in \mathbb{R}^{r}
$$

que possui as seguintes propriedades:

- $q$ é suave e $2 \pi$-periódica;

- $q \geq 0$ e $q\left(t^{\prime \prime}\right)=0$ se, e somente se, $t^{\prime \prime}=2 \pi \nu, \nu \in \mathbb{Z}^{r}$;

- Se $\left|t^{\prime \prime}\right|<\delta$ então $q\left(t^{\prime \prime}\right)=\left|t^{\prime \prime}\right|^{2}$.

Finalmente definimos a função $h$ da seguinte forma

$$
h(t, x)=\sum_{\xi=1}^{\infty} \hat{h}(t, \xi) e^{i \xi x},
$$

na qual

$$
\hat{h}(t, \xi)=e^{-\xi\left(B\left(0, t^{\prime \prime}\right)-B(t)+K q\left(t^{\prime \prime}\right)+M-\lambda\right)}, \quad \xi \in \mathbb{N},
$$

com $0<\lambda<(M-\widetilde{M}) / 2$ e $K>0$ constantes. A constante $K$ será suficientemente grande e escolhida posteriormente.

Proposição 3.2. Para $K>0$ suficientemente grande, $h \in C^{\infty}\left(\mathbb{T}^{n+1}\right)$. 
Demonstração. Seja

$$
\psi(t)=B(t)-B\left(0, t^{\prime \prime}\right)-K q\left(t^{\prime \prime}\right)-M+\lambda, \quad t \in \mathbb{R}^{n} .
$$

Afirmamos que $\psi(t)<(\widetilde{M}-M) / 2$ para todo $t \in[0,2 \pi]^{n}$. De fato, considere o conjunto

$$
Q=\left\{t^{\prime \prime} \in[0,2 \pi]^{r} ; q\left(t^{\prime \prime}\right)=0\right\}
$$

Logo, $t^{\prime \prime} \in Q$ se, e somente se, $t^{\prime \prime}$ é um vértice do cubo $[0,2 \pi]^{r}$, isto é, $t^{\prime \prime}=2 \pi \nu$ com $\nu=\left(\nu_{s+1}, \ldots, \nu_{n}\right)$ onde cada entrada $\nu_{j}$ é igual a 0 ou 1 . Portanto, para $t^{\prime \prime} \in Q$ temos

$$
\begin{aligned}
\psi\left(t^{\prime}, t^{\prime \prime}\right) & =B\left(t^{\prime}, 2 \pi \nu\right)-B(0,2 \pi \nu)-M+\lambda \\
& =B\left(t^{\prime}, 0\right)-M+\lambda \\
& \leq \widetilde{M}-M+\lambda \\
& <\frac{\widetilde{M}-M}{2}, \quad \forall\left(t^{\prime}, t^{\prime \prime}\right) \in[0,2 \pi]^{s} \times Q .
\end{aligned}
$$

Como $Q$ é um compacto, existe um aberto $U \supset[0,2 \pi]^{s} \times Q$ tal que

$$
\psi(t)<\frac{\widetilde{M}-M}{2}, \quad \forall t \in U
$$

Seja $\pi_{r}: \mathbb{R}^{n} \rightarrow \mathbb{R}^{r}$ dada por $\pi_{r}\left(t^{\prime}, t^{\prime \prime}\right)=t^{\prime \prime}$ e $\tilde{q}=q \circ \pi_{r}$. Assim, se $\mu$ denota o mínimo da função $\tilde{q}$ sobre o compacto $\mathcal{K}=[0,2 \pi]^{n} \backslash U$ temos $\mu>0$, caso contrário existiria $t \in \mathcal{K}$ tal que $0=\tilde{q}(t)=q\left(t^{\prime \prime}\right)$ o que implicaria $t^{\prime \prime} \in Q$ e portanto $t=\left(t^{\prime}, t^{\prime \prime}\right) \in$ $[0,2 \pi]^{s} \times Q \subset U$, o que é um absurdo.

Escolhemos $K>\left(\frac{M-\widetilde{M}}{2}-\eta+\lambda\right) / \mu$ sendo $\eta=\min \left\{B(t) ; t \in[-2 \pi, 2 \pi]^{n}\right\}$. Portanto,

$$
\begin{aligned}
\psi(t) & \leq-B\left(0, t^{\prime \prime}\right)-K q\left(t^{\prime \prime}\right)+\lambda \\
& =-B\left(0, t^{\prime \prime}\right)-K \tilde{q}(t)+\lambda \\
& \leq-\eta-K \mu+\lambda \\
& <\frac{\widetilde{M}-M}{2}, \quad \forall t \in \mathcal{K},
\end{aligned}
$$

concluindo a afirmação feita anteriormente.

A desigualdade acima juntamente com os coeficientes (3.6) resultam na seguinte estimativa,

$$
|\hat{h}(t, \xi)| \leq e^{-\xi \frac{M-\widetilde{M}}{2}}, \quad \forall t \in[0,2 \pi]^{n}, \forall \xi \in \mathbb{N} .
$$

Além disso, valem estimativas semelhantes para as derivadas $\partial^{\alpha} \hat{h}(\cdot, \xi), \alpha \in \mathbb{Z}_{+}^{n}$. 
Para cada $\xi \in \mathbb{N}$, vejamos como ficam as expressões dos coeficientes (3.3), (3.4) e (3.5) ao substituir os coeficientes definidos em (3.6).

- Qualquer que seja $1 \leq r \leq n$, temos

$$
\hat{u}(t, \xi)=d_{\xi} \int_{[0,2 \pi]^{r}} e^{-\xi\left(B\left(0, t^{\prime \prime}-\sigma^{\prime \prime}\right)-B(t)+K q\left(t^{\prime \prime}-\sigma^{\prime \prime}\right)+M-\lambda\right)} d \sigma^{\prime \prime} .
$$

- Se $r=1$, temos

$$
\hat{f}_{n}(t, \xi)=e^{-\xi\left(B\left(0, t_{n}\right)-B(t)+K q\left(t_{n}\right)+M-\lambda\right)}
$$

- Se $r \geq 2$, temos para cada $j=s+1, \ldots, n$

$$
\hat{f}_{j}(t, \xi)=\frac{d_{\xi}}{d_{j \xi}} \int_{[0,2 \pi]^{r-1}} e^{-\xi\left(B\left(0, t^{\prime \prime}-\sigma^{\prime \prime}+\sigma_{j} e_{j}\right)-B(t)+K q\left(t^{\prime \prime}-\sigma^{\prime \prime}+\sigma_{j} e_{j}\right)+M-\lambda\right)} d \sigma_{(j)}^{\prime \prime},
$$

Veja que $L_{k} f_{j}=L_{j} f_{k}$ para todo $j, k=1, \ldots, n$ e também vale

$$
\int_{0}^{2 \pi} \hat{f}_{j}\left(0, \ldots, t_{j}, \ldots, 0,0\right) d t_{j}=0
$$

pois $\hat{f}_{j}(\cdot, 0)=0$.

Proposição 3.3. $f_{s+1}, \ldots, f_{n} \in C^{\infty}\left(\mathbb{T}^{n+1}\right)$.

Demonstração. O caso em que $r=1(\operatorname{logo} s=n-1)$ se reduz à Proposição 3.2. Consideramos então $r \geq 2$ e fixamos um $j \in\{s+1, \ldots, n\}$. Vamos identificar os elementos $\sigma^{\prime \prime}-\sigma_{j} e_{j} \operatorname{com}\left(\sigma_{s+1}, \ldots, \sigma_{j-1}, \sigma_{j+1}, \ldots, \sigma_{n}\right)$.

Definimos o conjunto

$$
Q_{j}=\left\{\left(t^{\prime \prime}, \sigma^{\prime \prime}-\sigma_{j} e_{j}\right) \in[0,2 \pi]^{r} \times[0,2 \pi]^{r-1} ; q\left(t^{\prime \prime}-\sigma^{\prime \prime}+\sigma_{j} e_{j}\right)=0\right\} .
$$

Logo, $\left(t^{\prime \prime}, \sigma^{\prime \prime}-\sigma_{j} e_{j}\right) \in Q_{j}$ se, e somente se, $t^{\prime \prime}-\sigma^{\prime \prime}+\sigma_{j} e_{j}=2 \pi \nu \in[-2 \pi, 2 \pi]^{r}$, com $\nu \in \mathbb{Z}^{r}$.

Considere a função $\psi_{j}:[0,2 \pi]^{n} \times[0,2 \pi]^{r-1} \rightarrow \mathbb{R}$ definida por $\psi_{j}\left(t^{\prime}, t^{\prime \prime}, \sigma^{\prime \prime}-\sigma_{j} e_{j}\right)=B\left(t^{\prime}, t^{\prime \prime}\right)-B\left(0, t^{\prime \prime}-\sigma^{\prime \prime}+\sigma_{j} e_{j}\right)-K q\left(t^{\prime \prime}-\sigma^{\prime \prime}+\sigma_{j} e_{j}\right)-M+\lambda$.

Portanto,

$$
\begin{aligned}
\psi_{j}\left(t^{\prime}, t^{\prime \prime}, \sigma^{\prime \prime}-\sigma_{j} e_{j}\right) & =B\left(t^{\prime}, 2 \pi \nu+\sigma^{\prime \prime}-\sigma_{j} e_{j}\right)-B(0,2 \pi \nu)-M+\lambda \\
& =B\left(t^{\prime}, \sigma_{s+1}, \ldots, \sigma_{j-1}, 0, \sigma_{j+1}, \ldots, \sigma_{n}\right)-M+\lambda \\
& <\frac{\widetilde{M}-M}{2}, \quad \forall\left(t^{\prime}, t^{\prime \prime}, \sigma^{\prime \prime}-\sigma_{j} e_{j}\right) \in[0,2 \pi]^{s} \times Q_{j} .
\end{aligned}
$$


Como $q$ é contínua, o conjunto $Q_{j}$ é fechado (mais que isso, é compacto), logo existe um aberto $U \supset[0,2 \pi]^{s} \times Q_{j}$ tal que

$$
\psi_{j}\left(t^{\prime}, t^{\prime \prime}, \sigma^{\prime \prime}-\sigma_{j} e_{j}\right)<\frac{\widetilde{M}-M}{2}, \quad \forall\left(t^{\prime}, t^{\prime \prime}, \sigma^{\prime \prime}-\sigma_{j} e_{j}\right) \in U .
$$

Considere agora a função $\tilde{q}=q \circ \theta$ sendo $\theta: \mathbb{R}^{n+(r-1)} \rightarrow \mathbb{R}^{r}$ definida por

$$
\theta\left(t^{\prime}, t^{\prime \prime}, \sigma^{\prime \prime}-\sigma_{j} e_{j}\right)=t^{\prime \prime}-\sigma^{\prime \prime}+\sigma_{j} e_{j}=\left(t_{s+1}-\sigma_{s+1}, \ldots, t_{j}, \ldots, t_{n}-\sigma_{n}\right) .
$$

Como $q \geq 0$, temos $\tilde{q} \geq 0$. Afirmamos que a função $\tilde{q}$ não se anula no compacto $\mathcal{K}=\left([0,2 \pi]^{n} \times[0,2 \pi]^{r-1}\right) \backslash U$. De fato, se existisse $\left(t^{\prime}, t^{\prime \prime}, \sigma^{\prime \prime}-\sigma_{j} e_{j}\right) \in \mathcal{K}$ tal que $0=\tilde{q}\left(t^{\prime}, t^{\prime \prime}, \sigma^{\prime \prime}-\sigma_{j} e_{j}\right)=q\left(t^{\prime \prime}-\sigma^{\prime \prime}+\sigma_{j} e_{j}\right)$, teríamos $\left(t^{\prime \prime}, \sigma^{\prime \prime}-\sigma_{j} e_{j}\right) \in Q_{j}$ e portanto $\left(t^{\prime}, t^{\prime \prime}, \sigma^{\prime \prime}-\sigma_{j} e_{j}\right) \in[0,2 \pi]^{s} \times Q_{j}$, o que é um absurdo. Como consequência

$$
0<\mu \doteq \min \left\{\tilde{q}\left(t^{\prime}, t^{\prime \prime}, \sigma^{\prime \prime}-\sigma_{j} e_{j}\right) ; \quad\left(t^{\prime}, t^{\prime \prime}, \sigma^{\prime \prime}-\sigma_{j} e_{j}\right) \in \mathcal{K}\right\}
$$

Portanto, considerando o mesmo $K$ escolhido na proposição anterior obtemos

$$
\begin{aligned}
\psi_{j}\left(t^{\prime}, t^{\prime \prime}, \sigma^{\prime \prime}-\sigma_{j} e_{j}\right) & \leq-B\left(0, t^{\prime \prime}-\sigma^{\prime \prime}+\sigma_{j} e_{j}\right)-K q\left(t^{\prime \prime}-\sigma^{\prime \prime}+\sigma_{j} e_{j}\right)+\lambda \\
& \leq-B\left(0, t^{\prime \prime}-\sigma^{\prime \prime}+\sigma_{j} e_{j}\right)-K \tilde{q}\left(t^{\prime \prime}-\sigma^{\prime \prime}+\sigma_{j} e_{j}\right)+\lambda \\
& \leq-\eta-K \mu+\lambda \\
& <\frac{\widetilde{M}-M}{2}, \quad \forall\left(t^{\prime}, t^{\prime \prime}, \sigma^{\prime \prime}-\sigma_{j} e_{j}\right) \in \mathcal{K},
\end{aligned}
$$

sendo $\eta=\min \left\{B(t) ; t \in[-2 \pi, 2 \pi]^{n}\right\}$. Concluímos assim que

$$
\psi_{j}\left(t^{\prime}, t^{\prime \prime}, \sigma^{\prime \prime}-\sigma_{j} e_{j}\right)<\frac{\widetilde{M}-M}{2}, \quad \forall\left(t^{\prime}, t^{\prime \prime}, \sigma^{\prime \prime}-\sigma_{j} e_{j}\right) \in[0,2 \pi]^{n} \times[0,2 \pi]^{r-1} .
$$

Essa desigualdade juntamente com (3.9) resultam na seguinte estimativa

$$
\left|\hat{f}_{j}(t, \xi)\right| \leq C e^{-\frac{M-\widetilde{M}}{2}}, \quad \forall t \in[0,2 \pi]^{n}, \forall \xi \in \mathbb{N},
$$

na qual a constante $C>0$ não depende de $t$ e $\xi$ (veja o Lema 2.6 na Seção 2.1). Valem também estimativas semelhantes para as derivadas $\partial^{\alpha} \hat{f}_{j}(\cdot, \xi), \alpha \in \mathbb{Z}_{+}^{n}$.

Proposição 3.4. Não existe distribuição $u \in \mathcal{D}^{\prime}\left(\mathbb{T}^{n+1}\right)$ cujos coeficientes parciais de Fourier na variável $x$ sejam os dados em (3.7).

Demonstração. Sejam $t^{*}=\left(t_{*}^{\prime}, t_{*}^{\prime \prime}\right) \in[0,2 \pi]^{n}$ tal que $B\left(t^{*}\right)=M$ e $0<\delta_{0}<\delta$ tal que $|B(t)|<\lambda / 2$ para todo $|t|<\delta_{0}$. Escrevemos $\hat{u}\left(t^{*}, \xi\right)=d_{\xi}\left(I_{\xi}+J_{\xi}\right)$ sendo

$$
I_{\xi}=\int_{\left|t_{*}^{\prime \prime}-\sigma^{\prime \prime}\right|<\delta_{0}} e^{-\xi\left(B\left(0, t_{*}^{\prime \prime}-\sigma^{\prime \prime}\right)+K\left|t_{*}^{\prime \prime}-\sigma^{\prime \prime}\right|^{2}-\lambda\right)} d \sigma^{\prime \prime},
$$


e

$$
J_{\xi}=\int_{\left|t_{*}^{\prime \prime}-\sigma^{\prime \prime}\right| \geq \delta_{0}} e^{-\xi\left(B\left(0, t_{*}^{\prime \prime}-\sigma^{\prime \prime}\right)+K q\left(t_{*}^{\prime \prime}-\sigma^{\prime \prime}\right)-\lambda\right)} d \sigma^{\prime \prime} .
$$

Como $\left|\left(0, t_{*}^{\prime \prime}-\sigma^{\prime \prime}\right)\right|=\left|t_{*}^{\prime \prime}-\sigma^{\prime \prime}\right|<\delta_{0}$ temos $B\left(0, t_{*}^{\prime \prime}-\sigma^{\prime \prime}\right) \leq \lambda / 2$, o que implica a desigualdade

$$
I_{\xi} \geq e^{\xi \lambda / 2} \int_{\left|t_{*}^{\prime \prime}-\sigma^{\prime \prime}\right|<\delta_{0}} e^{-\xi K\left|t_{*}^{\prime \prime}-\sigma^{\prime \prime}\right|^{2}} d \sigma^{\prime \prime} .
$$

Fazendo a mudança de variáveis $\tau=\sqrt{\xi K}\left(t_{*}^{\prime \prime}-\sigma^{\prime \prime}\right)$ na integral anterior obtemos

$$
I_{\xi} \geq \frac{e^{\xi \lambda / 2}}{(\xi K)^{r / 2}} \int_{|\tau|<\delta_{0} \sqrt{\xi K}} e^{-|\tau|^{2}} d \tau
$$

A sequência de números reais positivos $\mu_{\xi}=\int_{|\tau|<\delta_{0} \sqrt{\xi K}} e^{-|\tau|^{2}} d \tau$ é crescente.

Pelo Lema 2.6 (da Seção 2.1) existe uma constante $C>0$ tal que $C^{-1} \leq\left|d_{\xi}\right| \leq C$, para todo $\xi \geq 1$. Tomamos agora $\xi_{0} \in \mathbb{N}$ tal que

$$
e^{\xi \lambda / 4} \geq \widetilde{C} \xi^{r / 2}, \quad \forall \xi \geq \xi_{0}
$$

sendo $\widetilde{C}=\frac{C K^{r / 2}}{\mu_{1}}$.

Multiplicando a desigualdade acima por $e^{\xi \lambda / 4}$ obtemos

$$
\frac{\mu_{1}}{C K^{r / 2}} \frac{e^{\xi \lambda / 2}}{\xi^{r / 2}} \geq e^{\xi \lambda / 4}, \quad \forall \xi \geq \xi_{0} .
$$

Portanto, segue que

$$
\left|\hat{u}\left(t^{*}, \xi\right)\right| \geq \frac{1}{C}\left(I_{\xi}+J_{\xi}\right) \geq \frac{1}{C} I_{\xi} \geq \frac{\mu_{\xi}}{C K^{r / 2}} \frac{e^{\xi \lambda / 2}}{\xi^{r / 2}} \geq e^{\xi \lambda / 4}, \quad \forall \xi \geq \xi_{0} .
$$

donde concluímos que a sequência $\hat{u}(\cdot, \xi)$ não é uma sequência de coeficientes parciais de Fourier de nenhuma distribuição $u \in \mathcal{D}^{\prime}\left(\mathbb{T}^{n+1}\right)$.

\subsection{Um resultado sobre subníveis e superníveis}

Sejam $1 \leq m \leq n$ e $b=\sum_{j=1}^{n} b_{j} d t_{j} \in \bigwedge^{1} C^{\infty}\left(\mathbb{T}^{n} ; \mathbb{R}\right)$ uma 1-forma fechada com períodos $b_{10}, \ldots, b_{n 0}$ racionalmente independentes tais que:

$$
\begin{cases}b_{j}(t)=b_{j}\left(t_{1}, \ldots, t_{m}\right) & j=1, \ldots, m \\ b_{j}(t)=b_{j}\left(t_{m+1}, \ldots, t_{n}\right) & j=m+1, \ldots, n\end{cases}
$$


Seja $B: \mathbb{R}^{n} \rightarrow \mathbb{R}$ uma primitiva global do pull-back $\Pi^{*} b$ através do recobrimento universal $\Pi: \mathbb{R}^{n} \rightarrow \mathbb{T}^{n}$ que nesse caso coincide com o recobrimento minimal segundo a Definição 1.3 do primeiro capítulo. Como os períodos $b_{10}, \ldots, b_{n 0}$ são racionalmente independentes, $B$ recebe o nome de função pseudoperiódica. Essa nomenclatura é devida a Arnold (veja $\mid \mathrm{A}\rfloor$ ).

Vamos denotar por $t=\left(t^{\prime}, t^{\prime \prime}\right)$ as coordenadas em $\mathbb{T}^{n}$ sendo $t^{\prime}=\left(t_{1}, \ldots, t_{m}\right)$ e $t^{\prime \prime}=\left(t_{m+1}, \ldots, t_{n}\right)$. Analogamente, $b_{0}=\left(b_{0}^{\prime}, b_{0}^{\prime \prime}\right)$ sendo $b_{0}^{\prime}=\left(b_{10}, \ldots, b_{m 0}\right)$ e $b_{0}^{\prime \prime}=$ $\left(b_{(m+1) 0}, \ldots, b_{n 0}\right)$.

Existem funções suaves e $2 \pi$-periódicas $P_{1}: \mathbb{R}^{m} \rightarrow \mathbb{R}$ e $P_{2}: \mathbb{R}^{n-m} \rightarrow \mathbb{R}$ tal que a função $B$ pode ser escrita na seguinte forma:

$$
B(t)=B_{1}\left(t^{\prime}\right)+B_{2}\left(t^{\prime \prime}\right),
$$

na qual $B_{1}\left(t^{\prime}\right)=b_{0}^{\prime} \cdot t^{\prime}+P_{1}\left(t^{\prime}\right)$ e $B_{2}\left(t^{\prime \prime}\right)=b_{0}^{\prime \prime} \cdot t^{\prime \prime}+P_{2}\left(t^{\prime \prime}\right)$.

Considere as seguintes notações para os subníveis e superníveis das funções $B, B_{1}$ e $B_{2}$,

$$
\Omega_{s}=\left\{t \in \mathbb{R}^{n}, B(t)<s\right\} \quad \text { e } \quad \Omega^{s}=\left\{t \in \mathbb{R}^{n}, B(t)>s\right\}, \quad s \in \mathbb{R}
$$

e

$$
\Omega_{j, s}=\left\{\tau, B_{j}(\tau)<s\right\} \quad \text { e } \quad \Omega_{j}^{s}=\left\{\tau, B_{j}(\tau)>s\right\}, \quad j=1,2, \quad s \in \mathbb{R} .
$$

Na proposição a seguir, usaremos o seguinte resultado do trabalho [A]:

Se $B$ é uma função pseudoperiódica suave, então cada subnível de $B$ possui uma única componente conexa não limitada.

Proposição 3.5. Sejam b a 1-forma definida em (3.10) com períodos racionalmente independentes e $B=B_{1}+B_{2}$ uma primitiva global de $\Pi^{*} b$ através do recobrimento universal $\Pi: \mathbb{R}^{n} \rightarrow \mathbb{T}^{n}$. Então, todos os subniveis da função $B=B_{1}+B_{2}$ são conexos em $\mathbb{R}^{n}$ se, e somente se, todos os subniveis da função $B_{1}$ são conexos em $\mathbb{R}^{m}$ ou todos os subníveis da função $B_{2}$ são conexos em $\mathbb{R}^{n-m}$.

Demonstração. Suponha que $B_{1}$ e $B_{2}$ possuem subníveis desconexos. Sejam eles $\Omega_{1, r}$ e $\Omega_{2, s}$, com $r, s \in \mathbb{R}$. Pelo trabalho $|\mathrm{A}|$, os subníveis $\Omega_{1, r}$ e $\Omega_{2, s}$ possuem componentes conexas limitadas as quais denotamos por $\omega_{1} \subset \Omega_{1, r}$ e por $\omega_{2} \subset \Omega_{2, s}$. Assim, valem as seguintes propriedades:

- Se $\partial \omega_{1}$ denota a fronteira de $\omega_{1}$ temos $\partial \omega_{1} \subset\left\{t^{\prime} \in \mathbb{R}^{m}, B_{1}\left(t^{\prime}\right)=r\right\}$, assim como $\partial \omega_{2} \subset\left\{t^{\prime \prime} \in \mathbb{R}^{m-n}, B_{2}\left(t^{\prime \prime}\right)=s\right\} ;$

- Se $\mu_{1}$ denota o mínimo de $B_{1}$ sobre $\bar{\omega}_{1}$ e $\mu_{2}$ o mínimo de $B_{2}$ sobre $\bar{\omega}_{2}$ então $r>\mu_{1}$ e $s>\mu_{2}$. 
Considere o conjunto aberto $U=\omega_{1} \times \omega_{2}$. Então, $B(t)=B_{1}\left(t^{\prime}\right)+B_{2}\left(t^{\prime \prime}\right) \geq \mu_{1}+\mu_{2} \doteq$ $\mu$ para todo $t \in U$. Além disso, se $t=\left(t^{\prime}, t^{\prime \prime}\right) \in \partial U=\partial \omega_{1} \times \bar{\omega}_{2} \cup \bar{\omega}_{1} \times \partial \omega_{2}$, então $B(t)=B_{1}\left(t^{\prime}\right)+B_{2}\left(t^{\prime \prime}\right)>\mu_{1}+\mu_{2}=\mu$, $\log \alpha \mu<\tilde{\mu} \doteq \min \{B(t) ; t \in \partial U\}$. Escolhemos agora $\mu<\delta<\tilde{\mu}$.

Afirmamos que o subnível $\Omega_{\delta}=\left\{t \in \mathbb{R}^{n}, B(t)<\delta\right\}$ é desconexo. De fato, escrevemos

$$
\Omega_{\delta}=\left(U \cap \Omega_{\delta}\right) \cup\left(\Omega_{\delta} \backslash U\right),
$$

e tomamos $t_{*}=\left(t_{*}^{\prime}, t_{*}^{\prime \prime}\right) \in U$ tal que $B_{1}\left(t_{*}^{\prime}\right)=\mu_{1}$ e $B_{2}\left(t_{*}^{\prime \prime}\right)=\mu_{2}$, assim $B\left(t_{*}\right)=\mu<\delta$ e, portanto, $t_{*} \in \Omega_{\delta}$ donde concluímos que $U \cap \Omega_{\delta} \neq \emptyset$. Temos também $\Omega_{\delta} \backslash U \neq \emptyset$, caso contrário $\Omega_{\delta} \subset U$ o que é um absurdo pois $\Omega_{\delta}$ é não limitado.

Como $U \cap \Omega_{\delta}$ é aberto em $\mathbb{R}^{n}$, ao mostrarmos que $\Omega_{\delta} \backslash U$ também é um aberto em $\mathbb{R}^{n}$ concluiremos nossa afirmação. Seja então $t \in \Omega_{\delta} \backslash U$. Existe uma vizinhança $V$ de $t$ em $\mathbb{R}^{n}$ tal que $V \cap U=\emptyset$, caso contrário teríamos $V_{t} \cap U \neq \emptyset$ para toda vizinhança $V_{t}$ de $t$ e dessa forma $t \in \partial U$, o que implicaria $B(t) \geq \tilde{\mu}>\delta$, o que é um absurdo uma vez que $t \in \Omega_{\delta}$.

Além disso, pela continuidade de $B$, existe uma vizinhança $W$ de $t$ tal que $W \subset \Omega_{\delta}$. Portanto, $V \cap W \neq \emptyset$ é um aberto de $\mathbb{R}^{n}$ tal que $t \in V \cap W \subset \Omega_{\delta} \backslash U$.

Por outro lado, suponha que $B_{1}$ possui apenas subníveis conexos em $\mathbb{R}^{m}$. Dado $r \in \mathbb{R}$, vamos provar que o subnível $\Omega_{r}=\left\{t \in \mathbb{R}^{n}, B(t)<r\right\}$ é conexo por caminhos. De fato, sejam $p$ e $q \in \Omega_{r}$. Vamos usar a notação $p=\left(p^{\prime}, p^{\prime \prime}\right)$ sendo $p^{\prime}=\left(p_{1}, \ldots, p_{m}\right)$ e $p^{\prime \prime}=\left(p_{m+1}, \ldots, p_{n}\right)$ e analogamente $q=\left(q^{\prime}, q^{\prime \prime}\right)$. Podemos supor que $B_{2}\left(p^{\prime \prime}\right) \leq B_{2}\left(q^{\prime \prime}\right)$, $\operatorname{assim} B_{1}\left(q^{\prime}\right)+B_{2}\left(p^{\prime \prime}\right) \leq B_{1}\left(q^{\prime}\right)+B_{2}\left(q^{\prime \prime}\right)<r$ o que implica $\left(q^{\prime}, p^{\prime \prime}\right) \in \Omega_{r}$.

1을 Caminho: Se $r_{0} \doteq r-B_{2}\left(p^{\prime \prime}\right)$ obtemos $p^{\prime}, q^{\prime} \in \Omega_{1, r_{0}}$. Como $\Omega_{1, r_{0}}$ é aberto e conexo, existe um caminho $\sigma_{1}:[0,1] \rightarrow \mathbb{R}^{m}$ contido em $\Omega_{1, r_{0}}$ tal que $\sigma_{1}(0)=p^{\prime}$ e $\sigma_{1}(1)=q^{\prime}$. Assim, se $\tilde{\sigma}_{1} \doteq\left(\sigma_{1}, p^{\prime \prime}\right)$ segue que

$$
B\left(\sigma_{1}(s), p^{\prime \prime}\right)=B_{1}\left(\sigma_{1}(s)\right)+B_{2}\left(p^{\prime \prime}\right)<r_{0}+B_{2}\left(p^{\prime \prime}\right)=r, \quad \forall s \in[0,1]
$$

ou seja, $\tilde{\sigma}_{1}$ é um caminho em $\mathbb{R}^{n}$ contido no subnível $\Omega_{r}$ que conecta os pontos $p=$ $\left(p^{\prime}, p^{\prime \prime}\right)$ e $\left(q^{\prime}, p^{\prime \prime}\right)$.

$2^{o}$ Caminho: Definimos agora o caminho $\gamma:[0,1] \rightarrow \mathbb{R}^{n-m}$ por

$$
\gamma(s)=\left(s q_{m+1}+(1-s) p_{m+1}, \ldots, s q_{n}+(1-s) p_{n}\right)
$$

Note que $\gamma(0)=p^{\prime \prime}$ e $\gamma(1)=q^{\prime \prime}$. Se $B\left(q^{\prime}, \gamma(s)\right)<r$ para todo $s \in[0,1]$, escolhemos $\sigma_{2}=\left(q^{\prime}, \gamma\right)$ e a demonstração estará concluída tomando o caminho justaposto $\sigma=$ $\tilde{\sigma}_{1}+\sigma_{2}$. Caso isso não ocorra continuamos a construção. 
Escolhemos $k \in \mathbb{N}$ suficientemente grande tal que

$$
B\left(q^{\prime}, \gamma(s)\right)<r+k\left(-2 \pi\left(b_{10}+\cdots+b_{m 0}\right)\right), \quad \forall s \in[0,1] .
$$

Seja $K=(k, \ldots, k) \in \mathbb{Z}^{m}$. Então,

$$
\begin{aligned}
B\left(q^{\prime}+2 \pi K, p^{\prime \prime}\right) & =B_{1}\left(q_{1}+2 \pi k, \ldots, q_{m}+2 \pi k\right)+B_{2}\left(p^{\prime \prime}\right) \\
& =B_{1}\left(q^{\prime}\right)+2 \pi k\left(b_{10}+\cdots+b_{m 0}\right)+B_{2}\left(p^{\prime \prime}\right) \\
& =B\left(q^{\prime}, p^{\prime \prime}\right)+2 \pi k\left(b_{10}+\cdots+b_{m 0}\right) \\
& =B\left(q^{\prime}, \gamma(0)\right)+2 \pi k\left(b_{10}+\cdots+b_{m 0}\right)<r .
\end{aligned}
$$

Portanto, $\left(q^{\prime}+2 \pi K, p^{\prime \prime}\right) \in \Omega_{r}$ e $q^{\prime}+2 \pi K \in \Omega_{1, r_{0}}$.

Como $q^{\prime}+2 \pi K$ e $q^{\prime} \in \Omega_{1, r_{0}}$, existe um caminho $\sigma_{2}$ em $\mathbb{R}^{m}$ contido no subnível $\Omega_{1, r_{0}}$. Agora, analogamente à construção feita para o primeiro caminho $\tilde{\sigma}_{1}$, obtemos um caminho $\tilde{\sigma}_{2}$ contido em $\Omega_{r}$ conectando o ponto $\left(q^{\prime}, p^{\prime \prime}\right)$ ao ponto $\left(q^{\prime}+2 \pi K, p^{\prime \prime}\right)$.

$3^{\text {o }}$ Caminho: Considere $\sigma_{3}:[0,1] \rightarrow \mathbb{R}^{n}$ definido por $\sigma_{3}=\left(q^{\prime}+2 \pi K, \gamma\right)$, sendo $\gamma$ como anteriormente. Então

$$
B\left(q^{\prime}+2 \pi K, \gamma(s)\right)=B\left(q^{\prime}, \gamma(s)\right)+2 \pi k\left(b_{10}+\cdots+b_{m 0}\right)<r, \quad \forall s \in[0,1] .
$$

Portanto, $\sigma_{3}$ é um caminho em $\mathbb{R}^{n}$ contido no subnível $\Omega_{r}$ conectando os pontos $\left(q^{\prime}+2 \pi K, p^{\prime \prime}\right)$ e $\left(q^{\prime}+2 \pi K, q^{\prime \prime}\right)$.

$4^{o}$ Caminho: Como $B_{1}\left(q^{\prime}\right)<r-B_{2}\left(q^{\prime \prime}\right) \doteq r_{1}$ temos $B_{1}\left(q^{\prime}+2 \pi K\right)<B_{1}\left(q^{\prime}\right)<r_{1}$. Portanto, existe um caminho $\sigma_{4}:[0,1] \rightarrow \mathbb{R}^{m}$ contido no subnível $\Omega_{1, r_{1}}$ tal que $\sigma_{4}(0)=$ $q^{\prime}+2 \pi K$ e $\sigma_{4}(1)=q^{\prime}$. Definimos $\tilde{\sigma}_{4}=\left(\sigma_{4}, q^{\prime \prime}\right)$. Então,

$$
B\left(\sigma_{4}(s), q^{\prime \prime}\right)<r_{1}+B_{2}\left(q^{\prime \prime}\right)=r, \quad \forall s \in[0,1]
$$

assim, $\tilde{\sigma}_{4}$ é um caminho em $\mathbb{R}^{n}$ contido no subnível $\Omega_{r}$ conectando $\left(q^{\prime}+2 \pi K, q^{\prime \prime}\right)$ e $q=\left(q^{\prime}, q^{\prime \prime}\right)$.

Finalmente, o caminho justaposto formado pelos 4 caminhos anteriores está contido em $\Omega_{r}$ e conecta os pontos $p$ e $q$.

Uma demonstração semelhante à feita para a proposição anterior garante que o mesmo ocorre como os superníveis, ou seja,

Proposição 3.6. Sejam b a 1-forma definida em (3.10) com períodos racionalmente independentes e $B=B_{1}+B_{2}$ uma primitiva global de $\Pi^{*} b$ através do recobrimento universal $\Pi: \mathbb{R}^{n} \rightarrow \mathbb{T}^{n}$. Então, todos os superniveis da função $B=B_{1}+B_{2}$ são conexos em $\mathbb{R}^{n}$ se, e somente se, todos os superniveis da função $B_{1}$ são conexos em $\mathbb{R}^{m}$ ou todos os superníveis da função $B_{2}$ são conexos em $\mathbb{R}^{n-m}$. 
Observação: No caso em que a 1 -forma $b$ possui períodos racionalmente dependentes podem não valer os resultados anteriores no recobrimento minimal de $\mathbb{T}^{n}$ com relação a $b$. De fato, seja $B: \mathbb{T}^{1} \times \mathbb{R} \rightarrow \mathbb{R}$ definida por

$$
B\left(t_{1}, t_{2}\right)=-\cos t_{1}+\left(\operatorname{sen} t_{2}-\frac{\sqrt{2}}{2} t_{2}\right) .
$$

Logo, todos os subníveis e superníveis da função $B_{1}\left(t_{1}\right)=-\cos t_{1}$ são conexos em $\mathbb{S}^{1} \simeq \mathbb{T}^{1}$. Por outro lado, pelo Lema 2.2 , a função $B$ possui subníveis e superníveis desconexos em $\mathbb{T}^{1} \times \mathbb{R}$ uma vez que $b_{1}\left(t_{1}\right)=\operatorname{sen} t_{1}$ e $b_{2}\left(t_{2}\right)=\cos t_{2}-\sqrt{2} / 2$ mudam de sinal.

\subsection{Sistema parcialmente acoplado - um caso}

Apresentamos nesta seção um sistema com um acoplamento mais forte. Obtemos condições necessárias e suficientes para a resolubilidade global desse sistema. Para isso, foram usados os resultados das duas seções anteriores e do trabalho [BK].

Considere o operador

$$
\mathbb{L}=d_{t}+i b(t) \wedge \frac{\partial}{\partial x}
$$

sendo $b \in \bigwedge^{1} C^{\infty}\left(\mathbb{T}^{2 n} ; \mathbb{R}\right)$ uma 1-forma fechada dada como segue:

$$
b(t)=\sum_{j=1}^{n} b_{2 j-1}\left(t_{2 j-1}, t_{2 j}\right) d t_{2 j-1}+\sum_{j=1}^{n} b_{2 j}\left(t_{2 j-1}, t_{2 j}\right) d t_{2 j} .
$$

Além disso, vamos supor que os períodos $b_{10}, \ldots, b_{2 n 0}$ sejam racionalmente independentes, isto é, o posto do grupo de períodos da 1-forma $b$ é igual a $2 n$.

Seja $B: \mathbb{R}^{2 n} \rightarrow \mathbb{R}$ uma primitiva global do pull-back $\Pi^{*} b$ através do recobrimento universal $\Pi: \mathbb{R}^{2 n} \rightarrow \mathbb{T}^{2 n}$. Então, a função $B$ pode ser escrita na seguinte forma

$$
B(t)=B_{12}\left(t_{1}, t_{2}\right)+B_{34}\left(t_{3}, t_{4}\right)+\cdots+B_{(2 n-1) 2 n}\left(t_{2 n-1}, t_{2 n}\right) .
$$

Na demonstração da Proposição 3.8, apresentada a seguir, usamos resultados do trabalho $[\mathrm{BK}]$ que tratou da resolubilidade global do operador $\mathbb{L}$ definido em (3.11) no caso bidimensional; mais precisamente, o resultado provado foi o seguinte:

Teorema 3.7 (Bergamasco e Kirilov). Sejam $b \in \bigwedge^{1} C^{\infty}\left(\mathbb{T}^{2} ; \mathbb{R}\right)$ uma 1-forma fechada com periodos $b_{10}$ e $b_{20}$ racionalmente independentes e $B$ uma primitiva global do pullback $\Pi^{*} b$ através do recobrimento universal $\Pi: \mathbb{R}^{2} \rightarrow \mathbb{T}^{2}$. Então, o operador $\mathbb{L}$ definido em (3.11) é globalmente resolúvel em $\mathbb{T}^{3}$ se, e somente se, os subníveis $\Omega_{s}=\{t \in$ $\left.\mathbb{R}^{2}, B(t)<s\right\}$ e superníveis $\Omega^{s}=\left\{t \in \mathbb{R}^{2}, B(t)>s\right\}$ são conexos para todo $s \in \mathbb{R}$. 
Proposição 3.8. Sejam b a 1-forma definida em (3.12) com periodos racionalmente independentes e $B$ dada em (3.13) uma primitiva global do pull-back $\Pi^{*} b$ através do recobrimento universal $\Pi: \mathbb{R}^{2 n} \rightarrow \mathbb{T}^{2 n}$. Então, o operador $\mathbb{L}$ definido em (3.11) é globalmente resolúvel em $\mathbb{T}^{2 n+1}$ se, e somente se, os subniveis $\Omega_{s}=\left\{t \in \mathbb{R}^{2 n}, B(t)<s\right\}$ e superniveis $\Omega^{s}=\left\{t \in \mathbb{R}^{2 n}, B(t)>s\right\}$ são conexos para todo $s \in \mathbb{R}$.

Demonstração. Suponha que existe um subnível desconexo da função $B$ em $\mathbb{R}^{2 n}$. Então, pela Proposição 3.5 cada função $B_{(2 j-1), 2 j}, j=1, \ldots, n$, possui um subnível desconexo em $\mathbb{R}^{2}$. Caso exista um supernível desconexo de $B$, então cada função $B_{(2 j-1) 2 j}, j=$ $1, \ldots, n$, também possui um supernível desconexo em $\mathbb{R}^{2}$.

Portanto, pelo trabalho $[\mathrm{BK}]$, existem difeomorfismos $\phi_{1}, \ldots, \phi_{n}$ em $\mathbb{T}^{2 n}$ tais que cada $\phi_{j}$ age somente nas coordenadas $t_{2 j-1}, t_{2 j}$ produzindo novas coordenadas de forma que o máximo da função $B_{(2 j-1) 2 j}$ sobre o cubo $[0,2 \pi]_{t_{2 j-1}, t_{2 j}}^{2}$ não ocorre na fronteira (ver Seção 5 de $[\mathrm{BK}\rfloor)$. Fazendo a composição desses difeomorfismos obtemos um sistema de coordenadas em $\mathbb{T}^{2 n}$ de forma que o máximo da função $B$ sobre o cubo $[0,2 \pi]^{2 n}$ não ocorre na fronteira. Então, pelo Teorema 3.1 concluímos que $\mathbb{L}$ não é globalmente resolúvel em $\mathbb{R}^{2 n+1}$.

Passamos agora à demonstração da suficiência da proposição. Primeiramente obtemos caminhos de integração que serão convenientes para a construção de uma solução da equação $\mathbb{L} u=f$.

Lema 3.9. Se todos os subníveis de $B$ são conexos em $\mathbb{R}^{2 n}$, então existe uma constante $C>0$ tal que para cada $t \in[0,2 \pi]^{2 n}$ e cada $\xi \in \mathbb{Z}$ existe um caminho poligonal $\gamma=\gamma(t, \xi)$ conectando os pontos $t$ e $t+(2 \pi, 0, \ldots, 0)$ com as seguintes propriedades:

1. $B(\tau) \leq B(t)+\frac{1}{1+|\xi|}$ para todo $\tau \in \gamma(t, \xi)$;

2. O comprimento $|\gamma(t, \xi)|<C(1+|\xi|)$.

Demonstração. Como todos os subníveis de $B$ são conexos em $\mathbb{R}^{2 n}$, então pela Proposição 3.5 existe uma função $B_{(2 j-1) 2 j}, j=1, \ldots, n$, que possui todos os subníveis conexos em $\mathbb{R}^{2}$. Reordenando as coordenadas podemos assumir que $j=1$, ou seja, todos os subníveis da função $B_{12}$ são conexos em $\mathbb{R}^{2}$.

Vamos mostrar agora que existe uma faixa $S=\left\{\left(s_{1}, s_{2}\right) \in \mathbb{R}^{2} ;\left|b_{10} s_{1}+b_{20} s_{2}\right| \leq \nu\right\}$ que contém todos os conjuntos de nível $B_{12}^{-1}\left\{B_{12}(\tau)\right\}, \tau \in[-2 \pi, 4 \pi] \times[0,2 \pi]$. Com efeito, considere a função $2 \pi$-periódica $P\left(s_{1}, s_{2}\right)=B_{12}\left(s_{1}, s_{2}\right)-b_{10} s_{1}-b_{20} s_{2}$, dessa forma, se $s=\left(s_{1}, s_{2}\right) \in B_{12}^{-1}\left\{B_{12}(\tau)\right\}$ então

$$
\begin{array}{r}
0=B_{12}(\tau)-B_{12}(s) \leq 2\|P\|_{\infty}+b_{10}\left(\tau_{1}-s_{1}\right)+b_{20}\left(\tau_{2}-s_{2}\right), \quad \mathrm{e} \\
0=B_{12}(s)-B_{12}(\tau) \leq 2\|P\|_{\infty}+b_{10}\left(s_{1}-\tau_{1}\right)+b_{20}\left(s_{2}-\tau_{2}\right),
\end{array}
$$


$\log 0$

$$
\begin{gathered}
b_{10} s_{1}+b_{20} s_{2} \leq 2\|P\|_{\infty}+b_{10} \tau_{1}+b_{20} \tau_{2} \leq 2\|P\|_{\infty}+4 \pi\left|b_{10}\right|+2 \pi\left|b_{20}\right| \doteq \nu, \quad \mathrm{e} \\
b_{10} s_{1}+b_{20} s_{2} \geq-2\|P\|_{\infty}+b_{10} \tau_{1}+b_{20} \tau_{2} \geq-2\|P\|_{\infty}-\left|b_{10}\right|\left|\tau_{1}\right|-\left|b_{20}\right|\left|\tau_{2}\right| \geq-\nu .
\end{gathered}
$$

Fixemos $t=\left(t_{1}, \ldots, t_{2 n}\right) \in[0,2 \pi]^{2 n}$ e $\xi \in \mathbb{Z}$. Sejam $r=B_{12}\left(t_{1}, t_{2}\right)+1 / 2(1+|\xi|)$ e $\Omega_{1, r}=\left\{\tau \in \mathbb{R}^{2} ; B_{12}(\tau)<r\right\}$. Afirmamos que o conjunto

$$
K_{r}=\Omega_{1, r} \cap([-2 \pi, 4 \pi] \times \mathbb{R}) \cap S
$$

é limitado e conexo por caminhos. Antes de provar a afirmação feita, pelo Teorema 4.30 de $[\mathrm{BK}]$ é possível obter um sistema de coordenadas em $\mathbb{T}^{2 n}$ tal que para cada $k \in \mathbb{Z}$ a função $\tau_{2} \mapsto B_{12}\left(2 \pi k, \tau_{2}\right)$ é decrescente.

Dando continuidade, uma vez que as faixas $[-2 \pi, 4 \pi] \times \mathbb{R}$ e $S$ não são paralelas em $\mathbb{R}^{2}$ então $K_{r}$ é limitado. Sejam agora $p, q \in K_{r}$, então existe um caminho $\sigma$ contido no subnível $\Omega_{1, r}$ conectando $p$ e $q$.

Se $\sigma \cap(-\infty,-2 \pi) \times \mathbb{R} \neq \emptyset$, ou em outras palavras, o caminho $\sigma$ sai pela esquerda da faixa $[-2 \pi, 4 \pi] \times \mathbb{R}$, então tome o primeiro ponto $p_{0} \in \sigma$ e último ponto $q_{0} \in \sigma$ (sobre a linha $-2 \pi \times \mathbb{R}$ ) onde $\sigma$ sai da faixa $[-2 \pi, 4 \pi] \times \mathbb{R}$ pela esquerda. Dessa forma, substituímos o segmento do caminho $\sigma$ do ponto $p_{0}$ ao ponto $q_{0}$, pelo segmento de reta $\left[\left[p_{0}, q_{0}\right]\right] \subset \Omega_{1, r}$. Se o caminho $\sigma$ sai pela direita da faixa $[-2 \pi, 4 \pi] \times \mathbb{R}$ repetimos o mesmo procedimento. Para concluir a demonstração de que $K_{r}$ é conexo por caminhos, note que a reta $b_{10} s_{1}+b_{20} s_{2}=-\nu$ está contida no subnível $\Omega_{1, r}$, dessa forma, se o caminho $\sigma$ sair da faixa $S$ repetimos o procedimento usado anteriormente.

Seja $D_{0}$ a coleção de quadrados em $\mathbb{R}^{2}$ com lado $2 \pi$ e com vértices em $2 \pi \mathbb{Z}^{2}$. Para cada $k \in \mathbb{N}$ denotamos por $D_{k}$ a imagem de $D_{0}$ através da contração $\tau \mapsto 2^{-k} \tau$. Definimos agora os conjuntos

$$
A_{k r}=\left\{Q \in D_{k} ; Q \cap K_{r} \neq \emptyset\right\} \quad \text { e } \quad V_{k r}=\bigcup_{Q \in A_{k r}} Q
$$

Uma vez que $K_{r}$ é limitado e conexo por caminhos então $V_{k r}$ também possui estas propriedades. Como $\left(t_{1}, t_{2}\right)$ e $\left(t_{1}, t_{2}\right)+(2 \pi, 0) \in K_{r}$, então existe um caminho contido em $V_{k r}$ conectando esses pontos. Seja $\gamma_{0}:[0,2 \pi] \mapsto \mathbb{R}^{2}$ esse caminho.

Construiremos um caminho poligonal a partir de $\gamma_{0}$ da seguinte forma: em cada quadrado $Q \in D_{k}$ tal que $Q \cap \gamma_{0} \neq \emptyset$, tome o primeiro e o último ponto de intersecção. Ligando estes pontos obtemos uma poligonal $\gamma_{1}$ contida em $V_{k r}$ conectando $\left(t_{1}, t_{2}\right)$ e $\left(t_{1}, t_{2}\right)+(2 \pi, 0)$ 
Definimos agora o seguinte caminho poligonal em $\mathbb{R}^{2 n}$. Seja $\gamma:[0,2 \pi] \mapsto \mathbb{R}^{2 n}$ definido por $\gamma=\left(\gamma_{1}, t_{3}, t_{4}, \ldots, t_{2 n}\right)$. Vejamos que $\gamma=\gamma(t, \xi)$ possui as propriedades 1 e 2 enunciadas no lema.

Seja $s=\left(s_{1}, s_{2}, t_{3}, t_{4}, \ldots, t_{2 n}\right) \in \gamma(t, \xi)$, assim $\left(s_{1}, s_{2}\right) \in \gamma_{1} \subset V_{k r}$, ou seja, $\left(s_{1}, s_{2}\right)$ pertence a algum quadrado $Q \in D_{k}$ tal que $Q \cap K_{r} \neq \emptyset$. Tome então $\left(s_{1}^{\prime}, s_{2}^{\prime}\right) \in Q \cap K_{r}$. Portanto, $B_{12}\left(s_{1}^{\prime}, s_{2}^{\prime}\right)<r$ e como a diagonal de cada quadrado da coleção $D_{k}$ tem comprimento $(\pi \sqrt{2}) 2^{-(k-1)}$, segue que $\left|\left(s_{1}, s_{2}\right)-\left(s_{1}^{\prime}, s_{2}^{\prime}\right)\right| \leq(\pi \sqrt{2}) 2^{-(k-1)}$.

Vamos agora escolher um $k \in \mathbb{N}$ conveniente: tomamos o único $k \in \mathbb{N}$ que satisfaz

$$
2^{k-1}<4 \pi \sqrt{2}(1+|\xi|)\|b\|_{\infty} \leq 2^{k}
$$

$\log \mathrm{O}$

$$
\frac{1}{4(1+|\xi|)}<\frac{\pi \sqrt{2}\|b\|_{\infty}}{2^{k-1}} \leq \frac{1}{2(1+|\xi|)} .
$$

Sejam $s^{\prime}=\left(s_{1}^{\prime}, s_{2}^{\prime}, t_{3}, t_{4}, \ldots, t_{2 n}\right)$ e $r_{0}=r+B_{34}\left(t_{3}, t_{4}\right)+\cdots+B_{(2 n-1) 2 n}\left(t_{2 n-1}, t_{2 n}\right)$, então

$$
\begin{aligned}
B(s) & \leq\left|B(s)-B\left(s^{\prime}\right)\right|+B\left(s^{\prime}\right) \leq\left|B_{12}\left(s_{1}, s_{2}\right)-B_{12}\left(s_{1}^{\prime}, s_{2}^{\prime}\right)\right|+r_{0} \\
& \leq\|b\|_{\infty}\left|\left(s_{1}, s_{2}\right)-\left(s_{1}^{\prime}, s_{2}^{\prime}\right)\right|+r_{0} \leq\|b\|_{\infty} \frac{\pi \sqrt{2}}{2^{k-1}}+r_{0} \\
& \leq \frac{1}{2(1+|\xi|)}+r_{0}=\frac{1}{2(1+|\xi|)}+\left(\frac{1}{2(1+|\xi|)}+B(t)\right) \\
& =B(t)+\frac{1}{1+|\xi|},
\end{aligned}
$$

o que conclui a demonstração do item 1 .

Além disso, se $N$ é o número de quadrados em $D_{0}$ que intersectam $K_{r}$ então o número de quadrados em $D_{k}$ que intersectam o conjunto $K_{r}$ é no máximo $4^{k} N$. Assim, por (3.14), o comprimento $|\gamma(t, \xi)|$ satisfaz

$$
|\gamma(t, \xi)|=\left|\gamma_{1}(t, \xi)\right| \leq \frac{\pi \sqrt{2}}{2^{k-1}} 4^{k} N=(4 \pi N \sqrt{2}) 2^{k-1}<(4 \pi N \sqrt{2}) 4 \pi \sqrt{2}\|b\|_{\infty}(1+|\xi|),
$$

o que conclui a demonstração do lema.

Lema 3.10. Se todos os superniveis de $B$ são conexos em $\mathbb{R}^{2 n}$, então existe uma constante $C>0$ tal que para cada $t \in[0,2 \pi]^{2 n}$ e cada $\xi \in \mathbb{Z}$ existe um caminho poligonal $\gamma=\gamma(t, \xi)$ conectando os pontos $t$ e $t-(2 \pi, 0, \ldots, 0)$ com as seguintes propriedades:

1. $B(\tau) \geq B(t)-\frac{1}{1+|\xi|}$ para todo $\tau \in \gamma(t, \xi)$ 
2. O comprimento $|\gamma(t, \xi)|<C(1+|\xi|)$.

Demonstração. Basta considerar a função $\widetilde{B}=-B$ e aplicar mesmas ideias do lema anterior.

Observação: Com pequenas correções nos caminhos $\gamma(t, \xi)$ construídos anteriormente, podemos supor que eles são suaves e ainda possuem as propriedades enunciadas nos Lemas 3.9 e 3.10 .

Construção da Solução: seja $f \in \mathbb{E}$. Vamos usar série parcial de Fourier na variável $x$ para construir uma solução suave $u$ da equação $\mathbb{L} u=f$. Substituindo as séries formais

$$
u(t, x)=\sum_{\xi \in \mathbb{Z}} \hat{u}(t, \xi) e^{i \xi x} \quad \text { e } \quad f(t, x)=\sum_{\xi \in \mathbb{Z}} \hat{f}(t, \xi) e^{i \xi x}
$$

na equação $\mathbb{L} u=d_{t} u+i b(t) \wedge \frac{\partial}{\partial x} u=f$, obtemos para cada $\xi \in \mathbb{Z}$

$$
\left(d_{t}-\xi b(t) \wedge\right) \hat{u}(t, \xi)=\hat{f}(t, \xi), \quad t \in \mathbb{R}^{2 n}
$$

$\log$,

$$
d_{t}\left(e^{-\xi B} \hat{u}(\cdot, \xi)\right)=e^{-\xi B} \hat{f}(\cdot, \xi) .
$$

Ou seja, $e^{-\xi B} \hat{f}(\cdot, \xi)$ é uma 1-forma exata em $\mathbb{R}^{2 n}$ e uma solução geral da equação acima $\left(\right.$ em $\left.\mathbb{R}^{2 n}\right)$ é dada por

$$
\hat{u}(t, \xi)=\int_{t_{0}}^{t} e^{-\xi(B(\tau)-B(t))} \hat{f}(\tau, \xi) d \tau+\hat{u}\left(t_{0}, \xi\right) e^{-\xi\left(B\left(t_{0}\right)-B(t)\right)} .
$$

Uma vez que a integral anterior não depende do caminho, escolhemos curvas suaves $\gamma_{ \pm}:[0,2 \pi] \mapsto \mathbb{R}^{2 n}$ tais que $\gamma_{ \pm}(0)=t_{0}$ e $\gamma_{ \pm}(2 \pi)=t_{0} \mp(2 \pi, 0, \ldots, 0)$ cujas projeções no toro $\mathbb{T}^{2 n}$ são curvas não homologas à zero. As curvas $\gamma_{+}$e $\gamma_{-}$serão escolhidas dependendo do sinal de $\xi$. Portanto, quando $\xi \neq 0$ temos

$$
\hat{u}\left(t_{0}, \xi\right) e^{-\xi B\left(t_{0}\right)}=\frac{1}{e^{ \pm 2 \pi \xi b_{10}}-1} \int_{0}^{2 \pi} e^{-\xi B\left(\gamma_{ \pm}(\sigma)\right)} \hat{f}\left(\gamma_{ \pm}(\sigma), \xi\right) \gamma_{ \pm}^{\prime}(\sigma) d \sigma,
$$

e assim obtemos

$$
\hat{u}\left(\gamma_{ \pm}(s), \xi\right)=\int_{0}^{s} g(\sigma, \xi) d \sigma+\frac{1}{e^{ \pm 2 \pi \xi b_{10}}-1} \int_{0}^{2 \pi} g(\sigma, \xi) d \sigma
$$

sendo $g(\sigma, \xi)=e^{-\xi\left[B\left(\gamma_{ \pm}(\sigma)\right)-B\left(\gamma_{ \pm}(s)\right)\right]} \hat{f}\left(\gamma_{ \pm}(\sigma), \xi\right) \gamma_{ \pm}^{\prime}(\sigma)$. Para $\xi=0$ temos

$$
\hat{u}\left(\gamma_{+}(s), 0\right)=\int_{0}^{s} \hat{f}\left(\gamma_{+}(\sigma), 0\right) \gamma_{+}^{\prime}(\sigma) d \sigma .
$$


Por fim, considere $u$ definida pela série

$$
u(t, x)=\sum_{\xi \in \mathbb{Z}} \hat{u}(t, \xi) e^{i \xi x}
$$

na qual os coeficientes $\hat{u}(\cdot, \xi)$ são dados por (3.15) e (3.16). Para $\xi \geq 0$ escolhemos $\gamma_{+}$ a curva obtida no Lema 3.10 e para $\xi<0$ escolhemos $\gamma_{-}$a curva obtida no Lema 3.9. Dessa forma, $u$ é uma função $C^{\infty}$ e é uma solução da equação $\mathbb{L} u=f$. A demonstração desse fato é análoga a feita no caso bidimensional que encontra-se em [BK]. 



\section{Referências Bibliográficas}

[A] V. I. Arnold, Topological and ergodic properties of closed 1-forms with incommensurable periods, Functional Analysis and its Applications, 25 (1991), 81-90.

[B] A. P. Bergamasco, Remarks about global analytic hypoellipticity, Transactions of the American Mathematical Society, 351 (1999), 4113-4126.

[BCH] S. Berhanu, P. Cordaro e J. Hounie, An Introduction to Involutive Structures, Cambridge University Press, New York, 2008.

[BCM] A. P. Bergamasco, P. Cordaro e P. Malagutti, Globally hypoelliptic systems of vector fields, Journal of Functional Analysis, 114 (1993), 267-285.

[BCP1] A. P. Bergamasco, P. Cordaro e G. Petronilho, Global Solvability for certain classes of undetermined of vector fields, Math. Zeitschrift, 223 (1996), 261-274.

[BCP2] A. P. Bergamasco, P. Cordaro e G. Petronilho, Global solvability for a class of complex vector fields on the two-torus, Communications in Partial Differential Equations, 29 (2004), 785-819.

[BK] A. P. Bergamasco e A. Kirilov, Global solvability for a class of overdetermined systems, Journal of Functional Analysis, 252 (2007), 603-629.

[BKNZ] A. P. Bergamasco, A. Kirilov, W. Nunes e S. Zani, On the global solvability for overdetermined systems, Transactions of the American Mathematical Society, to appear.

[BNZ1] A. P. Bergamasco, W. Nunes e S. Zani, Global analytic hypoellipticity and pseudoperiodic functions, Matemática Contemporânea, 18 (2000), 43-57. 
[BNZ2] A. P. Bergamasco, W. Nunes e S. Zani, Global properties for a class of overdetermined systems, Journal of Functional Analysis, 200 (2003), 31-64.

[BP] A. P. Bergamasco e G. Petronilho, Global solvability of a class of involutive systems, Journal of Mathematical Analysis and Applications, 233 (1999), 314-327.

[BZ] A. P. Bergamasco e S. Zani, Prescribing analytic singularities for solutions of a class of vector fields on the torus, Transactions of the American Mathematical Society, 357 (2005), 4159-4174.

[CH] F. Cardoso e J. Hounie, Global solvability of an abstract complex, Proceedings of the American Mathematical Society, 65 (1977), 117-124.

[H] J. Hounie, Globally hypoelliptic and globally solvable first order evolution equations, Transactions of the American Mathematical Society, 252 (1979), 233-248.

[MMST] F. Martinez, C. Moreira, N. Saldanha e E. Tengan, Teoria dos Números, um passeio com primos e outros números familiares pelo mundo inteiro, Projeto Euclides, IMPA, 2010.

[T1] F. Treves, Study of a model in the theory of complexes of pseudodifferential operators, Annals of Mathematics, (2) 104 (1976), 269-324.

[T2] F. Treves, Hypoanalytic Structures (Local Theory), Princeton University Press, Princeton, NJ, 1992. 


\section{Índice Remissivo}

$\mathcal{D}_{\mathcal{A}}^{\prime}\left(\mathbb{T}^{n+1}\right)$ e $\mathcal{D}_{\mathcal{B}}^{\prime}\left(\mathbb{T}^{n+1}\right), 36$

1-forma complexa $c, 1$

Automorfismo, 17, 22, 36

Campos vetoriais, xi, 1, 13

Complexo diferencial, 2

Condições de compatibilidade, 2

Derivada exterior em $\mathbb{T}^{n}, d_{t}, 2$

Estrutura localmente integrável, 2

Fibrado

cotangente, $\mathbb{C} \otimes T^{*}\left(\mathbb{T}^{n+1}\right), 1$

tangente, $\mathbb{C} \otimes T\left(\mathbb{T}^{n+1}\right), 1$

Função

de corte, 25, 41, 52

pseudoperiódica, 57

Grupo

$S L(n ; \mathbb{Z}), 7$

de permutações $\mathcal{S}_{n}, 23$

de períodos da 1 -forma $b, 5$

Número de Liouville, 10

Operador(es)

$\mathbb{L}, 2,13,17,49,60$
$\mathbb{L}_{\mathcal{A}}$ e $\mathbb{L}_{\mathcal{B}}, 36$

Posto de $\operatorname{Per}(b), 5,6,49$

Recobrimento minimal de $\mathbb{T}^{n}, 5$

Resolubilidade

global, xi, 2

local, xi

Sistema involutivo

desacoplado, 13

do tipo tubo, xi

parcialmente acoplado, 60

Subfibrado $T^{\prime}, 1$

Série parcial de Fourier, 2, 17, 34 